\title{
The PHEMU97 catalogue of observations of the mutual phenomena of the Galilean satellites of Jupiter ${ }^{\star}$
}

\author{
J.-E. Arlot ${ }^{1}$, W. Thuillot ${ }^{1}$, C. Ruatti ${ }^{1}$, H. Akasawa ${ }^{2}$, S. Baroni1 ${ }^{12}$, W. Beisker ${ }^{13}$, J. Berthier ${ }^{1}$, C. Blanco ${ }^{3}$, \\ J. Boonstra ${ }^{16}$, J. Bourgeois ${ }^{17}$, H. Bulder ${ }^{18}$, R. Casas $^{19}$, J. G. Castano ${ }^{35}$, F. Colas ${ }^{1}$, D. Collins ${ }^{20}$, J. Cuypers ${ }^{21}$, \\ W. Czech ${ }^{15}$, V. D'Ambrosio ${ }^{3}$, H. Denzau ${ }^{22}$, P. Descamps ${ }^{1}$, A. Dimitrescu ${ }^{8}$, N. Dinakarian ${ }^{34}$, G. Dourneau ${ }^{5}$, \\ N. Emelyanov ${ }^{11}$, J. M. Enriquez ${ }^{23}$, J. M. Fernandez ${ }^{24}$, D. Fernandez-Barba ${ }^{1}$, T. Flatres ${ }^{5}$, S. Foglia ${ }^{14}$, \\ M. Goncalves ${ }^{28}$, K. Guhl ${ }^{26}$, G. Helmer ${ }^{6}$, T. Hirose ${ }^{44}$, T. R. Irsmambetova ${ }^{9}$, B. A. Krobusek ${ }^{39}$, J. Lecacheux ${ }^{7}$, \\ J.-F. Le Campion ${ }^{5}$, M. Lou ${ }^{30}$, A. Mallama ${ }^{42}$, F. Marchis ${ }^{4}$, M. A. S. Navarro ${ }^{30}$, P. Nelson ${ }^{38}$, N. Okura ${ }^{31}$, J. Park ${ }^{38}$, \\ T. Pauwels ${ }^{21}$, S. Pluchino ${ }^{36}$, V. Priban ${ }^{29}$, M. Rapaport ${ }^{5}$, J.-J. Sacré ${ }^{25}$, F. Salvaggio ${ }^{40}$, M. A. Sanchez ${ }^{30}$, \\ F. Sanchez-Bajo ${ }^{27}$, G. Stefanescu ${ }^{8}$, P. Tanga ${ }^{33}$, V. G. Tejfel ${ }^{10}$, J. L. Trisan ${ }^{30}$, E. M. Trunkovsky ${ }^{11}$, J. Van Gestel ${ }^{43}$, \\ G. Vandenbulcke ${ }^{32}$, R. Vasundhara ${ }^{34}$, G. Vass ${ }^{8}$, P. Vingerhoets ${ }^{21}$, D. T. Vu ${ }^{1}$, and R. T. Wilds ${ }^{37}$ \\ (Affiliations can be found after the references)
}

Received 7 October 2005 / Accepted 28 November 2005

ABSTRACT

In 1997 the Sun and the Earth passed through the equatorial plane of Jupiter and therefore through the orbital planes of its main satellites. During this period, mutual eclipses and occultations occurred and were observed. We investigate the precision of the catalogue to produce improved data for the development of dynamical models. Light curves of mutual eclipses and occultations were recorded by the observers of the international campaign PHEMU97 organized by the Institut de Mécanique Céleste, Paris, France. We made 275 observations of 148 mutual events from 42 sites. For each observation, information is given about the telescope, the receiver, the site and the observational conditions. This paper gathers together the data and gives a first estimate of the precision. The catalogue of these rare events represents a collection of improved accurate astrometric data useful for the development of dynamical models.

Key words. eclipses - occultations - astrometry - astronomical data bases: miscellaneous

\section{Introduction}

Observations of mutual events of natural satellites have been performed intensively since 1973 and they have proved to be a very accurate way to obtain astrometric measurements of natural satellites. Many such events involving the Galilean satellites of Jupiter have been observed. In 1994-1996, similar events occurred in the Saturnian system. In 1997, we organized and coordinated an international campaign to observe these rare events. This campaign, named PHEMU97, allowed us to collect 275 lightcurves of 148 mutual events by our international network of 42 sites.

We present our results here. Another paper (Vasundhara et al. 2003) provides the astrometric data extracted from the lightcurves using a sophisticated photometric model including the albedo map deduced from the space probe images. The

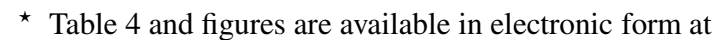
http://www. edpsciences.org. They are also available on the following ftp server together with the digitized lightcurves available as ASCII files at ftp://ftp.imcce.fr/pub/NSDC/jupiter/ raw_data/phenomena/mutual/1997/ aim of the present paper is to provide the photometric data and the observational parameters useful for future work on the improvement of dynamical models as well as of models of the surfaces of the satellites. These data will be available through the data base NSDC dedicated to the natural satellites (http://www. imcce.fr/nsdc).

\section{The mutual events}

The Earth and the Sun cross the equatorial plane of Jupiter every six years. The Jovian declinations of the Earth and the Sun become zero and since the orbital plane of the Galilean satellites is very close to the equatorial plane of Jupiter, the satellites occult and eclipse each other.

The 1997 period was favorable since it occurred during the opposition of Jupiter and the Sun.

Arlot (1996) made predictions of all the 1997 events using the G5 ephemerides based on Lieske's theory (Lieske 1977) of the motion of the Galilean satellites. 182 dates of mutual events were computed. Several campaigns of observations took place during the previous occurrences. Table 1 shows the results 
Table 1. Results of the past campaigns of observations.

\begin{tabular}{llll}
\hline \hline & 1985 & 1991 & 1997 \\
\hline Number of sites & 28 & 56 & 42 \\
Number of light curves & 166 & 374 & 275 \\
Number of observed events & 64 & 111 & 148 \\
\hline
\end{tabular}

obtained during each campaign. Our goal was to observe as many events as possible. Two observations of each event are needed to eliminate observational errors.

Since there is no thick atmosphere around the Galilean satellites, the photometric observations of such phenomena are very accurate for astrometric purposes. The results previously obtained after similar observations of the Galilean satellites (Arlot et al. 1997) show that an accuracy better than 30 mas can be expected.

This allows us to provide data necessary for the improvement of theoretical models of orbital motion and the determination of tide effects in the dynamics of Galilean satellites.

\section{The PHEMU97 campaign}

The observation of these phenomena required a coordinated international campaign in order to obtain a significant amount of data. These events occur over a short span of time so numerous observers located at several sites are necessary in order to avoid meteorological problems and observe from different longitudes to record different events. Thus observers previously involved in the PHEMU campaigns of observations of the mutual events of the Galilean satellites were invited to join the new campaign.

\subsection{Receivers}

For the observations of the mutual events only relative photometry is generally possible. Since the elevation of Jupiter above the horizon may be very small, the air mass is often too large and absolute photometry is not possible. The telescopes were equipped with the receivers listed in Table 2. Three kinds of receivers were used: photoelectric photometric single channel receivers, video cameras and two-dimensional CCD receivers. Visual observations are reported for comparison.

\subsection{Sites of observation}

This campaign, coordinated by the Institut de Mécanique Céleste, involved the different locations given in Table 3. This table gives the names, longitudes and latitudes of these sites.

\section{Lightcurve reduction procedure}

Lightcurves were derived from photometric measurements either with relative photometry performed with photoelectric photometers or with CCD cameras.

For observations made with CCD cameras in video mode the digitized signal was computed with digitizing boards. The lightcurves were obtained by aperture photometry, as were the observations for which a value of the diaphragm is provided. For CCD observations made in France, images were measured with the Gaussian photometry package of the ASTROL software (Colas 1996). Two dimensional measurements allow us to calibrate the signal of the satellite involved to the signal of a nearby satellite and to use data obtained under very difficult conditions (see for example Arlot et al. 1997).

The determination of the time of the minimum of light and of the value of the magnitude drop was based on a fit of the lightcurve with a sample polynomial. The errors in these determinations are also given. The error on the timing of the minimum is determined as follows: we calculate the noise in magnitude and transform it into a time error through the highest value of the decreasing speed in magnitude during the event. The largest errors occur for faint noisy events and the smallest for the fast ones. The errors are comparable only if the integration time is the same.

\section{The catalogue}

\subsection{The data}

Table 4 provides for each event (all dates are in UTC): - prediction of the time of the event:

1. Date of the event and its nature;

2. Start time of the event;

3. Maximum of the event;

4. End of the event;

5. Calculated magnitude drop;

6. Phase angle in degrees;

7. Apparent distance satellite-planet in planetary radii.

-for each observation of the event:

1. The site of observation;

2. -

3. The observed time of the maximum of magnitude drop and observational error;

4. -

5. The observed magnitude drop and observational error;

6. -

7. -

8. $(\mathrm{C}-\mathrm{O})$ of the observation in seconds of time; these quantities take into account the phase effect (Aksnes et al. 1986);

9. Aperture of the telescope in centimeters $(\mathrm{T}=$ reflector; $\mathrm{L}=$ refractor);

10. Code of the receiver used in column "Recept." (cf. Table 2);

11. Elevation of Jupiter above the horizon in degrees;

12. Elevation of the Sun above the horizon in degrees;

13. The observational conditions in column "Obs. cond.": [0] means no information, [1] means very good conditions, [2] means acceptable and [3] very difficult conditions;

14. The filter used, if any, in column "Filter"; no filter used is denoted by "-,";

15. The integration time of the measurements in seconds; a variable integration time is denoted "v";

16. Size of the diaphragm when used. 
Table 2. Receivers used for the observations.

\begin{tabular}{|c|c|c|}
\hline $\begin{array}{l}\text { Code as } \\
\text { given in } \\
\text { the tables }\end{array}$ & Description & reference \\
\hline \multirow[t]{2}{*}{ VISU } & Visual & $\begin{array}{l}\text { BARCELONA, } \\
\text { CATANIA2 (06-07-1997),CATANIA3 (03-08-1997) } \\
\text { LUMEZZANE (18-09-1997),MILANO,SEVILLA,MADRID }\end{array}$ \\
\hline & Single channel receivers & \\
\hline PM & EMI9893 & CATANIA1 (Blanco) \\
\hline PM1 & Photoelectric lp21 & LUMEZZANE \\
\hline PM2 & Photometer (RCA 4840) & PARIS \\
\hline PM3 & Hamamatsu R647-04 & FUNAHO,OKAYAMA \\
\hline PM4 & RCA931A & SAN-FERNANDO \\
\hline PM5 & UBV (Shugarov) & NAUCHNY (07-10) \\
\hline PM6 & Optec SSP3 Pin-Diode & ESSEN \\
\hline PMW & Photometer WBVR & NAUCHNY \\
\hline PMIR & Photometer IR & TENERIFE \\
\hline PM7 & Teloc II B channel photometric system & (CALERN) \\
\hline PM7 & Teloc II V channel photometric system & (CALERN) \\
\hline \multirow[t]{2}{*}{ PM7 } & Teloc II R channel photometric system & (CALERN) \\
\hline & Video receivers & \\
\hline $\mathrm{V}$ & Video OS25 (18-06) & PRAHA \\
\hline V1 & Video OS45D & PRAHA \\
\hline $\mathrm{V} 2$ & Ikegama B/W camera ICD-42E TypeF & WILP-ACHTERHOEK \\
\hline \multirow[t]{2}{*}{$\mathrm{V} 3$} & CCD MKII in video mode & MEUDON \\
\hline & CCD receivers & \\
\hline $\mathrm{CCD}$ & Starlight SX Xpress & CACERES,ZARAGOZA \\
\hline CCD1 & TH7863 & BORDEAUX,OHP \\
\hline $\mathrm{CCD} 2$ & TC-211 & BOWIE,ELLINBANK,NEW-YORK,SASSOEIROS,TORINO \\
\hline CCD3 & IRAC2 & ESO \\
\hline CCD4 & SPT-M102CE Sony B/W & LISBOA \\
\hline CCD5 & NXA $1001 / 03$ & REUX \\
\hline CCD6 & VC100-camera B/W & COMTHUREY \\
\hline CCD7 & $\mathrm{CCD} \mathrm{B} / \mathrm{W}$ camera & OOSTDUINKERKE \\
\hline CCD8 & KAF-6300 KODAK & UKKEL \\
\hline CCD10 & Hi-SIS11 & CHATEAUGIRON \\
\hline CCD11 & LYNX CCD camera $192 \times 168$ pixel-chip & STUTTGART \\
\hline CCD12 & 0.01 lux CCD video camera & TOPEKA \\
\hline CCDI & Hisis-22 & BUCHAREST1 (Vass),PIC-DU-MIDI \\
\hline CCDL & LYNX PC & ASHEVILLE \\
\hline CCDM & TC245 (IOC) & MUNICH \\
\hline CCDS & Chip CCD Sony & RAGUSA \\
\hline CCDW & Wright $1024 \times 1024$ & TENERIFE \\
\hline CCDST & ST-6V SBIG & ALMA-ATA,BUCHAREST2 (Stefanescu) \\
\hline CCDVX & HCS MXRII & BOSKOOP \\
\hline $\mathrm{CCDC}$ & $\begin{array}{l}\text { Thomson-CSF } 7862 \text { CDA chip } \\
\text { (photometrics CCD system) }\end{array}$ & KAVALUR \\
\hline
\end{tabular}

For each observation, a corresponding lightcurve is provided in Figs. 1 to 33 showing the magnitude drop versus UTC time scale.

These data and light-curves are available at the Natural Satellite Data Center (NSDC) server (http: //www . imcce. fr/nsdc).

\subsection{Discussion}

This catalogue provides observational information and reduced data issued from the PHEMU97 campaign. Another paper
(Vasundhara et al. 2003) provides the astrometric data extracted from the lightcurves.

The quality of each lightcurve may be judged either by the value of the errors on the determined parameters (time of the minimum of light and lightflux drop) or the appearance of the lightcurve itself.

As for the previous catalogues of such events, we computed the errors on the determined parameter as follows. The error on the lightflux drop is deduced from the standard deviation of the fit to the model light curve. The error on the date of the minimum is determined from the error on the magnitude drop combined with the speed of the decrease of the lightflux 
Table 3. Main sites of observation.

\begin{tabular}{|c|c|c|c|c|c|c|c|c|c|c|}
\hline \multirow[t]{2}{*}{ Main observatories } & \multirow{2}{*}{$\begin{array}{l}\text { Telescope } \\
\text { diameter } \\
\text { in } \mathrm{cm}\end{array}$} & \multicolumn{4}{|c|}{ Longitude } & \multicolumn{4}{|c|}{$\overline{\text { Latitude }}$} & \multirow{2}{*}{$\begin{array}{l}\text { elevation } \\
\text { meters }\end{array}$} \\
\hline & & $\circ$ & & " & & $\circ$ & ' & " & & \\
\hline ASHEVILLE (USA) & T20 & 82 & 25 & 45 & $\mathrm{~W}$ & 35 & 36 & 00 & $\mathrm{~N}$ & 670 \\
\hline ALMA-ATA (Kazakhstan) & T60 & 77 & 52 & 45 & $\mathrm{E}$ & 43 & 13 & 20 & $\mathrm{~N}$ & 2750 \\
\hline BARCELONA (Spain) & $\mathrm{T} 16$ & 02 & 12 & 07 & $\mathrm{E}$ & 41 & 25 & 18 & $\mathrm{~N}$ & 40 \\
\hline BORDEAUX (France) & T62 & 00 & 32 & 00 & W & 44 & 50 & 00 & $\mathrm{~N}$ & 73 \\
\hline BOSKOOP (The Netherlands) & $\mathrm{T} 30$ & 04 & 41 & 35 & $\mathrm{E}$ & 52 & 04 & 35 & $\mathrm{~N}$ & 0 \\
\hline BOWIE (USA) & $\mathrm{T} 20$ & 76 & 48 & 00 & $\mathrm{~W}$ & 39 & 00 & 00 & $\mathrm{~N}$ & 50 \\
\hline BUCAREST (Romania) & L38 & 26 & 05 & 46 & $\mathrm{E}$ & 44 & 24 & 50 & $\mathrm{~N}$ & 86 \\
\hline CACERES (Spain) & $\mathrm{T} 25$ & 06 & 23 & 00 & W & 39 & 27 & 00 & $\mathrm{~N}$ & 440 \\
\hline CALERN, OCA/CERGA (France) & $\mathrm{T} 150$ & 06 & 55 & 18 & $\mathrm{E}$ & 43 & 45 & 17 & $\mathrm{~N}$ & 1282 \\
\hline CATANIA1 (Italy) & T91 & 14 & 58 & 45 & $\mathrm{E}$ & 37 & 41 & 30 & $\mathrm{~N}$ & 1725 \\
\hline CATANIA2 (Italy) & $\mathrm{T} 20$ & 15 & 03 & 20 & $\mathrm{E}$ & 37 & 39 & 43 & $\mathrm{~N}$ & 300 \\
\hline CATANIA3 (Italy) & $\mathrm{T} 20$ & 14 & 57 & 40 & $\mathrm{E}$ & 37 & 39 & 43 & $\mathrm{~N}$ & 1250 \\
\hline CHATEAUGIRON (France) & $\mathrm{T} 21$ & 01 & 30 & 01 & $\mathrm{O}$ & 48 & 02 & 41 & $\mathrm{~N}$ & 70 \\
\hline COMTHUREY (Germany) & L18 & 13 & 11 & 31 & $\mathrm{E}$ & 53 & 16 & 04 & $\mathrm{~N}$ & 150 \\
\hline ELLINBANK (Australia) & T32 & 145 & 57 & 30 & $\mathrm{E}$ & 38 & 14 & 47 & $\mathrm{~S}$ & 138 \\
\hline ESO, La Silla (Chile) & $\mathrm{T} 220$ & 70 & 43 & 45 & W & 29 & 15 & 26 & S & 2200 \\
\hline ESSEN (Germany) & T36 & 07 & 04 & 15 & $\mathrm{E}$ & 41 & 24 & 35 & $\mathrm{~N}$ & 40 \\
\hline FUNAHO (Japan) & $\mathrm{T} 28$ & 33 & 42 & 43 & $\mathrm{E}$ & 34 & 34 & 43 & $\mathrm{~N}$ & 8 \\
\hline KAVALUR (India) & $\mathrm{T} 235$ & 78 & 49 & 49 & $\mathrm{E}$ & 12 & 34 & 49 & $\mathrm{~N}$ & 725 \\
\hline LISBOA (Portugal) & $\mathrm{T} 20$ & 09 & 07 & 42 & $\mathrm{~W}$ & 38 & 43 & 30 & $\mathrm{~N}$ & 96 \\
\hline LUMEZZANE (Italy) & $\mathrm{T} 40$ & 09 & 08 & 22 & $\mathrm{E}$ & 45 & 28 & 11 & $\mathrm{~N}$ & 122 \\
\hline MADRID (Spain) & - & 03 & 49 & 09 & W & 40 & 17 & 49 & $\mathrm{~N}$ & 680 \\
\hline MEUDON (France) & $\mathrm{T} 100$ & 02 & 14 & 00 & $\mathrm{E}$ & 48 & 48 & 00 & $\mathrm{~N}$ & 162 \\
\hline MILAN (Italy) & L08 & 09 & 07 & 22 & $\mathrm{E}$ & 45 & 27 & 19 & $\mathrm{~N}$ & 138 \\
\hline MUNCHEN (Germany & $\mathrm{T} 28$ & 11 & 34 & 30 & $\mathrm{E}$ & 48 & 11 & 17 & $\mathrm{~N}$ & 520 \\
\hline NAUCHNY (Crimea) & T60 & 34 & 01 & 00 & $\mathrm{E}$ & 44 & 43 & 37 & $\mathrm{~N}$ & 600 \\
\hline NEW-YORK (USA) & $\mathrm{T} 20$ & 78 & 00 & 00 & $\mathrm{~W}$ & 43 & 00 & 00 & $\mathrm{~N}$ & 200 \\
\hline OHP, Haute-Provence Obs. (France) & $\mathrm{T} 80$ & 05 & 43 & 00 & $\mathrm{E}$ & 43 & 56 & 00 & $\mathrm{~N}$ & 665 \\
\hline OKAYAMA (Japan) & T35 & 33 & 52 & 36 & $\mathrm{E}$ & 34 & 36 & 25 & $\mathrm{~N}$ & 3 \\
\hline OOSTDUINKERKE (Belgium) & $\mathrm{T} 25$ & 02 & 40 & 45 & $\mathrm{E}$ & 51 & 06 & 48 & $\mathrm{~N}$ & 4 \\
\hline PIC-DU-MIDI, OMP (France) & $\mathrm{T} 105$ & 00 & 09 & 00 & $\mathrm{E}$ & 42 & 56 & 00 & $\mathrm{~N}$ & 2861 \\
\hline PARIS (France) & L38 & 02 & 20 & 00 & $\mathrm{E}$ & 48 & 50 & 00 & $\mathrm{~N}$ & 67 \\
\hline PRAHA (Czech Republic) & L15 & 14 & 28 & 41 & $\mathrm{E}$ & 50 & 08 & 30 & $\mathrm{~N}$ & 325 \\
\hline RAGUSA (Italy) & $\mathrm{T} 20$ & 14 & 39 & 43 & $\mathrm{E}$ & 36 & 46 & 15 & $\mathrm{~N}$ & 25 \\
\hline REUX (Belgium) & $\mathrm{T} 30$ & 05 & 05 & 27 & $\mathrm{E}$ & 50 & 14 & 43 & $\mathrm{~N}$ & 317 \\
\hline SAN FERNANDO (Spain) & $\mathrm{T} 33$ & 06 & 12 & 21 & $\mathrm{~W}$ & 36 & 27 & 40 & $\mathrm{~N}$ & 30 \\
\hline SASSOEIROS (Portugal) & $\mathrm{T} 25$ & 09 & 19 & 34 & $\mathrm{~W}$ & 38 & 42 & 01 & $\mathrm{~N}$ & 64 \\
\hline SEVILLA (Spain) & $\mathrm{T} 21$ & 05 & 58 & 47 & $\mathrm{~W}$ & 37 & 23 & 40 & $\mathrm{~N}$ & 50 \\
\hline TENERIFE (Canarian Islands) & $\mathrm{T} 20$ & 16 & 30 & 38 & $\mathrm{~W}$ & 28 & 17 & 45 & $\mathrm{~N}$ & 2374 \\
\hline STUTTGART (Germany) & $\mathrm{T} 30$ & 09 & 08 & 15 & $\mathrm{E}$ & 48 & 42 & 00 & $\mathrm{~N}$ & 441 \\
\hline TOPEKA (USA) & L38 & 95 & 44 & 58 & $\mathrm{~W}$ & 39 & 00 & 03 & $\mathrm{~N}$ & 313 \\
\hline TORINO (Italy) & $\mathrm{T} 15$ & 07 & 38 & 10 & $\mathrm{E}$ & 45 & 02 & 51 & $\mathrm{~N}$ & 622 \\
\hline UCCLE (Belgium) & $\mathrm{T} 85$ & 04 & 21 & 29 & $\mathrm{E}$ & 50 & 47 & 55 & $\mathrm{~N}$ & 105 \\
\hline WILP-ACHTERHOEKE (The Netherlands) & $\mathrm{T} 20$ & 06 & 03 & 52 & $\mathrm{E}$ & 52 & 12 & 29 & $\mathrm{~N}$ & 6 \\
\hline ZARAGOZA (Spain) & $\mathrm{T} 15$ & 00 & 52 & 30 & $\mathrm{~W}$ & 41 & 38 & 46 & $\mathrm{~N}$ & 247 \\
\hline
\end{tabular}

during the event. This explains that this error depends on the number of points, on the integrating time and on the depth of the light curve. Because of that, the error bars may be compared only between events recorded with the same time constants and, preferably, with the same equipment in order to obtain the observational error and a measurement of the quality of the observation.

\section{Conclusion}

We give in this paper the results of the PHEMU97 campaign. To record the maximum of events, it was necessary to organize an international campaign. The phenomena recorded occur every 6 years and they lead to very accurate astrometric measurements which are very difficult to obtain with groundbased techniques. Such data may allow us to determine surface parameters by comparison of lightcurves with synthetic models.

Acknowledgements. These observations have been made possible by the CNRS (Centre National de la Recherche Scientifique), the INSU (Institut National des Sciences de l'Univers) and the CNES (Centre National d'Etudes Spatiales) through the PNP (Programme National de Planétologie) who supports the PHEMU97 campaign and the Institut de Mécanique Céleste et de Calcul des Éphémérides.

We also thank the staff of the observatories where these observations were made for their help during this campaign and Mrs Raoult for her help writing the paper. 


\section{References}

Aksnes, K., Franklin, F., \& Magnusson, P. 1986, AJ, 92, 1436

Arlot, J.-E., \& Thuillot, W. 1993, Icarus, 105, 427

Arlot, J.-E., \& Stavinschi, M. (eds.) 1996, PHESAT95 workshop, Suppl. Ann. Phys., 21, 1

Arlot, J.-E., \& Blanco, C. (eds.) 1999, PHEMU97 workshop, Institut de mécanique céleste, Paris

Arlot, J. E., Thuillot, W., Colas, F., Allet, C., \& Vu, D. T. 1989, Celest. Mech., 45, 129

Arlot, J.-E., et al. 1996, Proc. IAU Symp., 172, ed. S. Ferraz Mello, B. Morando, \& J.-E. Arlot (Kluwer Acad. Pub.), 423

Arlot, J.-E. and 70 observers 1997, A\&AS, 125, 399

Colas, F. 1996, in Proceedings of the PHESAT95 workshop, Supp. Ann. Phys., 21, 133

Dourneau, G. 1987, Thesis, Bordeaux University

Dourneau, G. 1993, A\&A, 267, 292

Duriez, L., \& Vienne, A. 1991, A\&A, 243, 263

Emelianov, N. V., et al. 1997, Sol. System Res., 31, 436

Emelianov, N. V., et al. 1999, A\&AS, 139, 47

Froeschlé, et al. 1988, A\&AS, 262, 308

Le Campion, J.-F., Montignac, G., Chauvet, F., et al. 1992, A\&A, 266, 568

Soma, M. 1992, A\&A, 265, L21

Strugnell, P. R., \& Taylor, D. B. 1990, 83, 289

Thuillot, W., Arlot, J.-E., Colas, F., et al. 1996, Proceedings of the Colloquium Journées Systèmes de Référence, DANOF, Paris Observatory, 34

Vasundhara, R., Arlot, J. E., Lainey, V., \& Thuillot, W. 2003, A\&A, 410,337

Vienne, A., \& Duriez, L. 1991, A\&A, 246, 619

Vienne, A., \& Duriez, L. 1992, A\&A, 257, 331

Vienne, A., \& Duriez, L. 1995, A\&A, 297, 588

1 Institut de mécanique céleste et de calcul des éphémérides, Observatoire de Paris, UMR 8028 CNRS, 77 avenue DenfertRochereau, 75014 Paris, France

e-mail: Jean-Eudes.Arlot@imcce.fr

2 Funaho, Japan

3 Dipartimento di Fisica e Astronomia dell'Universita di Catania, Italy

${ }^{4}$ European Southern Observatory, La Silla, Chile
5 Observatoire de Bordeaux, France

${ }^{6}$ Observatoire de la Côte d'Azur, France

7 Observatoire de Paris, France

8 Institutul Astronomic, Bucuresti, Romania

9 Krimean Laboratory of the Sternberg Astronomical Institute, Moscow, Russia

${ }_{10}$ Fesenkov Astrophysical Institute, Almaty, Kazakhstan

11 Sternberg Astronomical Institute, Moscow, Russia

12 Milano, Italy

13 GSF, Munchen, Germany

14 Observatorio S. Zani, Lumezzane, Italy

15 Stuttgart, Germany

16 Buinen, Netherlands

17 Reux, Belgium

18 Boskoop, The Netherlands

19 IAC, Tenerife, Spain

20 WWC, Asheville, USA

${ }^{21}$ ORB, Bruxelles, Belgium

22 Essen, Germany

${ }^{23}$ San Fernando, Spain

${ }^{24}$ Sevilla, Spain

25 Chateaugiron, France

26 Marwitz, Germany

27 Universidade de Extramadura, Badajoz, Spain

28 Lisboa, Portugal

29 Planetarium Praha, Czech Rep.

30 GAS, Zaragossa, Spain

31 Okayama, Japan

${ }^{32}$ Oosterduinkerke, Belgium

33 OCA, Nice, France

34 IIA, Bangalore, India

35 Madrid, Spain

${ }^{36}$ OAIN, Scicli, Ragusa, Italy

37 Topeka, Kansas, USA

38 Ellinbank, Australia

39 Farmington, New-York, USA

40 GAC, Catania, Italy

41 Barcelona, Spain

42 Bowie, Maryland, USA

43 Belgium

44 Tokyo, Japan 


\section{Online Material}


J.-E. Arlot et al.: The PHEMU97 Campaign, Online Material p 2

Table 4. Observed data.

\begin{tabular}{|c|c|c|c|c|c|c|c|c|c|c|c|c|c|c|c|}
\hline $\begin{array}{c}\text { Dates } \\
\text { Phenomena } \\
\text { Locations } \\
\text { (1) } \\
\end{array}$ & $\begin{array}{c}\text { Begins } \\
\mathrm{h} \mathrm{m} \mathrm{s} \\
\text { (2) } \\
\end{array}$ & $\begin{array}{l}\text { Maxi. } \\
\text { h m s } \\
\text { (3) } \\
\end{array}$ & $\begin{array}{c}\text { Ends } \\
\text { h m s } \\
\text { (4) } \\
\end{array}$ & $\begin{array}{c}\text { Magn. } \\
\text { drop } \\
\text { (5) }\end{array}$ & $\begin{array}{l}\text { Ph. } \\
\text { (s) } \\
(6) \\
\end{array}$ & $\begin{array}{l}\text { Dist. } \\
(\mathbf{R j}) \\
(7) \\
\end{array}$ & $\begin{array}{c}\text { C-O } \\
(\mathrm{s}) \\
(8) \\
\end{array}$ & $\begin{array}{c}\text { Ap. } \\
\text { (cm) } \\
(9) \\
\end{array}$ & $\begin{array}{l}\text { Rec. } \\
\text { (10) }\end{array}$ & $\begin{array}{c}\text { El. } \\
\text { Jup. } \\
\left({ }^{\circ}\right. \\
(11) \\
\end{array}$ & $\begin{array}{l}\text { El. } \\
\text { Sun } \\
\left({ }^{\circ}\right) \\
(12) \\
\end{array}$ & $\begin{array}{l}\text { Cd. } \\
\text { (13) }\end{array}$ & $\begin{array}{l}\text { Filt. } \\
\text { (14) }\end{array}$ & $\begin{array}{l}\text { T. } \\
\text { int. } \\
\text { (s) } \\
\text { (15) } \\
\end{array}$ & $\begin{array}{l}\text { Dia. } \\
\text { (") } \\
(16) \\
\end{array}$ \\
\hline $\begin{array}{r}96 / 6 / 8 \\
102(P) \\
\end{array}$ & 13851 & $148 \quad 32$ & 15833 & . 040 & -14.2 & 2.0 & & & & & & & & & \\
\hline OHP & & $\begin{array}{rr}150 & 6 \\
& \pm 199 \\
\end{array}$ & & $\begin{array}{r}.111 \\
\pm .067 \\
\end{array}$ & & & -94 & T 80. & CCD1 & 24 & -17 & 3 & - & - & - \\
\hline $\begin{array}{l}97 / 4 / 24 \\
402(\mathrm{~T}) \\
\end{array}$ & 35231 & $355 \quad 48$ & 3596 & .295 & 5.7 & 4.4 & & & & & & & & & \\
\hline & & $\begin{array}{ll}355 & 59 \\
& \pm 6\end{array}$ & & $\begin{array}{r}1.346 \\
\pm .067\end{array}$ & & & -11 & T 62. & CCD1 & 14 & -11 & 2 & $R$ & 2. & - \\
\hline OHP & & $\begin{array}{lll}3 & 55 & 56 \\
& \pm 4\end{array} \mid$ & & $\begin{array}{r}1.289 \\
+.064\end{array}$ & & & -8 & Т 80. & CCD1 & 18 & -8 & 2 & I & 1. & - \\
\hline ZARAGOZA & & $\begin{array}{ll}355 & 57 \\
& \pm 14\end{array}$ & & $\begin{array}{l}.817 \\
\pm .159\end{array}$ & & & -9 & Т 15. & CCD & 11 & -18 & 1 & - & 10. & - \\
\hline $\begin{array}{l}\text { MADRID } \\
\text { MUNICH }\end{array}$ & & $\begin{array}{ccc}356 & 21 \\
& \pm 3 \\
356 & 1 \\
& \pm 15 \\
\end{array}$ & & $\begin{array}{l}5.548 \\
\pm .245 \\
1.225 \\
\pm .208 \\
\end{array}$ & & & $\begin{array}{l}-33 \\
-13\end{array}$ & $\begin{array}{l}\text { Т } 0 . \\
\text { Т } 28 .\end{array}$ & $\begin{array}{l}\text { VISU } \\
\text { CCDM }\end{array}$ & $\begin{array}{l}15 \\
17\end{array}$ & $\begin{array}{l}-16 \\
-2\end{array}$ & $\begin{array}{l}1 \\
1\end{array}$ & $\begin{array}{l}- \\
* 4\end{array}$ & - & - \\
\hline $\begin{array}{l}97 / 4 / 25 \\
403(P) \\
\end{array}$ & 24349 & $\begin{array}{l}315 \quad 39 \\
\end{array}$ & 34819 & .187 & -87.5 & 5.3 & & & & & & & & & \\
\hline $\begin{array}{c}\text { OHP } \\
\text { CATANIA }\end{array}$ & & $\begin{array}{ccc}316 & 17 \\
& \pm 443 \\
319 & 45 \\
& \pm 102 \\
\end{array}$ & & $\begin{array}{r}.367 \\
\pm .129 \\
.038 \\
\pm .005 \\
\end{array}$ & & & $\begin{array}{l}-38 \\
122\end{array}$ & $\begin{array}{l}\text { T } 80 . \\
\text { Т } 91 .\end{array}$ & $\begin{array}{c}\text { CCD1 } \\
\text { PM }\end{array}$ & $\begin{array}{l}13 \\
22\end{array}$ & $\begin{array}{l}-14 \\
-11\end{array}$ & $\begin{array}{l}3 \\
2\end{array}$ & $\begin{array}{l}\text { I } \\
V\end{array}$ & $\begin{array}{l}2 . \\
0.1\end{array}$ & - \\
\hline $\begin{array}{r}97 / 5 / 2 \\
2 \mathrm{E1}(\mathrm{p}) \\
\end{array}$ & 223646 & $2238 \quad 28$ & 224012 & . 028 & -3.3 & 4.9 & & & & & & & & & \\
\hline KAVALUR & & $\begin{array}{rc}2238 & 1 \\
& \pm 35 \\
\end{array}$ & & $\begin{array}{r}.044 \\
\pm .022 \\
\end{array}$ & & & 28 & T 102. & CCDC & 39 & -25 & 3 & $* 3$ & - & \\
\hline $\begin{array}{l}97 / 5 / 12 \\
403(P) \\
\end{array}$ & 23338 & $237 \quad 39$ & 24140 & . 166 & 10.8 & 6.9 & & & & & & & & & \\
\hline $\begin{array}{l}\text { BOSKOOP } \\
\text { MUNICH }\end{array}$ & & $\begin{array}{cc}237 & 36 \\
& \pm 119 \\
2 & 37 \\
& 34 \\
\pm 36 \\
\end{array}$ & & $\begin{array}{c}.119 \\
\pm .102 \\
.301 \\
\pm .104 \\
\end{array}$ & & & 3 & $\begin{array}{l}\text { Т } 30 . \\
\text { Т } 28 .\end{array}$ & $\begin{array}{l}\text { CCDVX } \\
\text { CCDM }\end{array}$ & $\begin{array}{l}11 \\
20\end{array}$ & $\begin{array}{l}-10 \\
-7\end{array}$ & $\begin{array}{l}2 \\
2\end{array}$ & $*_{4}$ & 0.04 & - \\
\hline $\begin{array}{r}97 / 5 / 13 \\
203(\mathrm{~A}) \\
\end{array}$ & 22015 & $223 \quad 29$ & 22644 & .479 & 9.6 & 5.6 & & & & & & & & & \\
\hline RAGUSA & & $\begin{array}{cc}233 & 4 \\
& \pm 38 \\
\end{array}$ & & $\begin{array}{r}.280 \\
\pm .076 \\
\end{array}$ & & & -575 & Т 25. & CCDS & 25 & -16 & 2 & v & 0.2 & - \\
\hline $\begin{array}{l}97 / 5 / 15 \\
301(P) \\
\end{array}$ & 20576 & $210 \quad 8$ & $\begin{array}{llll}2 & 3 & 10\end{array}$ & .152 & 7.3 & 4.8 & & & & & & & & & \\
\hline KAVALUR & & $\begin{array}{rr}2059 \quad 45 \\
& \pm 3 \\
\end{array}$ & & $\begin{array}{r}.207 \\
\pm .006 \\
\end{array}$ & & & 23 & T 235. & $\mathrm{CCDC}$ & 28 & -43 & 1 & $* 3$ & 2. & - \\
\hline $\begin{array}{r}97 / 5 / 21 \\
304(P) \\
\end{array}$ & 82626 & $844 \quad 27$ & $\begin{array}{lll}9 & 2 & 5 \\
\end{array}$ & .279 & 39.6 & 14.6 & & & & & & & & & \\
\hline ASHEVILLE & & $\begin{array}{rr}843 & 28 \\
& \pm 62 \\
\end{array}$ & & $\begin{array}{r}.168 \\
\pm .026 \\
\end{array}$ & & & 59 & Т 20. & CCDL & 29 & -17 & 2 & v & 0.5 & 20. \\
\hline $\begin{array}{l}97 / 5 / 29 \\
1 \mathrm{E} 2(\mathrm{P}) \\
\end{array}$ & $\begin{array}{lll}1 & 6 & 58 \\
\end{array}$ & $19 \quad 50$ & 11242 & .809 & -4.8 & 5.8 & & & & & & & & & \\
\hline $\begin{array}{c}\text { LUMEZZANE } \\
\text { BOOSKOOP } \\
\text { MUNICH }\end{array}$ & & $\begin{array}{ccc}1 & 11 & 0 \\
& & \pm 10 \\
1 & 10 & 2 \\
& & \pm 9 \\
1 & 10 & 4 \\
& & \pm 4 \\
\end{array}$ & & $\begin{array}{l}.477 \\
\pm .087 \\
1.641 \\
\pm .377 \\
1.945 \\
\pm .156 \\
\end{array}$ & & & $\begin{array}{l}-70 \\
-12 \\
-14\end{array}$ & $\begin{array}{l}\text { T } 40 . \\
\text { T } 30 . \\
\text { T } 28 .\end{array}$ & $\begin{array}{c}\text { PM1 } \\
\text { CCDVX } \\
\text { CCDM }\end{array}$ & $\begin{array}{l}15 \\
8 \\
13\end{array}$ & $\begin{array}{l}-18 \\
-14 \\
-15\end{array}$ & $\begin{array}{l}3 \\
2 \\
1\end{array}$ & $\begin{array}{l}V \\
- \\
* 4\end{array}$ & $\begin{array}{l}0.5 \\
0.04\end{array}$ & $\begin{array}{l}- \\
- \\
-\end{array}$ \\
\hline $\begin{array}{l}97 / 5 / 29 \\
302(P) \\
\end{array}$ & $233 \quad 16$ & $236 \quad 60$ & 231044 & .228 & 6.7 & 7.6 & & & & & & & & & \\
\hline KAVALUR & & 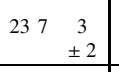 & & $\begin{array}{r}.566 \\
\pm .009 \\
\end{array}$ & & & -3 & Т 235. & CCDC & 60 & -16 & 1 & *3 & 2. & - \\
\hline $\begin{array}{l}97 / 5 / 30 \\
301(\mathrm{~T}) \\
\end{array}$ & $\begin{array}{lll}3 & 0 & 18 \\
\end{array}$ & $\begin{array}{lll}3 & 5 & 18 \\
\end{array}$ & 31022 & .321 & 10.1 & 5 & & & & & & & & & \\
\hline $\begin{array}{c}\text { PARIS } \\
\text { RAGUSA } \\
\text { TORINO } \\
\text { BORDEAUX } \\
\text { OHP }\end{array}$ & & 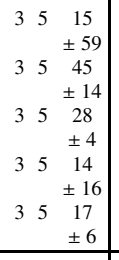 & & $\begin{array}{c}.358 \\
\pm .122 \\
.962 \\
. .157 \\
.551 \\
\pm .210 \\
.728 \\
\pm .064 \\
.665 \\
\pm .033 \\
\end{array}$ & & & $\begin{array}{c}3 \\
-27 \\
-10 \\
4 \\
1\end{array}$ & $\begin{array}{l}\text { L } 38 . \\
\text { T } 25 . \\
\text { T } 15 .\end{array}$ & $\begin{array}{l}\text { PM2 } \\
\text { CCDS } \\
\text { CCD2 } \\
\text { CCD1 } \\
\text { CCD1 }\end{array}$ & $\begin{array}{l}22 \\
36 \\
27 \\
14 \\
27\end{array}$ & $\begin{array}{l}-7 \\
-7 \\
-6 \\
-11 \\
-8\end{array}$ & $\begin{array}{l}2 \\
1 \\
2\end{array}$ & $\begin{array}{l}V \\
R \\
- \\
R\end{array}$ & $\begin{array}{l}0.3 \\
0.2 \\
3-6 \\
3-2\end{array}$ & $\begin{array}{l}- \\
- \\
-\end{array}$ \\
\hline
\end{tabular}


J.-E. Arlot et al.: The PHEMU97 Campaign, Online Material p 3

Table 4. continued.

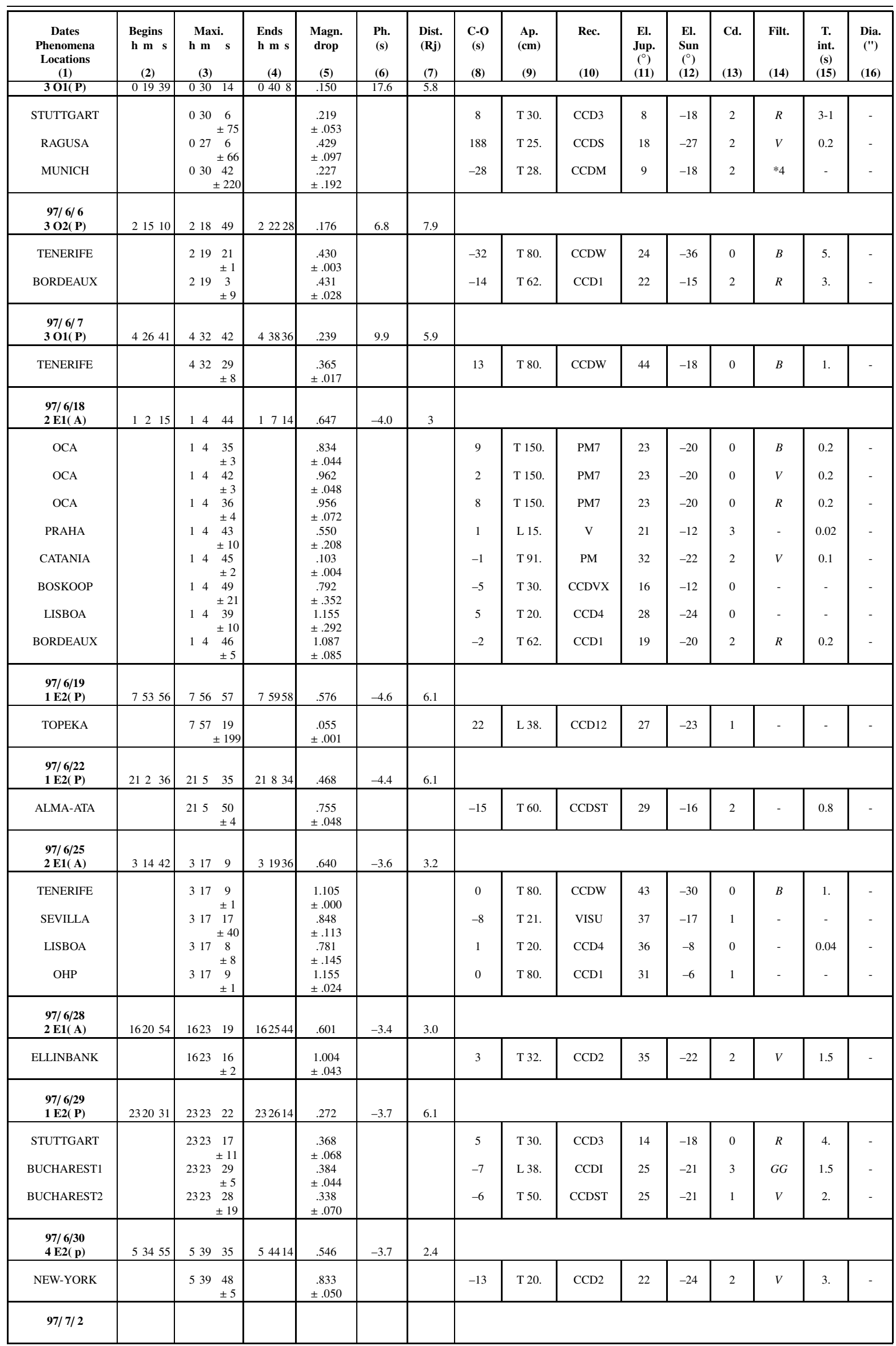


J.-E. Arlot et al.: The PHEMU97 Campaign, Online Material p 4

Table 4. continued.

\begin{tabular}{|c|c|c|c|c|c|c|c|c|c|c|c|c|c|c|c|}
\hline $\begin{array}{c}\text { Dates } \\
\text { Phenomena } \\
\text { Locations } \\
\text { (1) }\end{array}$ & $\begin{array}{c}\text { Begins } \\
\mathrm{h} \mathrm{m} \mathrm{s} \\
\text { (2) }\end{array}$ & $\begin{array}{c}\underset{\text { h m }}{\text { Maxi. }} \\
\text { (3) }\end{array}$ & $\begin{array}{c}\begin{array}{c}\text { Ends } \\
\text { h m s }\end{array} \\
\text { (4) }\end{array}$ & $\begin{array}{c}\text { Magn. } \\
\text { drop } \\
(5)\end{array}$ & $\begin{array}{l}\text { Ph. } \\
\text { (s) } \\
\text { (6) }\end{array}$ & $\begin{array}{l}\text { Dist. } \\
(\mathbf{R j})\end{array}$ & $\begin{array}{c}\text { C-O } \\
(\mathrm{s}) \\
(8)\end{array}$ & $\begin{array}{l}\text { Ap. } \\
\text { (cm) } \\
(9)\end{array}$ & $\begin{array}{l}\text { Rec. } \\
\text { (10) }\end{array}$ & $\begin{array}{l}\text { El. } \\
\text { Jup. } \\
\left({ }^{\circ}\right) \\
(11)\end{array}$ & $\begin{array}{l}\text { El. } \\
\text { Sun } \\
\left({ }^{\circ}\right) \\
(12)\end{array}$ & $\begin{array}{l}\text { Cd. } \\
\text { (13) }\end{array}$ & $\begin{array}{l}\text { Filt. } \\
\text { (14) }\end{array}$ & $\begin{array}{l}\text { T. } \\
\text { int. } \\
\text { (s) } \\
(15)\end{array}$ & $\begin{array}{l}\text { Dia. } \\
\text { (") } \\
\text { (16) }\end{array}$ \\
\hline $2 \mathrm{E1}(\mathrm{A})$ & \begin{tabular}{|l|l|}
5273 \\
\end{tabular} & \begin{tabular}{ll|}
529 & 25 \\
\end{tabular} & 53147 & .539 & -3.1 & 2.8 & & & & & & & & & \\
\hline TOPEKA & & $\begin{array}{rr}530 & 48 \\
& \pm 35 \\
\end{array}$ & & $\begin{array}{r}.030 \\
\pm .008 \\
\end{array}$ & & & 83 & L 38. & CCD12 & 14 & -27 & 1 & - & & - \\
\hline $\begin{array}{r}97 / 7 / 4 \\
302(P) \\
\end{array}$ & 143924 & $1443 \quad 24$ & 144725 & .123 & 5.5 & 8.7 & & & & & & & & & \\
\hline OKAYAMA & & $\begin{array}{cc}1443 & 28 \\
\pm 29\end{array}$ & & $\begin{array}{r}.249 \\
\pm .061\end{array}$ & & & -4 & Т 35. & PM3 & 23 & -32 & 1 & V & 0.2 & 40. \\
\hline $\begin{array}{r}97 / 7 / 5 \\
301(T) \\
\end{array}$ & 155742 & 1614 & 16424 & .321 & 3.6 & 4.4 & & & & & & & & & \\
\hline ELLINBANK & & $\begin{array}{cc}160 & 58 \\
& \pm 4 \\
\end{array}$ & & $\begin{array}{r}.532 \\
\pm .026 \\
\end{array}$ & & & 6 & Т 32. & $\mathrm{CCD} 2$ & 35 & -25 & 2 & V & 1. & - \\
\hline $\begin{array}{r}97 / 7 / 6 \\
3 \mathrm{E} 4(\mathbf{P}) \\
\end{array}$ & 221818 & $2232 \quad 40$ & 224711 & .547 & -15.3 & 17.3 & & & & & & & & & \\
\hline $\begin{array}{c}\text { CATANIA2 } \\
\text { SEVILLA } \\
\text { TORINO }\end{array}$ & & $\begin{array}{cc}2233 & 36 \\
& \pm 18 \\
2234 & 24 \\
& \pm 50 \\
2232 & 50 \\
\pm 36 \\
\end{array}$ & & $\begin{array}{c}.318 \\
\pm .023 \\
1.269 \\
\pm .530 \\
.796 \\
\pm .077 \\
\end{array}$ & & & $\begin{array}{l}-56 \\
-104 \\
-10\end{array}$ & $\begin{array}{l}\text { Т } 20 . \\
\text { T } 21 . \\
\text { T } 15 .\end{array}$ & $\begin{array}{l}\text { VISU } \\
\text { VISU } \\
\text { CCD2 }\end{array}$ & $\begin{array}{l}22 \\
9 \\
14\end{array}$ & $\begin{array}{l}-29 \\
-24 \\
-21\end{array}$ & $\begin{array}{l}2 \\
1 \\
2\end{array}$ & - & 5. & - \\
\hline $\begin{array}{l}97 / 7 / 7 \\
1 \mathrm{E} 2(\mathrm{p}) \\
\end{array}$ & 13936 & $142 \quad 14$ & 14452 & .116 & -2.7 & & & & & & & & & & \\
\hline $\begin{array}{c}\text { TENERIFE } \\
\text { SAN-FERN. } \\
\text { LISBOA } \\
\text { BORDEAUX }\end{array}$ & & $\begin{array}{ccc}142 & 33 \\
& & \pm 0 \\
142 & 33 \\
& & \pm 62 \\
1 & 42 & 21 \\
& & \pm 20 \\
1 & 42 & 31 \\
& & \pm 19 \\
& & \\
\end{array}$ & & $\begin{array}{l}.156 \\
\pm .000 \\
.227 \\
\pm .377 \\
.153 \\
.046 \\
.126 \\
\pm .030 \\
\end{array}$ & & & $\begin{array}{l}-19 \\
-19 \\
-7 \\
-17\end{array}$ & $\begin{array}{l}\text { T } 80 . \\
\text { T } 33 . \\
\text { T } 25 . \\
\text { T } 62 .\end{array}$ & $\begin{array}{l}\mathrm{CCDW} \\
\mathrm{PM} 4 \\
\mathrm{CCD} 2 \\
\mathrm{CCD} 1\end{array}$ & $\begin{array}{l}38 \\
35 \\
32 \\
29\end{array}$ & $\begin{array}{l}-39 \\
-28 \\
-27 \\
-19\end{array}$ & $\begin{array}{l}0 \\
0 \\
0 \\
2\end{array}$ & $\begin{array}{l}B \\
- \\
- \\
\end{array}$ & 1. & - \\
\hline $\begin{array}{r}97 / 7 / 9 \\
2 \mathrm{E} 3(\mathrm{p}) \\
\end{array}$ & 0133 & $016 \quad 22$ & $\begin{array}{llll}0 & 1941 \\
\end{array}$ & .095 & -3.7 & 1.5 & & & & & & & & & \\
\hline $\begin{array}{l}\text { SEVILLA } \\
\text { REUX }\end{array}$ & & $\begin{array}{ccc}0 & 17 & 41 \\
& \pm 21 \\
0 & 16 & 27 \\
& \pm 193\end{array}$ & & $\begin{array}{c}.017 \\
\pm .002 \\
.043 \\
\pm .269 \\
\end{array}$ & & & $\begin{array}{l}-79 \\
-5\end{array}$ & $\begin{array}{l}\text { Т } 21 . \\
\text { Т } 30 .\end{array}$ & $\begin{array}{l}\text { VISU } \\
\text { CCD5 }\end{array}$ & $\begin{array}{l}26 \\
20\end{array}$ & $\begin{array}{l}-30 \\
-17\end{array}$ & $\begin{array}{l}1 \\
0\end{array}$ & - & 0.04 & - \\
\hline $\begin{array}{l}97 / 7 / 12 \\
2 \text { E1 }(P)\end{array}$ & 204535 & $2047 \quad 45$ & 204953 & .297 & -2.2 & 2.3 & & & & & & & & & \\
\hline KAVALUR & & $\begin{array}{rr}2047 & 43 \\
& \pm 3 \\
\end{array}$ & & $\begin{array}{r}.254 \\
\pm .012 \\
\end{array}$ & & & 1 & Т 235. & $\mathrm{CCDC}$ & 62 & -45 & 1 & *3 & - & - \\
\hline $\begin{array}{r}97 / 7 / 15 \\
103(P) \\
\end{array}$ & 185542 & $1858 \quad 12$ & 19042 & .169 & 3.5 & 3.1 & & & & & & & & & \\
\hline ALMA-ATA & & $\begin{array}{cc}1856 & 6 \\
& \pm 14 \\
\end{array}$ & & $\begin{array}{r}.132 \\
\pm .025 \\
\end{array}$ & & & 126 & $\mathrm{~T} 60$. & CCDST & 26 & -25 & 2 & - & 0.8 & - \\
\hline $\begin{array}{r}97 / 7 / 16 \\
2 \text { E3(A) } \\
\end{array}$ & 32516 & $328 \quad 57$ & 33237 & .208 & -3.4 & 1.5 & & & & & & & & & \\
\hline $\begin{array}{l}\text { TENERIFE } \\
\text { SAN-FERN. }\end{array}$ & & $\begin{array}{cc}328 & 58 \\
& \pm 5 \\
330 & 8 \\
& \pm 4 \\
\end{array}$ & & $\begin{array}{r}.214 \\
\pm .013 \\
2.153 \\
\pm .443 \\
\end{array}$ & & & -71 & т 33. & $\begin{array}{l}\text { CCDW } \\
\text { PM4 }\end{array}$ & 35 & $\begin{array}{l}-30 \\
-18\end{array}$ & 0 & $B$ & 1. & - \\
\hline $\begin{array}{r}97 / 7 / 18 \\
3 \mathrm{E} 2(\mathrm{p}) \\
\end{array}$ & 185131 & $1856 \quad 37$ & $\begin{array}{llll}19 & 1 & 43 \\
\end{array}$ & .240 & -3.1 & 8.6 & & & & & & & & & \\
\hline $\begin{array}{l}\text { ALMA-ATA } \\
\text { KAVALUR }\end{array}$ & & $\begin{array}{cc}1856 & 44 \\
& \pm 21 \\
& \pm 21 \\
1856 & 55 \\
& \pm 5 \\
\end{array}$ & & $\begin{array}{c}.222 \\
\pm .033 \\
.232 \\
\pm .010 \\
\end{array}$ & & & $\begin{array}{l}-6 \\
-17\end{array}$ & $\begin{array}{l}\text { T } 60 . \\
\text { T } 102 .\end{array}$ & $\begin{array}{l}\text { CCDST } \\
\text { CCDC }\end{array}$ & $\begin{array}{l}27 \\
54\end{array}$ & $\begin{array}{l}-26 \\
-56\end{array}$ & $\begin{array}{l}0 \\
0\end{array}$ & $\begin{array}{c}2 \\
1 * 3\end{array}$ & 0.8 & \\
\hline $\begin{array}{r}97 / 7 / 18 \\
302(P) \\
\end{array}$ & 204249 & $2047 \quad 48$ & 205248 & .182 & 4.1 & 9.1 & & & & & & & & & \\
\hline ALMA-ATA & & $\begin{array}{cc}2048 & 46 \\
& \pm 4 \\
2047 & 55 \\
& \pm 7 \\
\end{array}$ & & $\begin{array}{r}1.325 \\
\pm .028 \\
.390 \\
\pm .018 \\
\end{array}$ & & & $\begin{array}{l}-58 \\
-7\end{array}$ & $\begin{array}{l}\text { T } 15 . \\
\text { T } 60 .\end{array}$ & $\begin{array}{l}\mathrm{CCD} 2 \\
\mathrm{CCDST}\end{array}$ & $\begin{array}{r}6 \\
31\end{array}$ & $\begin{array}{l}-14 \\
-21\end{array}$ & $\begin{array}{l}0 \\
0\end{array}$ & $\begin{array}{l}2 \\
2\end{array}$ & $\begin{array}{l}3.5 \\
0.5\end{array}$ & \\
\hline $\begin{array}{r}97 / 7 / 19 \\
301(P)\end{array}$ & 205628 & $2059 \quad 23$ & $\begin{array}{llll}2 & 2 & 17\end{array}$ & .293 & 2.1 & 3.4 & & & & & & & & & \\
\hline $\begin{array}{c}\text { TORINO } \\
\text { ALMA-ATA } \\
\text { OHP }\end{array}$ & & $\begin{array}{cc}2059 & 17 \\
& \pm 10 \\
2059 & 15 \\
& \pm 5 \\
2059 & 15 \\
& \pm 11\end{array}$ & & $\begin{array}{c}.977 \\
\pm .102 \\
.520 \\
\pm .036 \\
.620 \\
\pm .078\end{array}$ & & & $\begin{array}{l}8 \\
8\end{array}$ & $\begin{array}{l}\text { Т } 15 . \\
\text { Т } 60 . \\
\text { Т } 80 .\end{array}$ & $\begin{array}{l}\text { CCD2 } \\
\text { CCDST } \\
\text { CCD1 }\end{array}$ & $\begin{array}{l}8 \\
30 \\
23\end{array}$ & $\begin{array}{l}-16 \\
-20 \\
-25\end{array}$ & $\begin{array}{l}0 \\
0\end{array}$ & $\begin{array}{l}2 \\
2 \\
2\end{array}$ & $\begin{array}{l}2 . \\
0.5\end{array}$ & - \\
\hline
\end{tabular}


J.-E. Arlot et al.: The PHEMU97 Campaign, Online Material p 5

Table 4. continued.

\begin{tabular}{|c|c|c|c|c|c|c|c|c|c|c|c|c|c|c|c|}
\hline $\begin{array}{c}\text { Dates } \\
\text { Phenomena } \\
\text { Locations } \\
\text { (1) }\end{array}$ & $\begin{array}{c}\text { Begins } \\
\mathrm{h} \mathrm{m} \mathrm{s} \\
\text { (2) }\end{array}$ & $\begin{array}{l}\text { Maxi. } \\
\text { h m s } \\
\text { (3) }\end{array}$ & $\begin{array}{c}\text { Ends } \\
\text { h m s } \\
\text { (4) }\end{array}$ & $\begin{array}{c}\text { Magn. } \\
\text { drop }\end{array}$ & $\begin{array}{l}\text { Ph. } \\
\text { (s) } \\
\text { (6) }\end{array}$ & $\begin{array}{l}\text { Dist. } \\
(\mathbf{R j})\end{array}$ & $\begin{array}{l}\text { C-O } \\
\text { (s) }\end{array}$ & $\begin{array}{c}\text { Ap. } \\
\text { (cm) } \\
(9)\end{array}$ & $\begin{array}{l}\text { Rec. } \\
\text { (10) }\end{array}$ & $\begin{array}{c}\text { El. } \\
\text { Jup. } \\
\left({ }^{\circ}\right) \\
(11)\end{array}$ & $\begin{array}{l}\text { El. } \\
\text { Sun } \\
\left({ }^{\circ}\right) \\
(12)\end{array}$ & $\begin{array}{l}\text { Cd. } \\
\text { (13) }\end{array}$ & $\begin{array}{l}\text { Filt. } \\
\text { (14) }\end{array}$ & $\begin{array}{l}\text { T. } \\
\text { int. } \\
\text { (s) } \\
\text { (15) }\end{array}$ & $\begin{array}{l}\text { Dia. } \\
\text { (") } \\
\text { (16) }\end{array}$ \\
\hline $\begin{array}{l}97 / 7 / 19 \\
2 \mathrm{E1}(\mathrm{p}) \\
\end{array}$ & 225757 & $2259 \quad 54$ & 23150 & .149 & -1.4 & 1.9 & & & & & & & & & \\
\hline $\begin{array}{c}\text { REUX } \\
\text { BORDEAUX }\end{array}$ & & $\begin{array}{cc}230 & 8 \\
& \pm 112 \\
2259 & 22 \\
& \pm 49 \\
\end{array}$ & & $\begin{array}{c}.115 \\
\pm .313 \\
.087 \\
\pm .069 \\
\end{array}$ & & & -14 & $\begin{array}{l}\text { Т } 30 . \\
\text { Т } 62 .\end{array}$ & $\begin{array}{l}\text { CCD5 } \\
\text { CCD1 }\end{array}$ & $\begin{array}{l}17 \\
19\end{array}$ & $\begin{array}{l}-18 \\
-23\end{array}$ & $\begin{array}{l}0 \\
0\end{array}$ & $R$ & $\begin{array}{c}0.04 \\
0.5\end{array}$ & - \\
\hline $\begin{array}{r}97 / 7 / 23 \\
2 \mathbf{E 3}(\mathbf{A}) \\
\end{array}$ & 63743 & $641 \quad 35$ & 64527 & .313 & -2.7 & 1.4 & & & & & & & & & \\
\hline TOPEKA & & $\begin{array}{rr}640 & 56 \\
& \pm 151\end{array}$ & & $\begin{array}{c}.042 \\
\pm .017\end{array}$ & & & 0 & L 38. & CCD12 & 33 & -31 & 0 & 1 & - & - \\
\hline $\begin{array}{l}97 / 7 / 24 \\
1 \mathrm{E} 4(\mathrm{p}) \\
\end{array}$ & 181511 & $1821 \quad 30$ & 182755 & .000 & -3.6 & 6.7 & & & & & & & & & \\
\hline ALMA-ATA & & $\begin{array}{cc}1821 & 29 \\
\pm 21\end{array}$ & & $\begin{array}{c}.247 \\
\pm .038\end{array}$ & & & 1 & Т 60. & CCDST & 26 & -27 & 0 & 2 & 0.5 & - \\
\hline $\begin{array}{r}97 / 7 / 25 \\
1 \mathrm{E} 4(\mathrm{~A}) \\
\end{array}$ & 20450 & $2052 \quad 2$ & 205858 & .300 & -3.6 & 4.2 & & & & & & & & & \\
\hline $\begin{array}{c}\text { ALMA-ATA } \\
\text { OHP }\end{array}$ & & $\begin{array}{cc}2051 & 50 \\
& \pm 20 \\
2051 & 37 \\
& \pm 13 \\
2051 & 27 \\
& \pm 43 \\
\end{array}$ & & $\begin{array}{c}.396 \\
\pm .059 \\
.470 \\
\pm .039 \\
.431 \\
\pm .120 \\
\end{array}$ & & & 25 & $\begin{array}{l}\text { Т } 60 . \\
\text { Т } 60 . \\
\text { Т } 80 .\end{array}$ & $\begin{array}{l}\text { PMW } \\
\text { CCDST } \\
\text { CCD1 }\end{array}$ & $\begin{array}{l}23 \\
30 \\
9\end{array}$ & $\begin{array}{l}-24 \\
-22 \\
-15\end{array}$ & 0 & $\begin{array}{c}2 V \\
2 \\
2\end{array}$ & $\begin{array}{c}- \\
0.5\end{array}$ & 35. \\
\hline $\begin{array}{l}97 / 7 / 25 \\
3 \mathrm{E} 2(\mathbf{P}) \\
\end{array}$ & 222555 & $2231 \quad 4$ & 223725 & .330 & -2.5 & 8.9 & & & & & & & & & \\
\hline $\begin{array}{c}\text { SEVILLA } \\
\text { CATANIA } \\
\text { ALMA-ATA } \\
\text { OCA } \\
\text { OCA } \\
\text { OCA } \\
\text { BUCHAREST1 } \\
\text { LISBOA } \\
\text { OHP }\end{array}$ & & $\begin{array}{cc}2232 & 43 \\
& \pm 10 \\
2232 & 3 \\
& \pm 29 \\
2232 & 0 \\
& \pm 36 \\
2232 & 17 \\
& \pm 22 \\
2232 & 7 \\
& \pm 19 \\
2232 & 24 \\
& \pm 23 \\
2231 & 59 \\
& \pm 23 \\
2231 & 48 \\
& \pm 69 \\
2232 & 18 \\
\pm 15 \\
\end{array}$ & & $\begin{array}{c}.806 \\
\pm .050 \\
.036 \\
\pm .006 \\
.321 \\
\pm .090 \\
.181 \\
\pm .063 \\
.543 \\
\pm .141 \\
.184 \\
.071 \\
.302 \\
\pm .049 \\
.319 \\
\pm .253 \\
.453 \\
\pm .044 \\
\end{array}$ & & & -38 & $\begin{array}{l}\text { Т } 25 . \\
\text { Т } 80 .\end{array}$ & $\begin{array}{l}\text { PM7 } \\
\text { PM7 } \\
\text { CCDI } \\
\text { CCD2 } \\
\text { CCD1 }\end{array}$ & $\begin{array}{l}19 \\
22\end{array}$ & $\begin{array}{l}-26 \\
-24 \\
-25\end{array}$ & $\begin{array}{l}0 \\
0\end{array}$ & $\begin{array}{c}B \\
R \\
V \\
1 G G \\
- \\
2\end{array}$ & $\begin{array}{c}- \\
0.1 \\
0.1 \\
0.5 \\
0.2 \\
0.2 \\
0.2\end{array}$ & $\begin{array}{l}- \\
- \\
- \\
- \\
-\end{array}$ \\
\hline $\begin{array}{l}97 / 7 / 25 \\
302(P) \\
\end{array}$ & 234418 & $2349 \quad 56$ & 235536 & .228 & 2.9 & 9.2 & & & & & & & & & \\
\hline $\begin{array}{c}\text { LUMEZZANE } \\
\text { NAUCHNY } \\
\text { OCA } \\
\text { OCA } \\
\text { OCA } \\
\text { SAN-FERN. } \\
\text { BOSKOOP } \\
\text { LISBOA } \\
\text { OHP }\end{array}$ & & $\begin{array}{cc}2350 & 23 \\
& \pm 20 \\
2350 & 6 \\
& \pm 14 \\
2350 & 42 \\
& \pm 14 \\
2351 & 10 \\
& \pm 16 \\
2350 & 49 \\
& \pm 17 \\
2350 & 13 \\
& \pm 50 \\
2350 & 7 \\
& \pm 53 \\
2350 & 11 \\
& \pm 31 \\
2351 & 29 \\
& \pm 7 \\
\end{array}$ & & $\begin{array}{c}.484 \\
\pm .060 \\
.459 \\
\pm .039 \\
.651 \\
\pm .055 \\
1.032 \\
.203 \\
.643 \\
\pm .060 \\
.476 \\
\pm .163 \\
.341 \\
\pm .108 \\
.441 \\
\pm .066 \\
.466 \\
\pm .019 \\
\end{array}$ & & & $\begin{array}{l}-11 \\
-15 \\
-93\end{array}$ & $\begin{array}{l}\text { Т } 30 . \\
\text { T } 25 . \\
\text { Т } 80 .\end{array}$ & $\begin{array}{c}\text { PM4 } \\
\text { CCDVX } \\
\text { CCD2 } \\
\text { CCD1 }\end{array}$ & $\begin{array}{l}21 \\
29 \\
29\end{array}$ & $\begin{array}{l}-18 \\
-31 \\
-27\end{array}$ & $\begin{array}{l}0 \\
0 \\
0\end{array}$ & $\begin{array}{l}- \\
- \\
- \\
1\end{array}$ & $\begin{array}{c}0.01 \\
- \\
0.2 \\
0.2 \\
0.2\end{array}$ & 35. \\
\hline $\begin{array}{l}97 / 7 / 26 \\
3 \mathrm{E} 4(\mathrm{p}) \\
\end{array}$ & 14740 & $152 \quad 51$ & 15759 & .067 & -2.3 & 6.2 & & & & & & & & & \\
\hline CATANIA & & $\begin{array}{rr}154 \quad 17 \\
\quad \pm 271 \\
\end{array}$ & & $\begin{array}{c}.002 \\
\pm .004 \\
\end{array}$ & & & -86 & Т 91. & PM & 31 & -21 & 0 & $1 \mathrm{~V}$ & 0.1 & 21. \\
\hline $\begin{array}{r}97 / 7 / 26 \\
301(P) \\
\end{array}$ & 232033 & $2323 \quad 21$ & 23268 & .296 & 1.3 & 3.0 & & & & & & & & & \\
\hline $\begin{array}{c}\text { CATANIA } \\
\text { OCA } \\
\text { OCA } \\
\text { OCA } \\
\text { SAN-FERN. }\end{array}$ & & $\begin{array}{cc}2323 & 17 \\
& \pm 5 \\
2324 & 19 \\
& \pm 15 \\
2324 & 18 \\
& \pm 6 \\
2324 & 13 \\
& \pm 7 \\
2323 & 16 \\
& \pm 30\end{array}$ & & $\begin{array}{c}.038 \\
\pm .002 \\
.558 \\
\pm .119 \\
.691 \\
\pm .132 \\
.749 \\
\pm .112 \\
.438 \\
\pm .166 \\
\end{array}$ & & & $\begin{array}{l}-58 \\
-57\end{array}$ & Т 150. & $\begin{array}{l}\text { PM7 } \\
\text { PM7 } \\
\text { PM7 } \\
\text { PM4 }\end{array}$ & $\begin{array}{l}35 \\
28\end{array}$ & $\begin{array}{l}-33 \\
-27 \\
-27 \\
-27\end{array}$ & 0 & $\begin{array}{c}2 V \\
B \\
R \\
V\end{array}$ & $\begin{array}{l}0.1 \\
0.2 \\
0.2 \\
0.2\end{array}$ & $\begin{array}{l}- \\
- \\
-\end{array}$ \\
\hline
\end{tabular}


J.-E. Arlot et al.: The PHEMU97 Campaign, Online Material p 6

Table 4. continued.

\begin{tabular}{|c|c|c|c|c|c|c|c|c|c|c|c|c|c|c|c|}
\hline $\begin{array}{c}\text { Dates } \\
\text { Phenomena } \\
\text { Locations } \\
\text { (1) }\end{array}$ & $\begin{array}{c}\text { Begins } \\
\mathrm{h} \mathrm{m} \mathrm{s} \\
\text { (2) }\end{array}$ & $\begin{array}{c}\underset{\text { M m }}{\text { Maxi. }} \\
\text { (3) }\end{array}$ & $\begin{array}{c}\text { Ends } \\
\mathrm{h} \mathrm{m} \mathrm{s} \\
\text { (4) }\end{array}$ & $\begin{array}{c}\text { Magn. } \\
\text { drop } \\
(5)\end{array}$ & $\begin{array}{l}\text { Ph. } \\
\text { (s) } \\
\text { (6) }\end{array}$ & $\begin{array}{l}\text { Dist. } \\
(\mathbf{R j})\end{array}$ & $\begin{array}{c}\text { C-O } \\
(\mathrm{s}) \\
(8)\end{array}$ & $\begin{array}{c}\text { Ap. } \\
\text { (cm) } \\
(9)\end{array}$ & $\begin{array}{l}\text { Rec. } \\
\text { (10) }\end{array}$ & $\begin{array}{l}\text { El. } \\
\text { Jup. } \\
\left({ }^{\circ}\right) \\
(11)\end{array}$ & $\begin{array}{l}\text { El. } \\
\text { Sun } \\
\left({ }^{\circ}\right) \\
(12)\end{array}$ & $\begin{array}{l}\text { Cd. } \\
\text { (13) }\end{array}$ & $\begin{array}{l}\text { Filt. } \\
\text { (14) }\end{array}$ & $\begin{array}{l}\text { T. } \\
\text { int. } \\
\text { (s) } \\
\text { (15) }\end{array}$ & $\begin{array}{l}\text { Dia. } \\
\text { (") } \\
\text { (16) }\end{array}$ \\
\hline OHP & & $\begin{array}{|cc|}2323 & 9 \\
& \pm 4 \\
\end{array}$ & & $\begin{array}{r}.533 \\
\pm .027 \\
\end{array}$ & & & 12 & $\mathrm{~T} 80$. & CCD1 & 27 & -27 & 0 & 1 & & \\
\hline $\begin{array}{l}97 / 7 / 27 \\
2 \mathrm{E1}(\mathrm{p}) \\
\end{array}$ & 11026 & $112 \quad 3$ & $\begin{array}{ll}1 & 1341 \\
\end{array}$ & . 050 & -.8 & 1.5 & & & & & & & & & \\
\hline SEVILLA & & \begin{tabular}{ll|}
11 & 40 \\
& \pm 18 \\
\end{tabular} & & $\begin{array}{c}.194 \\
\pm .022 \\
\end{array}$ & & & -37 & T 21. & VISU & 36 & -32 & 0 & 1 & & - \\
\hline $\begin{array}{l}97 / 7 / 29 \\
1 \mathrm{E3}(\mathrm{p})\end{array}$ & $\begin{array}{lll}23 & 8 & 44\end{array}$ & $2311 \quad 26$ & 23147 & .035 & -1.1 & 4.4 & & & & & & & & & \\
\hline $\begin{array}{l}\text { TENERIFE } \\
\text { SEVILLA }\end{array}$ & & $\begin{array}{cc}2311 & 39 \\
& \pm 21 \\
2310 & 55 \\
& \pm 3\end{array}$ & & $\begin{array}{c}.034 \\
\pm .008 \\
.327 \\
\pm .012\end{array}$ & & & $\begin{array}{l}-13 \\
31\end{array}$ & $\begin{array}{l}\text { Т } 150 . \\
\text { т } 21 .\end{array}$ & $\begin{array}{l}\text { PMIR } \\
\text { VISU }\end{array}$ & $\begin{array}{l}30 \\
29\end{array}$ & $\begin{array}{l}-35 \\
-31\end{array}$ & $\begin{array}{l}0 \\
0\end{array}$ & $\begin{array}{c}K \\
1\end{array}$ & 2.5 & 20. \\
\hline $\begin{array}{l}97 / 7 / 29 \\
103(P) \\
\end{array}$ & 234647 & $2349 \quad 40$ & 235234 & .206 & 1.8 & 4.1 & & & & & & & & & \\
\hline $\begin{array}{c}\text { TENERIFE } \\
\text { CATANIA } \\
\text { BUCHAREST1 } \\
\text { BOSKOOP } \\
\text { REUX } \\
\text { COMTHUREY } \\
\text { OHP }\end{array}$ & & $\begin{array}{cc}2349 & 58 \\
& \pm 21 \\
2349 & 24 \\
& \pm 11 \\
2349 & 41 \\
& \pm 19 \\
2349 & 23 \\
& \pm 41 \\
2350 & 2 \\
& \pm 48 \\
2349 & 24 \\
& \pm 62 \\
2349 & 42 \\
& \pm 14\end{array}$ & & $\begin{array}{l}.118 \\
\pm .026 \\
.016 \\
\pm .002 \\
.231 \\
\pm .055 \\
.112 \\
\pm .065 \\
.336 \\
\pm .144 \\
.217 \\
\pm .131 \\
.193 \\
\pm .032\end{array}$ & & & $\begin{array}{c}-22 \\
16\end{array}$ & $\begin{array}{l}\text { T } 30 . \\
\text { T } 30 . \\
\text { L } 18 . \\
\text { T } 80 .\end{array}$ & $\begin{array}{c}\text { PMIR } \\
\text { PM } \\
\text { CCDI } \\
\text { CCDVX } \\
\text { CCD5 } \\
\text { CCD6 } \\
\text { CCD1 }\end{array}$ & $\begin{array}{l}29 \\
21 \\
23 \\
20 \\
29\end{array}$ & $\begin{array}{l}-33 \\
-24 \\
-19 \\
-21 \\
-18 \\
-28\end{array}$ & $\begin{array}{l}0 \\
0 \\
0 \\
0 \\
0 \\
0\end{array}$ & $\begin{array}{c}2 V \\
3 G G \\
- \\
- \\
2\end{array}$ & $\begin{array}{l}2.5 \\
0.1 \\
0.5 \\
0.04 \\
0.04 \\
0.04 \\
-\end{array}$ & $\begin{array}{l}- \\
- \\
-\end{array}$ \\
\hline $\begin{array}{c}97 / 8 / 1 \\
4 \mathrm{E} 3(\mathrm{~A}) \\
\end{array}$ & $\begin{array}{lll}0 & 5 & 56 \\
\end{array}$ & $020 \quad 50$ & 03552 & .601 & -4.7 & 14. & & & & & & & & & \\
\hline $\begin{array}{c}\text { BARCELONA } \\
\text { TENERIFE } \\
\text { SEVILLA } \\
\text { CATANIA } \\
\text { SAN-FERN. } \\
\text { LISBOA } \\
\text { OHP }\end{array}$ & & 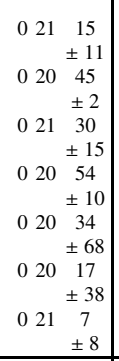 & & $\begin{array}{c}6.516 \\
\pm .495 \\
.926 \\
\pm .006 \\
2.210 \\
\pm .146 \\
.079 \\
\pm .003 \\
.163 \\
\pm .280 \\
.936 \\
\pm .124 \\
.947 \\
\pm .025 \\
\end{array}$ & & & $\begin{array}{c}5 \\
-40\end{array}$ & $\begin{array}{l}\text { T } 16 . \\
\text { T } 150 . \\
\text { T } 21 . \\
\text { T } 91 . \\
\text { T } 33 . \\
\text { T } 25 . \\
\text { T } 80 .\end{array}$ & $\begin{array}{c}\text { VISU } \\
\text { PMIR } \\
\text { VISU } \\
\text { PM } \\
\text { PM4 } \\
\text { CCD2 } \\
\text { CCD1 }\end{array}$ & $\begin{array}{l}32 \\
40 \\
35\end{array}$ & $\begin{array}{l}-30 \\
-42 \\
-35 \\
-31 \\
-35 \\
-33 \\
-27\end{array}$ & $\begin{array}{l}0 \\
0 \\
0 \\
0 \\
0 \\
0\end{array}$ & $\begin{array}{c}2 \\
K \\
1 \\
2 V \\
- \\
-\end{array}$ & $\begin{array}{l}4 . \\
- \\
0.1\end{array}$ & $\begin{array}{c}- \\
20 . \\
- \\
21 .\end{array}$ \\
\hline $\begin{array}{l}97 / 8 / 1 \\
4 \mathrm{E} 2(\mathrm{p}) \\
\end{array}$ & $\begin{array}{lll}20 & 624 \\
\end{array}$ & $2011 \quad 34$ & 201642 & .191 & -1.1 & 7.5 & & & & & & & & & \\
\hline KAVALUR & & $\begin{array}{rr}2011 \quad 49 \\
\quad \pm 3 \\
\end{array}$ & & $\begin{array}{c}.193 \\
\pm .004 \\
\end{array}$ & & & -15 & Т 235. & $\mathrm{CCDC}$ & 59 & -54 & 0 & $1 * 3$ & 4. & - \\
\hline $\begin{array}{r}97 / 8 / 2 \\
3 \mathrm{E} 2(\mathrm{P}) \\
\end{array}$ & $\begin{array}{lll}2 & 4 & 40 \\
\end{array}$ & $211 \quad 6$ & 21731 & .393 & -1.5 & 9.2 & & & & & & & & & \\
\hline $\begin{array}{l}\text { SEVILLA } \\
\text { TENERIFE } \\
\text { CATANIA }\end{array}$ & & $\begin{array}{ccc}2 & 13 & 46 \\
& \pm 0 \\
2 & 12 & 2 \\
& & \pm 37 \\
& 11 & 9 \\
& 11 & \\
& \pm 27\end{array}$ & & $\begin{array}{c}.541 \\
\pm .001 \\
.012 \\
\pm .005 \\
.046 \\
\pm .010\end{array}$ & & & $\begin{array}{c}-161 \\
-57 \\
-4\end{array}$ & $\begin{array}{l}\text { T } 21 . \\
\text { T } 150 . \\
\text { T } 91 .\end{array}$ & $\begin{array}{l}\text { VISU } \\
\text { PMIR }\end{array}$ & 25 & $\begin{array}{l}-30 \\
-42 \\
-19\end{array}$ & 0 & $\begin{array}{c}K \\
2 V\end{array}$ & 0.1 & 21. \\
\hline $\begin{array}{r}97 / 8 / 2 \\
302(T) \\
\end{array}$ & 24644 & $253 \quad 4$ & 25927 & .259 & 1.6 & 9.3 & & & & & & & & & \\
\hline $\begin{array}{c}\text { TENERIFE } \\
\text { OCA } \\
\text { OCA } \\
\text { OCA } \\
\text { SAN-FERN. } \\
\text { CATANIA } \\
\text { OHP }\end{array}$ & & $\begin{array}{ccc}253 & 3 \\
& \pm 24 \\
253 & 5 \\
& 523 \\
& \pm 21 \\
251 & 27 \\
& \pm 37 \\
252 & 55 \\
& \pm 27 \\
254 & 30 \\
& \pm 178 \\
253 & 14 \\
& \pm 37 \\
253 & 20 \\
& \pm 14 \\
\end{array}$ & & $\begin{array}{c}.330 \\
\pm .042 \\
.608 \\
\pm .056 \\
.452 \\
\pm .232 \\
.600 \\
\pm .084 \\
.164 \\
\pm .077 \\
.590 \\
\pm .102 \\
.512 \\
\pm .042 \\
\end{array}$ & & & $\begin{array}{c}9 \\
-86 \\
-10 \\
-16\end{array}$ & $\begin{array}{l}\text { T } 150 . \\
\text { T } 150 . \\
\text { T } 150 . \\
\text { T } 150 .\end{array}$ & $\begin{array}{l}\text { PMIR } \\
\text { PM7 } \\
\text { PM7 } \\
\text { PM7 }\end{array}$ & $\begin{array}{l}42 \\
19\end{array}$ & $\begin{array}{l}-38 \\
-14 \\
-24 \\
-24 \\
-26 \\
-13 \\
-14\end{array}$ & $\begin{array}{l}0 \\
0 \\
0 \\
0 \\
0 \\
0 \\
0\end{array}$ & $\begin{array}{l}K \\
B \\
R \\
V\end{array}$ & $\begin{array}{c}4 . \\
0.2 \\
0.2 \\
0.2\end{array}$ & 28. \\
\hline $\begin{array}{r}97 / 8 / 3 \\
4 \mathrm{E} 1(\mathrm{~A}) \\
\end{array}$ & $\begin{array}{lll}0 & 0 & 17 \\
\end{array}$ & $\begin{array}{lll}0 & 5 & 3 \\
\end{array}$ & $\begin{array}{lll}0 & 9 & 48\end{array}$ & .770 & -.8 & 3.7 & & & & & & & & & \\
\hline TENERIFE & & $\begin{array}{ccc}0 & 5 & 2 \\
& \pm 1\end{array}$ & & $\begin{array}{r}1.556 \\
\pm .013\end{array}$ & & & 1 & T 150. & PMIR & 40 & -41 & 0 & K & 4. & 20. \\
\hline
\end{tabular}


J.-E. Arlot et al.: The PHEMU97 Campaign, Online Material $p 7$

Table 4. continued.

\begin{tabular}{|c|c|c|c|c|c|c|c|c|c|c|c|c|c|c|c|}
\hline $\begin{array}{c}\text { Dates } \\
\text { Phenomena } \\
\text { Locations } \\
\text { (1) }\end{array}$ & $\begin{array}{c}\text { Begins } \\
\mathrm{h} \mathrm{m} \mathrm{s} \\
\text { (2) }\end{array}$ & $\begin{array}{c}\text { Maxi. } \\
\mathrm{h} \mathrm{m} \\
\text { (3) }\end{array}$ & $\begin{array}{c}\begin{array}{c}\text { Ends } \\
\text { h m s }\end{array} \\
\text { (4) }\end{array}$ & $\begin{array}{c}\text { Magn. } \\
\text { drop } \\
(5)\end{array}$ & $\begin{array}{l}\text { Ph. } \\
\text { (s) } \\
\text { (6) }\end{array}$ & $\begin{array}{l}\text { Dist. } \\
\text { (Rj) }\end{array}$ & $\begin{array}{l}\mathrm{C}-\mathrm{O} \\
(\mathrm{s})\end{array}$ & $\begin{array}{c}\text { Ap. } \\
(\mathbf{c m})\end{array}$ & (10) & $\begin{array}{c}\text { El. } \\
\text { Jup. } \\
\left({ }^{\circ}\right) \\
(11)\end{array}$ & $\begin{array}{l}\text { El. } \\
\text { Sun } \\
\left({ }^{\circ}\right) \\
(\mathbf{1 2})\end{array}$ & $\begin{array}{l}\text { Cd. } \\
\text { (13) }\end{array}$ & (14) & $\begin{array}{l}\text { T. } \\
\text { int. } \\
\text { (s) } \\
\text { (15) }\end{array}$ & $\begin{array}{l}\text { Dia. } \\
\text { (") } \\
\text { (16) }\end{array}$ \\
\hline PRAHA & & 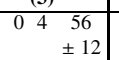 & & $\begin{array}{c}901 \\
\pm .107\end{array}$ & & & $\frac{101}{7}$ & T30. & $\mathrm{V1}$ & 23 & $\frac{1121}{-21}$ & $\frac{(10)}{0}$ & 2 & 0.02 & - \\
\hline CATANIA2 & & $\begin{array}{ccc}0 & 5 & 9 \\
& & \pm 6\end{array}$ & & $\begin{array}{l}1.410 \\
\pm .080\end{array}$ & & & -6 & Т 20. & VISU & 35 & -33 & 0 & 2 & - & - \\
\hline CATANIA & & $\begin{array}{ccc}0 & 5 & 3 \\
& & \pm 7\end{array}$ & & $\begin{array}{c}.528 \\
\pm .079\end{array}$ & & & 0 & Т 91. & PM & 35 & -33 & 0 & $2 V$ & 0.1 & 21. \\
\hline NAUCHNY & & $\begin{array}{ccc}0 & 5 & 3 \\
+4\end{array} \mid$ & & $\begin{array}{l}1.414 \\
+066\end{array}$ & & & 0 & T 60. & PMW & 24 & -20 & 0 & V & - & 35. \\
\hline $\mathrm{OCA}$ & & $\begin{array}{lll}0 & 4 & 56\end{array}$ & & 1.163 & & & 7 & Т 150. & PM7 & 30 & -28 & 0 & $B$ & 0.2 & - \\
\hline OCA & & $\left|\begin{array}{ccc}0 & 5 & 7 \\
& & 7 \\
& \pm 5\end{array}\right|$ & & $\begin{array}{l}1.457 \\
\pm .084\end{array}$ & & & -4 & Т 150. & PM7 & 30 & -28 & 0 & $R$ & 0.2 & - \\
\hline OCA & & $\begin{array}{ccc}0 & 5 & 1 \\
09\end{array}$ & & $\begin{array}{l}1.475 \\
\pm .166\end{array}$ & & & 2 & Т 150. & PM7 & 30 & -28 & 0 & V & 0.2 & - \\
\hline COMTHUREY & & \begin{tabular}{ccc|}
0 & 5 & 5 \\
& & \pm 23
\end{tabular} & & $\begin{array}{r}1.911 \\
\pm .404 \\
\end{array}$ & & & -2 & L 18. & CCD6 & 20 & -18 & 0 & 2 & 0.04 & - \\
\hline $\begin{array}{r}97 / 8 / 3 \\
301(P) \\
\end{array}$ & 14220 & $145 \quad 4$ & 14748 & .311 & .6 & 2.5 & & & & & & & & & \\
\hline BOSKOOP & & $\begin{array}{rlr}144 & 54 \\
& \pm 28 \\
\end{array}$ & & $\begin{array}{c}.273 \\
\pm .094 \\
\end{array}$ & & & 10 & Т 30. & CCDVX & 19 & -16 & 0 & - & - & - \\
\hline $\begin{array}{r}97 / 8 / 6 \\
103(P) \\
\end{array}$ & 21414 & $217 \quad 26$ & 22040 & .245 & .7 & 4.5 & & & & & & & & & \\
\hline TENERIFE & & $\begin{array}{lll}217 & 29 \\
& \pm 2\end{array}$ & & $\begin{array}{l}.169 \\
\pm .004\end{array}$ & & & -3 & Т 150. & PMIR & 44 & -42 & 0 & K & 4. & 20. \\
\hline OCA & & $\begin{array}{ccc}217 & 42 \\
& \pm 12\end{array}$ & & $\begin{array}{c}.332 \\
\pm .049\end{array}$ & & & -16 & Т 150. & PM7 & 21 & -19 & 0 & B & 0.2 & - \\
\hline OCA & & $\begin{array}{ccc}216 & 36 \\
& \pm 71\end{array}$ & & $\begin{array}{l}.184 \\
\pm .050\end{array}$ & & & 50 & Т 150. & PM7 & 21 & -19 & 0 & $R$ & 0.2 & - \\
\hline OCA & & $\begin{array}{ccc}217 & 35 \\
& \pm 20\end{array}$ & & $\begin{array}{c}.412 \\
\pm .077\end{array}$ & & & -9 & Т 150. & PM7 & 21 & -19 & 0 & V & 0.2 & - \\
\hline MUNICH & & \begin{tabular}{cc|}
217 & 13 \\
& \pm 31 \\
\end{tabular} & & $\begin{array}{c}.257 \\
\pm .118 \\
\end{array}$ & & & 13 & T 28. & CCDM & 16 & -14 & 0 & $2 * 4$ & - & - \\
\hline $\begin{array}{l}97 / 8 / 10 \\
301(\mathrm{~T}) \\
\end{array}$ & $\begin{array}{lll}4 & 2 & 52 \\
\end{array}$ & $\begin{array}{l}45 \quad 34 \\
\end{array}$ & $\begin{array}{llll}4 & 8 & 16\end{array}$ & .320 & -.1 & 2.0 & & & & & & & & & \\
\hline TENERIFE & & $\begin{array}{lll}45 & 26 \\
& \pm 1\end{array}$ & & $\begin{array}{l}1.002 \\
\pm .018\end{array}$ & & & 8 & Т 150. & PMIR & 28 & -29 & 0 & K & 4. & 20. \\
\hline $\begin{array}{l}97 / 8 / 10 \\
3 \mathrm{E} 4(\mathrm{~A}) \\
\end{array}$ & $\begin{array}{lll}11 & 9 & 58 \\
\end{array}$ & $1116 \quad 55$ & 112352 & .597 & .3 & 5.6 & & & & & & & & & \\
\hline ELLINBANK & & \begin{tabular}{cc|}
1116 & 48 \\
& \pm 12 \\
\end{tabular} & & $\begin{array}{c}.188 \\
\pm .018 \\
\end{array}$ & & & 7 & Т 32. & $\mathrm{CCD} 2$ & 21 & -21 & 0 & $2 \mathrm{~V}$ & 2. & 25. \\
\hline $\begin{array}{l}97 / 8 / 13 \\
103(P) \\
\end{array}$ & $\begin{array}{lll}444 \quad 2 \\
\end{array}$ & $447 \quad 38$ & 45115 & .291 & -.8 & 4.9 & & & & & & & & & \\
\hline TOPEKA & & $\begin{array}{ccc}447 & 42 \\
& \pm 69\end{array}$ & & $\begin{array}{c}.056 \\
\pm .023\end{array}$ & & & 4 & L 38. & $\mathrm{CCD} 12$ & 31 & -32 & 0 & 1 & & - \\
\hline $\begin{array}{l}97 / 8 / 23 \\
302(P) \\
\end{array}$ & 12217 & $1229 \quad 46$ & 123832 & .259 & -4.1 & 9.3 & & & & & & & & & \\
\hline OKAYAMA & & $\begin{array}{rr}1229 & 56 \\
\pm 25 \\
\end{array}$ & & $\begin{array}{c}.495 \\
\pm .045 \\
\end{array}$ & & & -10 & Т 35. & PM3 & 33 & -31 & 0 & $2 \mathrm{~V}$ & 0.2 & 40. \\
\hline $\begin{array}{l}97 / 8 / 23 \\
3 \mathrm{E} 2(\mathrm{P}) \\
\end{array}$ & 135432 & $143 \quad 34$ & 141245 & .284 & 4.0 & 9.2 & & & & & & & & & \\
\hline KAVALUR & & 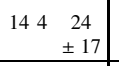 & & $\begin{array}{c}.314 \\
\pm .022 \\
\end{array}$ & & & 50 & Т 102. & $\mathrm{CCDC}$ & 27 & -16 & 0 & $1 * 3$ & - & - \\
\hline $\begin{array}{r}97 / 8 / 27 \\
1 \mathrm{E3}(\mathrm{A}) \\
\end{array}$ & 112240 & $1129 \quad 28$ & 113625 & .452 & 5.2 & 4.9 & & & & & & & & & \\
\hline $\begin{array}{l}\text { OKAYAMA } \\
\text { FUNAHO }\end{array}$ & & $\begin{array}{rr}1129 & 17 \\
& \pm 27 \\
1129 & 20 \\
& \pm 31 \\
\end{array}$ & & $\begin{array}{c}.535 \\
\pm .100 \\
.449 \\
\pm .095 \\
\end{array}$ & & & $\begin{array}{c}12 \\
9\end{array}$ & $\begin{array}{l}\text { T } 35 . \\
\text { т } 28 .\end{array}$ & $\begin{array}{l}\text { PM3 } \\
\text { PM3 }\end{array}$ & $\begin{array}{l}28 \\
28\end{array}$ & $\begin{array}{l}-22 \\
-22\end{array}$ & $\begin{array}{l}0 \\
0\end{array}$ & $\begin{array}{l}1 V \\
2 V\end{array}$ & $\begin{array}{l}0.2 \\
0.2\end{array}$ & $\begin{array}{l}40 . \\
-\end{array}$ \\
\hline $\begin{array}{l}97 / 8 / 28 \\
1 \mathrm{E3}(\mathrm{P}) \\
\end{array}$ & $\begin{array}{lll}025 & 0 \\
\end{array}$ & $036 \quad 22$ & 04755 & .199 & -9.8 & 2.2 & & & & & & & & & \\
\hline $\begin{array}{l}\text { TENERIFE } \\
\text { CATANIA }\end{array}$ & & $\begin{array}{ccc}036 & 24 \\
& \pm 10 \\
0 & 36 & 45 \\
& \pm 99 \\
\end{array}$ & & $\begin{array}{c}.233 \\
\pm .006 \\
.021 \\
\pm .006 \\
\end{array}$ & & & $\begin{array}{l}-2 \\
-23\end{array}$ & $\begin{array}{l}\text { T } 150 . \\
\text { Т } 91 .\end{array}$ & $\begin{array}{l}\text { PMIR } \\
\text { PM }\end{array}$ & $\begin{array}{l}43 \\
22\end{array}$ & $\begin{array}{l}-51 \\
-37\end{array}$ & $\begin{array}{l}0 \\
0\end{array}$ & $\begin{array}{c}K \\
1 V\end{array}$ & $\begin{array}{l}4 . \\
0.1\end{array}$ & 20. \\
\hline $\begin{array}{l}97 / 8 / 28 \\
1 \mathrm{E} 4(\mathrm{p}) \\
\end{array}$ & 2417 & $252 \quad 10$ & $\begin{array}{llll}3 & 3 & 39 \\
\end{array}$ & .128 & -7.5 & 4.8 & & & & & & & & & \\
\hline TENERIFE & & \begin{tabular}{cc|}
252 & 40 \\
& \pm 25 \\
\end{tabular} & & $\begin{array}{c}.106 \\
\pm .012 \\
\end{array}$ & & & -30 & Т 150. & PMIR & 26 & -44 & 0 & K & 4. & 20. \\
\hline $\begin{array}{l}97 / 8 / 30 \\
302(P)\end{array}$ & 155352 & $\begin{array}{ll}163 & 17\end{array}$ & 161253 & .184 & -7.7 & 9.2 & & & & & & & & & \\
\hline
\end{tabular}


J.-E. Arlot et al.: The PHEMU97 Campaign, Online Material $p 8$

Table 4. continued.

\begin{tabular}{|c|c|c|c|c|c|c|c|c|c|c|c|c|c|c|c|}
\hline $\begin{array}{c}\text { Dates } \\
\text { Phenomena } \\
\text { Locations } \\
\text { (1) }\end{array}$ & $\begin{array}{c}\text { Begins } \\
\text { h m s } \\
(2)\end{array}$ & $\begin{array}{c}\text { Maxi. } \\
\text { h m s } \\
\text { (3) }\end{array}$ & $\begin{array}{l}\text { Ends } \\
\text { h m s } \\
\text { (4) }\end{array}$ & $\begin{array}{c}\text { Magn. } \\
\text { drop } \\
(5)\end{array}$ & $\begin{array}{l}\text { Ph. } \\
(\mathrm{s}) \\
(6)\end{array}$ & $\begin{array}{l}\text { Dist. } \\
(\mathbf{R j}) \\
(7)\end{array}$ & $\begin{array}{c}\text { C-O } \\
(s) \\
(8)\end{array}$ & $\begin{array}{c}\begin{array}{c}\text { Ap. } \\
\text { (cm) }\end{array} \\
(9) \\
\end{array}$ & $\begin{array}{l}\text { Rec. } \\
\text { (10) }\end{array}$ & $\begin{array}{c}\text { El. } \\
\text { Jup. } \\
\left(^{\circ}\right) \\
(11)\end{array}$ & $\begin{array}{l}\text { El. } \\
\text { Sun } \\
\left({ }^{\circ}\right) \\
(12)\end{array}$ & $\begin{array}{l}\text { Cd. } \\
\text { (13) }\end{array}$ & $\begin{array}{l}\text { Filt. } \\
\text { (14) }\end{array}$ & $\begin{array}{l}\text { T. } \\
\text { int. } \\
\text { (s) } \\
(15)\end{array}$ & $\begin{array}{c}\text { Dia. } \\
\text { (") } \\
\text { (16) }\end{array}$ \\
\hline $\begin{array}{l}\text { OKAYAMA } \\
\text { ALMA-ATA }\end{array}$ & & $\begin{array}{cc}164 & 59 \\
& \pm 63 \\
163 & 42 \\
& \pm 16 \\
\end{array}$ & & $\begin{array}{c}.449 \\
\pm .104 \\
.309 \\
\pm .015 \\
\end{array}$ & & & $\begin{array}{l}-69 \\
-25\end{array}$ & $\begin{array}{l}\text { Т } 35 . \\
\text { Т } 60 .\end{array}$ & $\begin{array}{c}\text { PM3 } \\
\text { CCDST }\end{array}$ & $\begin{array}{l}28 \\
27\end{array}$ & $\begin{array}{l}-44 \\
-26\end{array}$ & 0 & $\begin{array}{l}3 V \\
2 R\end{array}$ & $\begin{array}{l}0.2 \\
2 .\end{array}$ & 40. \\
\hline $\begin{array}{l}\text { 97/ 8/30 } \\
3 \text { E2( p) } \\
\end{array}$ & 183454 & $1845 \quad 10$ & 185541 & .111 & 6.5 & 8.8 & & & & & & & & & \\
\hline $\begin{array}{c}\text { OHP } \\
\text { ALMA-ATA } \\
\text { KAVALUR }\end{array}$ & & $\begin{array}{rr}1845 & 53 \\
& \pm 150 \\
1844 & 40 \\
\pm 112 \\
1842 & 7 \\
\pm 34 \\
\pm\end{array}$ & & $\begin{array}{c}.099 \\
\pm .064 \\
.074 \\
\pm .041 \\
.239 \\
\pm .030 \\
\end{array}$ & & & $\begin{array}{l}-43 \\
30 \\
183\end{array}$ & $\begin{array}{l}\text { T } 80 . \\
\text { T } 60 . \\
\text { T } 102 .\end{array}$ & $\begin{array}{l}\text { CCD1 } \\
\text { CCDST } \\
\text { CCDC }\end{array}$ & $\begin{array}{l}13 \\
26 \\
53\end{array}$ & $\begin{array}{l}-6 \\
-38 \\
-69\end{array}$ & 0 & $\begin{array}{l}2 \mathrm{I} \\
2 R \\
2 * 3\end{array}$ & $\begin{array}{l}3 . \\
0.5 \\
-\end{array}$ & - \\
\hline $\begin{array}{r}97 / 9 / 3 \\
1 \mathrm{E3}(\mathrm{A}) \\
\end{array}$ & $1515 \quad 3$ & $\begin{array}{ll}1526 & 7 \\
\end{array}$ & 153750 & .486 & 12 & & & & & & & & & & \\
\hline ALMA-ATA & & $\begin{array}{rc}1525 & 41 \\
& \pm 14 \\
\end{array}$ & & $\begin{array}{c}.761 \\
\pm .034 \\
\end{array}$ & & & 26 & T 60. & CCDST & 25 & -22 & 0 & $2 R$ & 1. & - \\
\hline $\begin{array}{c}97 / 9 / 4 \\
1 \mathrm{E} 3(\mathrm{P}) \\
\end{array}$ & 1379 & $1313 \quad 13$ & 131913 & .298 & 6.5 & 7.0 & & & & & & & & & \\
\hline OKAYAMA & & $\begin{array}{rc}1313 & 5 \\
& \pm 23 \\
\end{array}$ & & $\begin{array}{c}.324 \\
\pm .055 \\
\end{array}$ & & & 8 & Т 35. & PM3 & 38 & -41 & 0 & $1 \mathrm{~V}$ & 0.2 & 40. \\
\hline $\begin{array}{c}97 / 9 / 8 \\
3 \mathrm{E} 2(\mathrm{P}) \\
\end{array}$ & 105260 & $114 \quad 5$ & 111458 & .999 & 8.3 & 9.3 & & & & & & & & & \\
\hline $\begin{array}{l}\text { FUNAHO } \\
\text { OKAYAMA }\end{array}$ & & 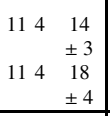 & & $\begin{array}{c}2.857 \\
\pm .043 \\
3.214 \\
\pm .049 \\
\end{array}$ & & & $\begin{array}{l}-9 \\
-13\end{array}$ & $\begin{array}{l}\text { Т } 28 . \\
\text { T } 35 .\end{array}$ & $\begin{array}{l}\text { PM3 } \\
\text { PM3 }\end{array}$ & $\begin{array}{l}31 \\
31\end{array}$ & $\begin{array}{l}-21 \\
-21\end{array}$ & $\begin{array}{l}0 \\
0\end{array}$ & $\begin{array}{l}1 \mathrm{~V} \\
2 \mathrm{~V}\end{array}$ & $\begin{array}{l}1 . \\
1 .\end{array}$ & - \\
\hline $\begin{array}{c}97 / 9 / 10 \\
103(\mathrm{~A}) \\
\end{array}$ & $\begin{array}{lll}16 & 8 & 49 \\
\end{array}$ & $1617 \quad 16$ & 16263 & .356 & -16.2 & 5.8 & & & & & & & & & \\
\hline $\begin{array}{c}\text { ALMA-ATA } \\
\text { FUNAHO } \\
\text { OKAYAMA }\end{array}$ & & $\begin{array}{rc}1617 & 28 \\
& \pm 21 \\
1617 & 37 \\
& \pm 27 \\
1617 & 38 \\
& \pm 27 \\
\end{array}$ & & $\begin{array}{c}.363 \\
\pm .028 \\
.314 \\
\pm .034 \\
.321 \\
\pm .039 \\
\end{array}$ & & & $\begin{array}{l}-12 \\
-21 \\
-22\end{array}$ & $\begin{array}{l}\text { T } 60 . \\
\text { T } 28 . \\
\text { T } 35 .\end{array}$ & $\begin{array}{c}\text { CCDST } \\
\text { PM3 } \\
\text { PM3 }\end{array}$ & $\begin{array}{l}29 \\
18 \\
18\end{array}$ & $\begin{array}{l}-32 \\
-47 \\
-47\end{array}$ & $\begin{array}{l}0 \\
0 \\
0\end{array}$ & $\begin{array}{c}R \\
2 V \\
1 V\end{array}$ & $\begin{array}{l}- \\
1 . \\
1 .\end{array}$ & $\begin{array}{l}- \\
- \\
40 .\end{array}$ \\
\hline $\begin{array}{l}97 / 9 / 11 \\
103(P) \\
\end{array}$ & $\begin{array}{lll}021 & 20 \\
\end{array}$ & $\begin{array}{lll}032 & 28 \\
\end{array}$ & 04324 & .204 & 17.7 & 1.4 & & & & & & & & & \\
\hline $\begin{array}{l}\text { CATANIA } \\
\text { BORDEAUX }\end{array}$ & & $\begin{array}{ccc}033 & 35 \\
& \quad \pm 159 \\
032 & 23 \\
& \quad \pm 178 \\
\end{array}$ & & $\begin{array}{c}.034 \\
\pm .012 \\
.240 \\
\pm .140 \\
\end{array}$ & & & $\begin{array}{c}-67 \\
5\end{array}$ & $\begin{array}{l}\text { Т } 91 . \\
\text { Т } 62 .\end{array}$ & $\begin{array}{c}\text { PM } \\
\text { CCD1 }\end{array}$ & $\begin{array}{l}13 \\
17\end{array}$ & $\begin{array}{l}-42 \\
-40\end{array}$ & $\begin{array}{l}0 \\
0\end{array}$ & $\begin{array}{c}2 \mathrm{~V} \\
2\end{array}$ & $\begin{array}{c}0.1 \\
1 .\end{array}$ & 28. \\
\hline $\begin{array}{l}97 / 9 / 11 \\
103(P) \\
\end{array}$ & 14350 & $148 \quad 15$ & 141236 & .124 & -6.9 & 5.8 & & & & & & & & & \\
\hline $\begin{array}{l}\text { OKAYAMA } \\
\text { FUNAHO }\end{array}$ & & $\begin{array}{ccc}149 & 13 \\
& \pm 13 \\
148 & 4 \\
& & \pm 17 \\
& & \pm 17 \\
\end{array}$ & & $\begin{array}{c}.232 \\
\pm .028 \\
.385 \\
\pm .049 \\
\end{array}$ & & & $\begin{array}{c}-58 \\
11\end{array}$ & $\begin{array}{l}\text { T } 35 . \\
\text { T } 28 .\end{array}$ & $\begin{array}{l}\text { PM3 } \\
\text { PM3 }\end{array}$ & $\begin{array}{l}34 \\
34\end{array}$ & $\begin{array}{l}-49 \\
-49\end{array}$ & $\begin{array}{l}0 \\
0\end{array}$ & $\begin{array}{l}3 V \\
2 V\end{array}$ & $\begin{array}{c}1 . \\
0.2\end{array}$ & 40. \\
\hline $\begin{array}{r}97 / 9 / 11 \\
1 \mathrm{E3}(\mathrm{A}) \\
\end{array}$ & 16256 & $1630 \quad 20$ & 163531 & .422 & 6.9 & 6.9 & & & & & & & & & \\
\hline KAVALUR & & $\begin{array}{cc}1630 & 2 \\
& \pm 2 \\
\end{array}$ & & $\begin{array}{c}.951 \\
\pm .020 \\
\end{array}$ & & & 18 & Т 102. & $\mathrm{CCDC}$ & 60 & -54 & 0 & $1 * 3$ & - & - \\
\hline $\begin{array}{l}\text { 97/9/15 } \\
3 \text { E2( P) } \\
\end{array}$ & $\begin{array}{lll}15 & 0 & 12 \\
\end{array}$ & $159 \quad 28$ & 151838 & .986 & 8.4 & 9.2 & & & & & & & & & \\
\hline ALMA-ATA & & $\begin{array}{rr}159 & 34 \\
& \pm 5 \\
\end{array}$ & & $\begin{array}{c}5.807 \\
\pm .250 \\
\end{array}$ & & & -6 & $\mathrm{~T} 60$. & CCDST & 27 & -24 & 0 & $2 R$ & 1. & - \\
\hline $\begin{array}{l}97 / 9 / 18 \\
1 \mathrm{E3}(\mathrm{A}) \\
\end{array}$ & 193010 & $1934 \quad 52$ & 193931 & .472 & 7.1 & 6.7 & & & & & & & & & \\
\hline LUMEZZANE & & $\begin{array}{rl}1934 & 57 \\
& \pm 8\end{array}$ & & $\begin{array}{c}.696 \\
\pm .062\end{array}$ & & & -5 & T 11. & VISU & 25 & -23 & 0 & 2 & - & 20. \\
\hline BARCELONA & & $\begin{array}{rl}1934 & 45 \\
& \pm 9\end{array}$ & & $\begin{array}{c}6.884 \\
\pm .610\end{array}$ & & & 7 & Т 16. & VISU & 27 & -19 & 0 & - & - & - \\
\hline MILANO & & $\begin{array}{cc}1935 & 36 \\
& \pm 0\end{array}$ & & $\begin{array}{c}.716 \\
\pm .003\end{array}$ & & & -44 & L 8. & VISU & 25 & -23 & 0 & 2 & - & - \\
\hline OCA & & $\begin{array}{rl}1934 & 39 \\
& \pm 6\end{array}$ & & $\begin{array}{c}.727 \\
\pm .044\end{array}$ & & & 13 & Т 150. & PM7 & 26 & -22 & 0 & $B$ & 0.2 & - \\
\hline OCA & & $\begin{array}{cc}1934 & 29 \\
& \pm 6\end{array}$ & & $\begin{array}{c}.678 \\
\pm .039\end{array}$ & & & 23 & Т 150. & PM7 & 26 & -22 & 0 & $R$ & 0.2 & - \\
\hline OCA & & $\begin{array}{r}1934 \quad 47 \\
\pm 13\end{array}$ & & $\begin{array}{l}.773 \\
\pm .111\end{array}$ & & & 5 & Т 150. & PM7 & 26 & -22 & 0 & $V$ & 0.2 & - \\
\hline NAUCHNY & & $\begin{array}{rc}1934 & 39 \\
& \pm 8\end{array}$ & & $\begin{array}{c}.651 \\
\pm .056\end{array}$ & & & 13 & Т 150. & PMW & 27 & -36 & 0 & $V$ & - & 35. \\
\hline PRAHA & & $\begin{array}{ll}1934 & 51\end{array}$ & & .689 & & & 1 & Т 30. & $\mathrm{~V} 1$ & 21 & -23 & 0 & 2 & 0.00 & 8 \\
\hline
\end{tabular}


Table 4. continued.

\begin{tabular}{|c|c|c|c|c|c|c|c|c|c|c|c|c|c|c|c|}
\hline $\begin{array}{c}\text { Dates } \\
\text { Phenomena } \\
\text { Locations } \\
\text { (1) }\end{array}$ & $\begin{array}{c}\text { Begins } \\
\mathrm{h} \mathrm{m} \mathrm{s} \\
\text { (2) }\end{array}$ & $\begin{array}{l}\text { Maxi. } \\
\mathrm{h} \mathrm{m} \text { s } \\
\text { (3) }\end{array}$ & $\begin{array}{c}\text { Ends } \\
\text { h m s } \\
\text { (4) }\end{array}$ & $\begin{array}{c}\text { Magn. } \\
\text { drop }\end{array}$ & $\begin{array}{l}\text { Ph. } \\
\text { (s) } \\
\text { (6) }\end{array}$ & $\begin{array}{l}\text { Dist. } \\
(\mathbf{R j})\end{array}$ & $\begin{array}{c}\text { C-o } \\
\text { (s) } \\
(8)\end{array}$ & $\begin{array}{c}\text { Ap. } \\
\text { (cm) }\end{array}$ & $\begin{array}{l}\text { Rec. } \\
\text { (10) }\end{array}$ & $\begin{array}{l}\text { El. } \\
\text { Jup. } \\
\left({ }^{\circ}\right) \\
(11)\end{array}$ & $\begin{array}{l}\text { El. } \\
\text { Sun } \\
\left({ }^{\circ}\right) \\
(12)\end{array}$ & (13) & $\begin{array}{l}\text { Filt. } \\
\text { (14) }\end{array}$ & $\begin{array}{c}\text { T. } \\
\text { int. } \\
\text { (s) } \\
\text { (15) }\end{array}$ & $\begin{array}{l}\text { Dia. } \\
(") \\
(16)\end{array}$ \\
\hline TORINO & & $\begin{array}{cc} & \pm 9 \\
1934 & 39 \\
+8\end{array}$ & & $\begin{array}{c}.081 \\
.055 \\
+.052\end{array}$ & & & 13 & Т 15. & CCD2 & 26 & -22 & 0 & 1 & 1. & - \\
\hline CATANIA & & $\begin{array}{rr}1934 & 37 \\
+7\end{array}$ & & $\begin{array}{l}.059 \\
+005 \\
+005\end{array}$ & & & 15 & Т 91. & PM & 34 & -30 & 0 & $2 \mathrm{~V}$ & 0.1 & 21. \\
\hline BUCHAREST1 & & $\begin{array}{cc}1934 & 38 \\
+14\end{array}$ & & $\begin{array}{l}.684 \\
.099\end{array}$ & & & 14 & L 38. & CCDI & 28 & -33 & 0 & $1 G G$ & 0.4 & - \\
\hline BOSKOOP & & 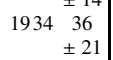 & & $\begin{array}{c} \pm .099 \\
.392 \\
\pm .082\end{array}$ & & & 16 & T 30. & CCDVX & 18 & -17 & 0 & - & 0.04 & - \\
\hline REUX & & 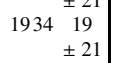 & & $\begin{array}{c}.086 \\
.586 \\
\pm .127\end{array}$ & & & 33 & т 30. & CCD5 & 20 & -18 & 0 & - & 0.04 & - \\
\hline LISBOA & & $\begin{array}{r}1934 \\
37 \\
\pm 39\end{array}$ & & $\begin{array}{c}.127 \\
.414 \\
\pm .163\end{array}$ & & & 15 & T 20. & CCD4 & 32 & -25 & 0 & - & 0.04 & - \\
\hline $\begin{array}{c}\text { OOSTERDUIN. } \\
\text { BORDEAUX }\end{array}$ & & $\begin{array}{cc}1934 & 43 \\
& \pm 26 \\
1934 & 35 \\
\pm 11\end{array}$ & & $\begin{array}{c}.045 \\
\pm .011 \\
.736 \\
\pm .076\end{array}$ & & & 17 & $\begin{array}{l}\text { T } 25 . \\
\text { T } 62 .\end{array}$ & $\begin{array}{l}\mathrm{CCD} 7 \\
\mathrm{CCD} 1\end{array}$ & $\begin{array}{l}19 \\
23\end{array}$ & $\begin{array}{l}-16 \\
-16\end{array}$ & 0 & $\begin{array}{c}2 \\
2 R\end{array}$ & 0.5 & - \\
\hline $\begin{array}{l}97 / 9 / 19 \\
403(P) \\
\end{array}$ & $\begin{array}{lll}22 & 8 & 16 \\
\end{array}$ & $2214 \quad 47$ & 222120 & .061 & -13.3 & 14.8 & & & & & & & & & \\
\hline $\begin{array}{c}\text { UCCLE } \\
\text { BORDEAUX }\end{array}$ & & $\begin{array}{cc}2218 & 23 \\
& \pm 177 \\
2215 & 13 \\
& \pm 84 \\
\end{array}$ & & $\begin{array}{c}.00 .095 \\
\pm .063 \\
.092 \\
\pm .049 \\
\end{array}$ & & & -26 & $\begin{array}{l}\text { T } 85 . \\
\text { T } 62 .\end{array}$ & $\begin{array}{l}\text { CCD8 } \\
\text { CCD1 }\end{array}$ & 25 & -35 & 0 & $\begin{array}{c}2 \\
3 R\end{array}$ & $\begin{array}{c}0.06 \\
0.5\end{array}$ & - \\
\hline $\begin{array}{l}97 / 9 / 21 \\
3 \mathrm{E1}(\mathrm{P}) \\
\end{array}$ & 202745 & $2031 \quad 11$ & 203438 & .726 & 3.8 & 3.0 & & & & & & & & & \\
\hline MEUDON & & $\begin{array}{cc}2031 & 1 \\
& \pm 2\end{array}$ & & $\begin{array}{l}1.533 \\
\pm .046\end{array}$ & & & 10 & Т 100. & V3 & 23 & -26 & 0 & 1 & 0.04 & - \\
\hline PRAHA & & $\begin{array}{cc}2031 & 4 \\
\pm 6\end{array}$ & & $\begin{array}{l}1.259 \\
\pm .137\end{array}$ & & & 7 & L 19. & $\mathrm{v} 1$ & 21 & -31 & 0 & 2 & 0.00 & 8 \\
\hline STUTTGART & & $\begin{array}{cc}2034 & 3 \\
\pm 2\end{array}$ & & $\begin{array}{l}1.337 \\
\pm .045\end{array}$ & & & 46 & Т 30. & CCD11 & 23 & -29 & 0 & $2 R$ & 1. & - \\
\hline CACERES & & $\begin{array}{cc}2031 & 0 \\
\pm 1\end{array}$ & & $\begin{array}{l}1.564 \\
\pm .028\end{array}$ & & & 11 & $\mathrm{~T} 25$. & CCD & 31 & -25 & 0 & V & $2-7$ & - \\
\hline CHATEAUGIRON & & $\begin{array}{cc}2031 & 2 \\
\pm 3\end{array}$ & & $\begin{array}{c}.518 \\
\pm .060\end{array}$ & & & 9 & T 21. & CCD10 & 24 & -24 & 0 & 2 & 1.2 & - \\
\hline PIC-DU-MIDI & & $\begin{array}{cc}2031 & 1 \\
& \pm 2\end{array}$ & & $\begin{array}{c}.397 \\
\pm .011\end{array}$ & & & 10 & Т 100. & CCDI & 29 & -28 & 0 & $2 \mathrm{~V}$ & 0.1 & - \\
\hline ZARAGOZA & & $\begin{array}{cc}2031 & 2 \\
\pm 3\end{array}$ & & $\begin{array}{l}1.263 \\
\pm .050\end{array}$ & & & 9 & Т 15. & $\mathrm{CCD}$ & 29 & -23 & 0 & 2 & - & - \\
\hline OCA & & $\begin{array}{cc}2031 & 7 \\
\pm 9\end{array}$ & & $\begin{array}{c}.069 \\
.969 \\
\pm .111\end{array}$ & & & 4 & Т 150. & PM7 & 28 & -31 & 0 & $B$ & 0.2 & - \\
\hline $\mathrm{OCA}$ & & 203052 & & $\begin{array}{l}1.168 \\
+068\end{array}$ & & & 19 & Т 150. & PM7 & 28 & -31 & 0 & $R$ & 0.2 & - \\
\hline OCA & & $\begin{array}{cc} \pm 3 \\
2030 \\
50\end{array}$ & & $\begin{array}{l} \pm .008 \\
1.288 \\
+149\end{array}$ & & & 21 & Т 150. & PM7 & 28 & -31 & 0 & v & 0.2 & - \\
\hline UCCLE & & $\begin{array}{r}2030 \quad 59 \\
\pm 7\end{array}$ & & $\begin{array}{l} \pm .149 \\
1.739 \\
\pm .0191\end{array}$ & & & 6 & $\mathrm{~T} 85$. & CCD8 & 21 & -26 & 0 & 2 & 0.06 & - \\
\hline BUCHAREST1 & & $\begin{array}{cc}2031 & 0 \\
\pm 3\end{array}$ & & $\begin{array}{r}1.538 \\
\pm .082\end{array}$ & & & 11 & L 38. & CCDI & 25 & -40 & 0 & $1 G G$ & 0.4 & - \\
\hline BUCHAREST2 & & $\begin{array}{cc}2030 & 56 \\
\pm 4\end{array}$ & & $\begin{array}{r}1.540 \\
\pm .127\end{array}$ & & & 15 & L 38. & CCDST & 25 & -40 & 0 & $2 \mathrm{~V}$ & 3. & - \\
\hline BOSKOOP & & $\begin{array}{cc}2031 & 5 \\
\pm 10\end{array}$ & & $\begin{array}{l}1.100 \\
\pm .189\end{array}$ & & & 6 & Т 30. & CCDVX & 20 & -25 & 0 & - & 0.04 & \\
\hline REUX & & 2031 3 & & $\begin{array}{l}3.789 \\
\pm .771\end{array}$ & & & 8 & Т 30. & CCD5 & 22 & -26 & 0 & - & 0.04 & - \\
\hline WILP-ACHTER. & & $\begin{array}{cc}2031 & 0 \\
& \pm 16\end{array}$ & & $\begin{array}{l}4.224 \\
\pm .935\end{array}$ & & & 11 & Т 20. & V2 & 20 & -26 & 0 & 3 & 0.04 & - \\
\hline OOSTERDUIN. & & $\begin{array}{c}2031 \\
\quad 6 \\
\pm 16\end{array}$ & & $\begin{array}{r}1.529 \\
\pm .312\end{array}$ & & & 5 & T 25. & $\mathrm{CCD} 7$ & 21 & -25 & 0 & 2 & 0.04 & - \\
\hline LISBOA & & $2031 \quad 00$ & & $\begin{array}{l}1.612 \\
+046\end{array}$ & & & 11 & T 25. & $\mathrm{CCD} 2$ & 31 & -23 & 0 & - & - & - \\
\hline MUNICH & & $\begin{array}{r}2031 \\
5 \\
\pm 9 \\
\end{array}$ & & $\begin{array}{r} \pm .040 \\
1.568 \\
\pm .239 \\
\end{array}$ & & & 6 & т 28. & CCDM & 23 & -31 & 0 & $1 * 4$ & - & - \\
\hline $\begin{array}{l}97 / 9 / 22 \\
3 \mathrm{E} 2(\mathrm{P}) \\
\end{array}$ & 185422 & $191 \quad 56$ & 19924 & .673 & 8.6 & 9.0 & & & & & & & & & \\
\hline OOSTERDUIN. & & $\begin{array}{ll}192 & 3 \\
\pm 110\end{array}$ & & $\begin{array}{r}.367 \\
\pm .267\end{array}$ & & & -7 & T 25. & CCD7 & 17 & -13 & 0 & 3 & 0.04 & - \\
\hline MEUDON & & $\begin{array}{ccc}19 & 2 & 3 \\
& \pm 10\end{array}$ & & $\begin{array}{l}1.040 \\
\pm .059\end{array}$ & & & -7 & Т 100. & CCDVX & 19 & -13 & 0 & 1 & 0.04 & - \\
\hline PRAHA & & 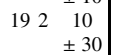 & & $\begin{array}{c}.837 \\
\pm .154\end{array}$ & & & -14 & L 19. & $\mathrm{~V} 1$ & 21 & -20 & 0 & 2 & 0.00 & 8 \\
\hline CHATEAUGIRON & & 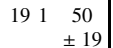 & & $\begin{array}{l}1.001 \\
\pm .117\end{array}$ & & & 6 & T 21. & CCD10 & 19 & -11 & 0 & 2 & 0.6 & - \\
\hline ZARAGOZA & & $\left.\begin{array}{ccc}19 & 2 & 3 \\
+3\end{array}\right]$ & & $\begin{array}{r}1.062 \\
+.022\end{array}$ & & & -7 & $\mathrm{~T} 15$. & $\mathrm{CCD}$ & 22 & -7 & 0 & 2 & - & - \\
\hline UCCLE & & $\begin{array}{ccc}19 & 2 & 7 \\
+22\end{array}$ & & $\begin{array}{l}1.402 \\
+020\end{array}$ & & & -8 & $\mathrm{~T} 85$. & CCD8 & 18 & -14 & 0 & $2 * 1$ & 0.06 & - \\
\hline BUCHAREST1 & & 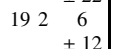 & & $\begin{array}{r}1.087 \\
+072\end{array}$ & & & -10 & L 38. & CCDI & 28 & -30 & 0 & $1 G G$ & 0.4 & - \\
\hline BUCHAREST2 & & $\begin{array}{ll}19 & 2 \\
& 19\end{array}$ & & $\begin{array}{l}1.013 \\
+069\end{array}$ & & & -23 & L 38. & CCDST & 28 & -30 & 0 & $1 \mathrm{~V}$ & 2. & - \\
\hline BOSKOOP & & $\begin{array}{ccc}19 & 111 \\
19 & 59 \\
& \pm 24\end{array}$ & & $\begin{array}{c} \pm .069 \\
.919 \\
\pm .140\end{array}$ & & & -3 & T 30. & CCDVX & 17 & -13 & 0 & - & 0.04 & - \\
\hline REUX & & $\begin{array}{ccc}19 & 1 & 56 \\
& \pm 29\end{array} \mid$ & & $\begin{array}{l} \pm .140 \\
1.171 \\
\pm .217\end{array}$ & & & 0 & T 30. & CCD5 & 19 & -14 & 0 & - & 0.04 & - \\
\hline
\end{tabular}


J.-E. Arlot et al.: The PHEMU97 Campaign, Online Material p 10

Table 4. continued.

\begin{tabular}{|c|c|c|c|c|c|c|c|c|c|c|c|c|c|c|c|}
\hline $\begin{array}{c}\text { Dates } \\
\text { Phenomena } \\
\text { Locations } \\
\text { (1) }\end{array}$ & $\begin{array}{l}\text { Begins } \\
\mathrm{h} \mathrm{m} \mathrm{s} \\
(2)\end{array}$ & $\begin{array}{c}\text { Maxi. } \\
\text { h m s } \\
\text { (3) }\end{array}$ & $\begin{array}{l}\text { Ends } \\
\text { h m s } \\
\text { (4) }\end{array}$ & $\begin{array}{c}\text { Magn. } \\
\text { drop } \\
(5)\end{array}$ & $\begin{array}{l}\text { Ph. } \\
(s) \\
(6)\end{array}$ & $\begin{array}{l}\text { Dist. } \\
(\mathbf{R j}) \\
(7)\end{array}$ & $\begin{array}{l}\text { C-O } \\
(\mathrm{s}) \\
(8)\end{array}$ & $\begin{array}{l}\text { Ap. } \\
(\mathbf{c m}) \\
(9)\end{array}$ & (10) & $\begin{array}{l}\text { El. } \\
\text { Jup. } \\
\left({ }^{\circ}\right) \\
(11)\end{array}$ & $\begin{array}{l}\text { El. } \\
\text { Sun } \\
\left({ }^{\circ}\right) \\
(12)\end{array}$ & (13) & $\begin{array}{l}\text { Filt. } \\
\text { (14) }\end{array}$ & $\begin{array}{l}\text { T. } \\
\text { int. } \\
\text { (s) } \\
(15)\end{array}$ & $\begin{array}{l}\text { Dia. } \\
\text { (") } \\
\text { (16) }\end{array}$ \\
\hline WILP-ACHTER. & & $\begin{array}{cc}191 & 56 \\
& \pm 59\end{array}$ & & $\begin{array}{l}2.956 \\
\pm .961\end{array}$ & & & 0 & T 20. & V2 & 17 & -14 & 0 & 3 & 0.04 & - \\
\hline BORDEAUX & & $\begin{array}{cc}192 & 2 \\
& \pm 7\end{array}$ & & $\begin{array}{r}1.082 \\
+.038\end{array}$ & & & -6 & T 62. & CCD1 & 22 & -12 & 0 & $2 R$ & 1.5 & - \\
\hline MUNICH & & 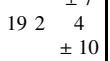 & & $\begin{array}{r}1.049 \\
\pm .062\end{array}$ & & & -8 & Т 28. & $\mathrm{CCDM}$ & 22 & -19 & 0 & $1 * 4$ & - & - \\
\hline KAVALUR & & $\begin{array}{cc}192 & 7 \\
& \pm 10 \\
\end{array}$ & & $\begin{array}{r}1.422 \\
\pm .109 \\
\end{array}$ & & & -11 & Т 102. & $\mathrm{CCDC}$ & 31 & -76 & 0 & $1 * 3$ & - & - \\
\hline $\begin{array}{l}97 / 9 / 23 \\
102(\mathrm{P})\end{array}$ & 2266 & $227 \quad 10$ & $\begin{array}{llll}22 & 8 & 14\end{array}$ & .002 & -2.0 & 5.9 & & & & & & & & & \\
\hline CATANIA & & $\begin{array}{cc}228 & 39 \\
& \pm 282 \\
\end{array}$ & & $\begin{array}{c}.001 \\
\pm .006 \\
\end{array}$ & & & -89 & Т 91. & PM & 25 & -51 & 0 & $2 \mathrm{~V}$ & 0.1 & 21. \\
\hline $\begin{array}{l}97 / 9 / 25 \\
103(P) \\
\end{array}$ & 195150 & $1954 \quad 25$ & 195658 & .063 & -5.7 & 5.2 & & & & & & & & & \\
\hline LUMEZZANE & & $\begin{array}{cc}1955 & 45 \\
& \pm 8\end{array}$ & & $\begin{array}{c}.130 \\
\pm .036\end{array}$ & & & -80 & T 40. & PM & 26 & -28 & 0 & 2 & 0.1 & 129. \\
\hline OHP & & $\begin{array}{rc}1953 & 56 \\
& \pm 60\end{array}$ & & $\begin{array}{l}.167 \\
\pm .047\end{array}$ & & & 29 & T 80. & CCD1 & 28 & -26 & 0 & 2 & 1.5 & - \\
\hline MEUDON & & & & $\begin{array}{c}.082 \\
\pm .048\end{array}$ & & & -7 & Т 100. & v3 & 23 & -22 & 0 & 1 & 0.04 & - \\
\hline PARIS & & $\begin{array}{r}1954 \\
\pm 100\end{array}$ & & $\begin{array}{c}.061 \\
\pm .096\end{array}$ & & & 4 & L 38. & & 23 & -22 & 0 & $3 \mathrm{~V}$ & 0.3 & - \\
\hline UCCLE & & $\begin{array}{r}1954 \quad 34 \\
\pm 136\end{array}$ & & $\begin{array}{l}0.070 \\
\pm .162\end{array}$ & & & -22 & T 85. & CCD8 & 21 & -22 & 0 & $3 * 1$ & 0.06 & - \\
\hline BOSKOOP & & $\begin{array}{rc}1954 & 28 \\
\pm & 145\end{array}$ & & $\begin{array}{c}.075 \\
\pm .101\end{array}$ & & & -3 & Т 30. & CCDVX & 20 & -22 & 0 & - & 0.04 & - \\
\hline MUNICH & & $\begin{array}{rc}1954 & 44 \\
& \pm 72 \\
\end{array}$ & & $\begin{array}{c}.061 \\
\pm .070 \\
\end{array}$ & & & -19 & Т 28. & $\mathrm{CCDM}$ & 24 & -28 & 0 & $3 * 4$ & - & - \\
\hline $\begin{array}{r}97 / 9 / 25 \\
1 \mathrm{E3}(\mathrm{A}) \\
\end{array}$ & 222821 & $2232 \quad 35$ & 223648 & .449 & 7.1 & 6.5 & & & & & & & & & \\
\hline MUNICH & & $\begin{array}{rc}2232 & 22 \\
& \pm 15\end{array}$ & & $\begin{array}{c}.614 \\
\pm .116\end{array}$ & & & 13 & T 28. & $\mathrm{CCDM}$ & 15 & -42 & 0 & $1 * 4$ & - & - \\
\hline BOSKOOP & & $\begin{array}{rc}2232 & 17 \\
& \pm 23\end{array}$ & & $\begin{array}{c}.492 \\
\pm .147\end{array}$ & & & 18 & Т 30. & CCDVX & 14 & -38 & 0 & - & 0.04 & - \\
\hline REUX & & $\begin{array}{rr}2232 & 15 \\
\pm 27\end{array}$ & & $\begin{array}{c}.541 \\
\pm .197\end{array}$ & & & 20 & T 30. & CCD5 & 16 & -39 & 0 & - & 0.04 & - \\
\hline WILP-ACHTER. & & $\begin{array}{rc}2232 & 13 \\
\pm 67\end{array}$ & & $\begin{array}{c}.603 \\
\pm .433\end{array}$ & & & 22 & Т 20. & $\mathrm{~V} 2$ & 14 & -38 & 0 & - & 0.04 & - \\
\hline BORDEAUX & & & & $\begin{array}{c}.668 \\
\pm .057\end{array}$ & & & 10 & T 62. & CCD1 & 22 & -43 & 0 & $2 R$ & 0.5 & - \\
\hline PRAHA & & $\begin{array}{rc}2232 & 27 \\
\pm & 28\end{array}$ & & $\begin{array}{c}.650 \\
\pm .184\end{array}$ & & & 8 & L 19. & $\mathrm{~V} 1$ & 12 & -41 & 0 & 2 & 0.00 & 8 \\
\hline STUTTGART & & $\begin{array}{rc}2232 & 14 \\
& \pm 9\end{array}$ & & $\begin{array}{c}.666 \\
\pm .076\end{array}$ & & & 21 & Т 30. & CCD11 & 15 & -42 & 0 & $2 R$ & 2. & - \\
\hline LUMEZZANE & & $\begin{array}{rc}2233 & 26 \\
& \pm 36\end{array}$ & & $\begin{array}{l}.161 \\
\pm .055\end{array}$ & & & -51 & T 40. & PM & 17 & -45 & 0 & 2 & 0.1 & 129. \\
\hline OHP & & $\begin{array}{cc}2232 & 17 \\
& \pm 8\end{array}$ & & $\begin{array}{c}.651 \\
\pm .053\end{array}$ & & & 18 & T 80. & $\mathrm{CCD} 1$ & 21 & -46 & 0 & 2 & 1.5 & - \\
\hline PARIS & & $\begin{array}{rc}2232 & 17 \\
& \pm 11\end{array}$ & & $\begin{array}{c}.609 \\
\pm .069\end{array}$ & & & 18 & L 38 . & & 18 & -40 & 0 & $1 \mathrm{~V}$ & 0.3 & - \\
\hline PIC-DU-MIDI & & $\begin{array}{cc}2232 & 31 \\
& \pm 5\end{array}$ & & $\begin{array}{c}.641 \\
\pm .026\end{array}$ & & & 4 & Т 100. & CCDI & 24 & -45 & 0 & $2 K$ & 0.1 & - \\
\hline OCA & & $\begin{array}{ll}2232 & 11 \\
& \pm 8\end{array}$ & & $\begin{array}{c}.659 \\
\pm .056\end{array}$ & & & 24 & Т 150. & PM7 & 20 & -46 & 0 & $B$ & 0.2 & - \\
\hline OCA & & & & $\begin{array}{c}.667 \\
\pm .126\end{array}$ & & & 12 & Т 150. & PM7 & 20 & -46 & 0 & $R$ & 0.2 & - \\
\hline OCA & & & & $\begin{array}{c}.674 \\
\pm .118\end{array}$ & & & 28 & Т 150. & PM7 & 20 & -46 & 0 & $V$ & 0.2 & - \\
\hline UCCLE & & & & & & & 12 & T 85. & CCD8 & 15 & -39 & 0 & $3 * 1$ & 0.06 & - \\
\hline ESSEN & & $\begin{array}{rc}2232 & 16 \\
\pm 11 \\
\end{array}$ & & $\begin{array}{c}.669 \\
\pm .095 \\
\end{array}$ & & & 19 & Т 36. & PM6 & 14 & -38 & 0 & $1 \mathrm{~V}$ & 0.2 & 105. \\
\hline $\begin{array}{l}97 / 9 / 28 \\
3 \text { E1(P) } \\
\end{array}$ & 23144 & $2317 \quad 26$ & 232047 & .483 & 4.3 & 3.7 & & & & & & & & & \\
\hline PIC-DU-MIDI & & $\begin{array}{rc}2317 & 18 \\
& \pm 5\end{array}$ & & $\begin{array}{c}.753 \\
\pm .055\end{array}$ & & & 8 & T 100. & CCDI & 18 & -49 & 0 & $3 K$ & 0.1 & - \\
\hline CATANIA & & $\begin{array}{rc}2318 & 1 \\
& \pm 14 \\
\end{array}$ & & $\begin{array}{c}.063 \\
\pm .011 \\
\end{array}$ & & & -35 & Т 91. & PM & 12 & -54 & 0 & $1 \mathrm{~V}$ & 0.1 & 21. \\
\hline $\begin{array}{l}97 / 9 / 29 \\
302(P) \\
\end{array}$ & 183538 & $1842 \quad 19$ & 184856 & .196 & -9.8 & 9.3 & & & & & & & & & \\
\hline CHATEAUGIRON & & $\begin{array}{rc}1842 & 28 \\
\pm 45\end{array}$ & & $\begin{array}{c}.394 \\
\pm .013\end{array}$ & & & -9 & T 21. & CCD10 & 20 & -10 & 0 & 3 & 0.6 & - \\
\hline MEUDON & & $\begin{array}{cc}1840 & 49 \\
& \pm 27\end{array}$ & & $\begin{array}{c}.973 \\
\pm .129\end{array}$ & & & 90 & Т 100. & V3 & 20 & -12 & 0 & 2 & 0.04 & - \\
\hline PIC-DU-MIDI & & $\begin{array}{r}1842 \\
52 \\
\pm 33\end{array}$ & & $\begin{array}{c}.173 \\
\pm .033\end{array}$ & & & -33 & Т 100. & CCDI & 25 & -12 & 0 & $2 K$ & 0.1 & - \\
\hline CATANIA & & $\begin{array}{r}1842 \\
\quad 39 \\
\pm 19\end{array}$ & & $\begin{array}{c}.070 \\
\pm .006\end{array}$ & & & -20 & Т 91. & PM & 33 & -24 & 0 & $1 \mathrm{~V}$ & 0.1 & 21. \\
\hline UCCLE & & $\begin{array}{rc}1842 & 42 \\
\pm 55\end{array}$ & & $\begin{array}{c}.355 \\
\pm .103\end{array}$ & & & -24 & T 85. & CCD8 & 19 & -13 & 0 & 2 & 0.06 & - \\
\hline BUCHAREST1 & & $\begin{array}{rc}1842 & 45 \\
& \pm 39\end{array}$ & & $\begin{array}{c}.345 \\
\pm .059\end{array}$ & & & -26 & L 38. & CCDI & 28 & -29 & 0 & $1 G G$ & 0.4 & - \\
\hline
\end{tabular}


Table 4. continued.

\begin{tabular}{|c|c|c|c|c|c|c|c|c|c|c|c|c|c|c|c|}
\hline $\begin{array}{c}\text { Dates } \\
\text { Phenomena } \\
\text { Locations } \\
\text { (1) }\end{array}$ & $\begin{array}{c}\begin{array}{c}\text { Begins } \\
\mathrm{h} \mathrm{m} \mathrm{s} \\
\text { (2) }\end{array} \\
\end{array}$ & $\begin{array}{c}\text { Maxi. } \\
\text { h m s } \\
\text { (3) }\end{array}$ & $\begin{array}{c}\text { Ends } \\
\text { h m s } \\
\text { (4) }\end{array}$ & $\begin{array}{c}\text { Magn. } \\
\text { drop }\end{array}$ & $\begin{array}{l}\text { Ph. } \\
\text { (s) } \\
\text { (6) }\end{array}$ & $\begin{array}{l}\text { Dist. } \\
(\mathbf{R j})\end{array}$ & $\begin{array}{l}\text { C-O } \\
\text { (s) } \\
(8) \\
\end{array}$ & 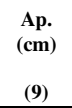 & $\begin{array}{l}\text { Rec. } \\
\text { (10) }\end{array}$ & $\begin{array}{c}\text { El. } \\
\text { Jup. } \\
\left({ }^{\circ}\right) \\
(11)\end{array}$ & $\begin{array}{l}\text { El. } \\
\text { Sun } \\
\left({ }^{\prime}\right) \\
(12)\end{array}$ & $\begin{array}{l}\text { Cd. } \\
\text { (13) }\end{array}$ & (14) & $\begin{array}{c}\mathrm{T} . \\
\text { int. } \\
(\mathbf{s}) \\
(15)\end{array}$ & $\begin{array}{l}\text { Dia. } \\
(") \\
(16)\end{array}$ \\
\hline $\begin{array}{c}\text { BUCHAREST2 } \\
\text { OHP }\end{array}$ & & $\begin{array}{cc}1842 & 18 \\
& \pm 21 \\
1843 & 2 \\
& 2 \\
& \pm 24 \\
\end{array}$ & & $\begin{array}{c}.409 \\
\pm .050 \\
.476 \\
\pm .041 \\
\end{array}$ & & & -43 & $\begin{array}{l}\mathrm{L} 38 . \\
\mathrm{T} 80 .\end{array}$ & $\begin{array}{l}\text { CCDST } \\
\text { CCD1 }\end{array}$ & $\begin{array}{l}28 \\
25\end{array}$ & $\begin{array}{l}-29 \\
-15\end{array}$ & $\begin{array}{l}0 \\
0\end{array}$ & $\begin{array}{l}1 V \\
3 V\end{array}$ & $\begin{array}{l}2 . \\
5 .\end{array}$ & - \\
\hline $\begin{array}{l}97 / 9 / 29 \\
3 \mathrm{E} 2(\mathrm{P}) \\
\end{array}$ & 224042 & $2246 \quad 24$ & 22527 & .229 & 7.1 & 8.7 & & & & & & & & & \\
\hline $\begin{array}{c}\text { PIC-DU-MIDI } \\
\text { BORDEAUX } \\
\text { MUNICH }\end{array}$ & & $\begin{array}{cc}2246 & 32 \\
& \pm 29 \\
2246 & 33 \\
& \pm 31 \\
2246 & 34 \\
& \pm 60 \\
\end{array}$ & & $\begin{array}{c}.173 \\
\pm .029 \\
.226 \\
\pm .047 \\
.235 \\
\pm .119 \\
\end{array}$ & & & $\begin{array}{c}-8 \\
-9 \\
-10\end{array}$ & $\begin{array}{l}\text { T } 100 . \\
\text { T } 62 . \\
\text { T } 28 .\end{array}$ & $\begin{array}{l}\text { CCDI } \\
\text { CCD1 } \\
\text { CCDM }\end{array}$ & 12 & $\begin{array}{l}-47 \\
-46 \\
-44\end{array}$ & 0 & $\begin{array}{l}2 K \\
2 R \\
2 * 4\end{array}$ & 0.1 & - \\
\hline $\begin{array}{l}97 / 10 / 2 \\
103(P) \\
\end{array}$ & 223813 & $2240 \quad 14$ & 224216 & . 041 & -5.0 & 4.7 & & & & & & & & & \\
\hline $\begin{array}{l}\text { CATANIA } \\
\text { BORDEAUX }\end{array}$ & & $\begin{array}{cc}2241 & 15 \\
& \pm 79 \\
2240 & 35 \\
& \pm 55 \\
\end{array}$ & & $\begin{array}{c}.005 \\
\pm .011 \\
.181 \\
\pm .086 \\
\end{array}$ & & & $\begin{array}{l}-61 \\
-21\end{array}$ & $\begin{array}{l}\text { T } 91 . \\
\text { T } 62 .\end{array}$ & $\begin{array}{c}\text { PM } \\
\text { CCD1 }\end{array}$ & 19 & $\begin{array}{l}-56 \\
-46\end{array}$ & 0 & $\begin{array}{l}1 V \\
3 R\end{array}$ & $\begin{array}{c}0.1 \\
1 .\end{array}$ & 22. \\
\hline $\begin{array}{l}97 / 10 / 3 \\
1 \mathrm{E3}(\mathrm{A}) \\
\end{array}$ & 12136 & $\begin{array}{l}125 \quad 21 \\
\end{array}$ & 1299 & .335 & & & & & & & & & & & \\
\hline $\begin{array}{c}\text { NEW-YORK } \\
\text { BOWIE }\end{array}$ & & $\begin{array}{cc}125 & 2 \\
& \pm 9 \\
125 & 3 \\
& \pm 4 \\
\end{array}$ & & $\begin{array}{c}.448 \\
\pm .056 \\
.444 \\
\pm .023 \\
\end{array}$ & & & 19 & $\begin{array}{l}\text { Т } 20 . \\
\text { Т } 20 .\end{array}$ & $\begin{array}{l}\mathrm{CCD} 2 \\
\mathrm{CCD} 2\end{array}$ & $\begin{array}{l}29 \\
33\end{array}$ & $\begin{array}{l}-29 \\
-31\end{array}$ & $\begin{array}{l}0 \\
0\end{array}$ & $\begin{array}{l}2 V \\
2 V\end{array}$ & $\begin{array}{l}1 . \\
4 .\end{array}$ & $\begin{array}{l}10 . \\
14 .\end{array}$ \\
\hline $\begin{array}{l}97 / 10 / 5 \\
301(\mathrm{P}) \\
\end{array}$ & 232348 & $2326 \quad 4$ & 232820 & .141 & -4.6 & 2.4 & & & & & & & & & \\
\hline ESO & & $\begin{array}{cc}2326 & 2 \\
& \pm 3 \\
\end{array}$ & & $\begin{array}{r}.356 \\
\pm .017 \\
\end{array}$ & & & 2 & Т 220. & $\mathrm{CCD} 3$ & 69 & -10 & 0 & $1 * 2$ & 0.5 & - \\
\hline $\begin{array}{r}97 / 10 / 6 \\
302(\mathrm{P}) \\
\end{array}$ & $\begin{array}{lll}22 & 622 \\
\end{array}$ & $2212 \quad 30$ & 221836 & .217 & -9.5 & 9.3 & & & & & & & & & \\
\hline $\begin{array}{l}\text { TENERIFE } \\
\text { CATANIA }\end{array}$ & & $\begin{array}{cc}2212 & 41 \\
& \pm 17 \\
2213 & 9 \\
& \pm 45 \\
\end{array}$ & & $\begin{array}{c}.358 \\
\pm .035 \\
.031 \\
\pm .007 \\
\end{array}$ & & & $\begin{array}{l}-11 \\
-39\end{array}$ & $\begin{array}{l}\text { T } 80 . \\
\text { Т } 91 .\end{array}$ & $\begin{array}{c}\text { CCDW } \\
\text { PM }\end{array}$ & 41 & $\begin{array}{l}-46 \\
-57\end{array}$ & $\begin{array}{l}0 \\
0\end{array}$ & $\begin{array}{c}B \\
2 V\end{array}$ & $\begin{array}{c}1 . \\
0.1\end{array}$ & 42. \\
\hline $\begin{array}{l}97 / 10 / 7 \\
401(\mathrm{P}) \\
\end{array}$ & 19346 & $1935 \quad 43$ & 193720 & . 028 & -3.9 & 3.7 & & & & & & & & & \\
\hline $\begin{array}{c}\text { PARIS } \\
\text { NAUCHNY }\end{array}$ & & $\begin{array}{cc}1937 & 6 \\
& \pm 28 \\
1935 \quad 18 \\
\pm 35 \\
\end{array}$ & & $\begin{array}{c}.211 \\
\pm .102 \\
.464 \\
\pm .338 \\
\end{array}$ & & & -83 & $\begin{array}{l}\text { L } 38 . \\
\text { T } 125 .\end{array}$ & $\begin{array}{l}\text { PM2 } \\
\text { PM5 }\end{array}$ & 23 & $\begin{array}{l}-23 \\
-44\end{array}$ & 0 & $\begin{array}{l}3 V \\
3 V\end{array}$ & 0.3 & 28. \\
\hline $\begin{array}{r}97 / 10 / 16 \\
203(P) \\
\end{array}$ & 173346 & $1736 \quad 23$ & 17391 & .208 & -7.6 & 2.0 & & & & & & & & & \\
\hline TORINO & & $\begin{array}{r}1736 \quad 33 \\
\quad \pm 15 \\
\end{array}$ & & $\begin{array}{r}.232 \\
\pm .032 \\
\end{array}$ & & & -10 & Т 15. & CCD2 & 25 & -11 & 0 & 1 & 1.5 & - \\
\hline $\begin{array}{l}97 / 10 / 18 \\
102(\mathrm{P}) \\
\end{array}$ & 182040 & $1822 \quad 22$ & 18244 & .024 & -4.1 & 5.7 & & & & & & & & & \\
\hline UCCLE & & $\begin{array}{rr}1823 & 12 \\
& \pm 599 \\
\end{array}$ & & $\begin{array}{c}.012 \\
\pm .091 \\
\end{array}$ & & & 75 & Т 85. & CCD8 & 21 & -16 & 0 & $2 * 1$ & 0.06 & - \\
\hline $\begin{array}{l}97 / 10 / 20 \\
301(P) \\
\end{array}$ & $\begin{array}{l}44324 \\
\end{array}$ & $445 \quad 53$ & 44821 & .138 & -5.6 & 3.6 & & & & & & & & & \\
\hline TOPEKA & & $\begin{array}{rr}447 \quad 2 \\
\quad+58 \\
\end{array}$ & & $\begin{array}{c}.004 \\
\pm .004 \\
\end{array}$ & & & 69 & L 38 . & CCD12 & 17 & -56 & 0 & 2 & - & - \\
\hline $\begin{array}{l}97 / 10 / 23 \\
203(\mathbf{P}) \\
\end{array}$ & 204018 & $2042 \quad 58$ & 204539 & .219 & -8.0 & 2.4 & & & & & & & & & \\
\hline TENERIFE & & $\begin{array}{r}2043 \quad 33 \\
\quad \pm 28 \\
\end{array}$ & & $\begin{array}{c}.083 \\
\pm .027 \\
\end{array}$ & & & -35 & T 80. & CCDW & 42 & -30 & 0 & $B$ & 1. & - \\
\hline $\begin{array}{l}97 / 10 / 24 \\
403(P) \\
\end{array}$ & 95832 & $103 \quad 7$ & 10741 & .445 & -10.7 & 4.8 & & & & & & & & & \\
\hline FUNAHO & & $\begin{array}{rr}103 & 19 \\
& \pm 4 \\
\end{array}$ & & $\begin{array}{c}.707 \\
\pm .033 \\
\end{array}$ & & & -12 & T 28. & PM3 & 37 & -22 & 0 & $2 \mathrm{~V}$ & 0.2 & - \\
\hline $\begin{array}{l}97 / 11 / 3 \\
301(\mathbf{P}) \\
\end{array}$ & 102420 & $1027 \quad 29$ & 103040 & . 190 & -7.3 & 4.8 & & & & & & & & & \\
\hline $\begin{array}{l}\text { FUNAHO } \\
\text { OKAYAMA }\end{array}$ & & $\begin{array}{rr}1027 & 37 \\
& \pm 9 \\
1027 & 38 \\
& \pm 5 \\
\end{array}$ & & $\begin{array}{c}.272 \\
\pm .023 \\
.274 \\
\pm .013\end{array}$ & & & $\begin{array}{l}-8 \\
-9\end{array}$ & $\begin{array}{l}\text { T } 28 . \\
\text { Т } 35 .\end{array}$ & $\begin{array}{l}\text { PM3 } \\
\text { PM3 }\end{array}$ & 35 & $\begin{array}{l}-29 \\
-29\end{array}$ & 0 & $2 \mathrm{~V}$ & 0.2 & 40. \\
\hline
\end{tabular}


J.-E. Arlot et al.: The PHEMU97 Campaign, Online Material p 12

Table 4. continued.

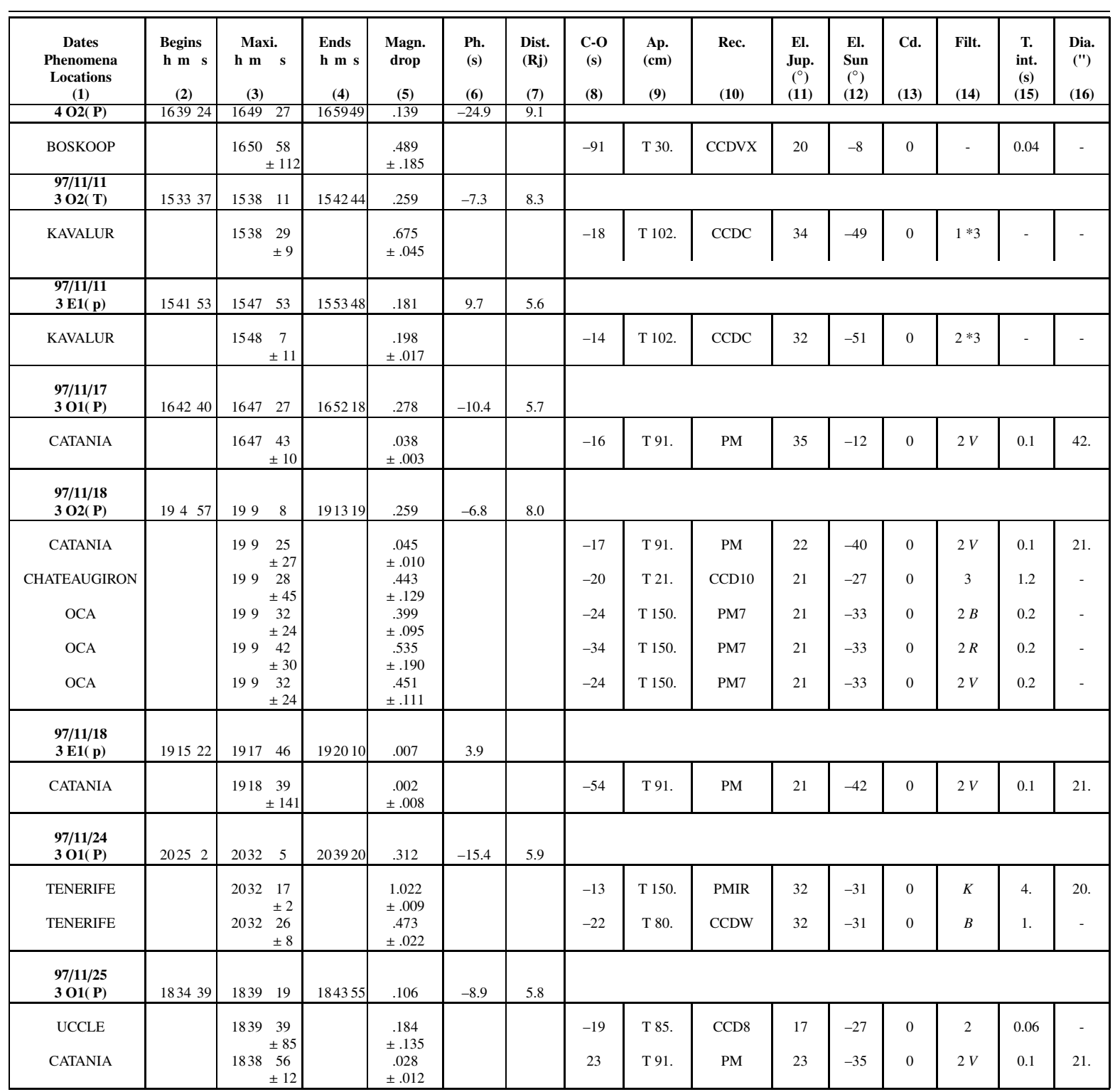


J.-E. Arlot et al.: The PHEMU97 Campaign, Online Material p 13
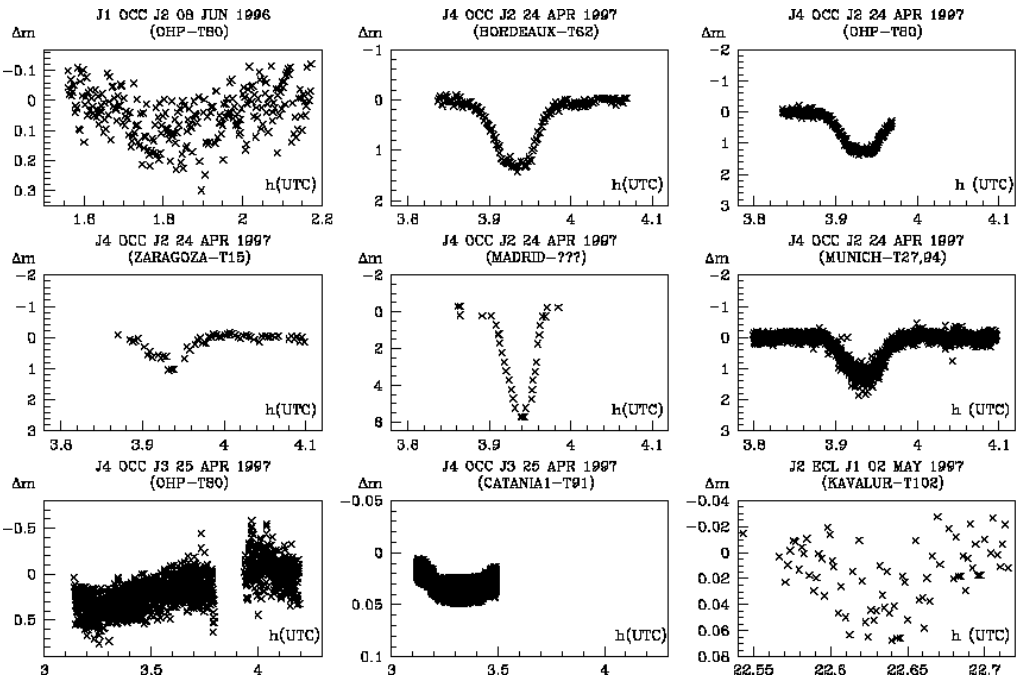

Fig. 1. Lightcurves from the observations of the mutual events of the Galilean satellites in 1996-1997.
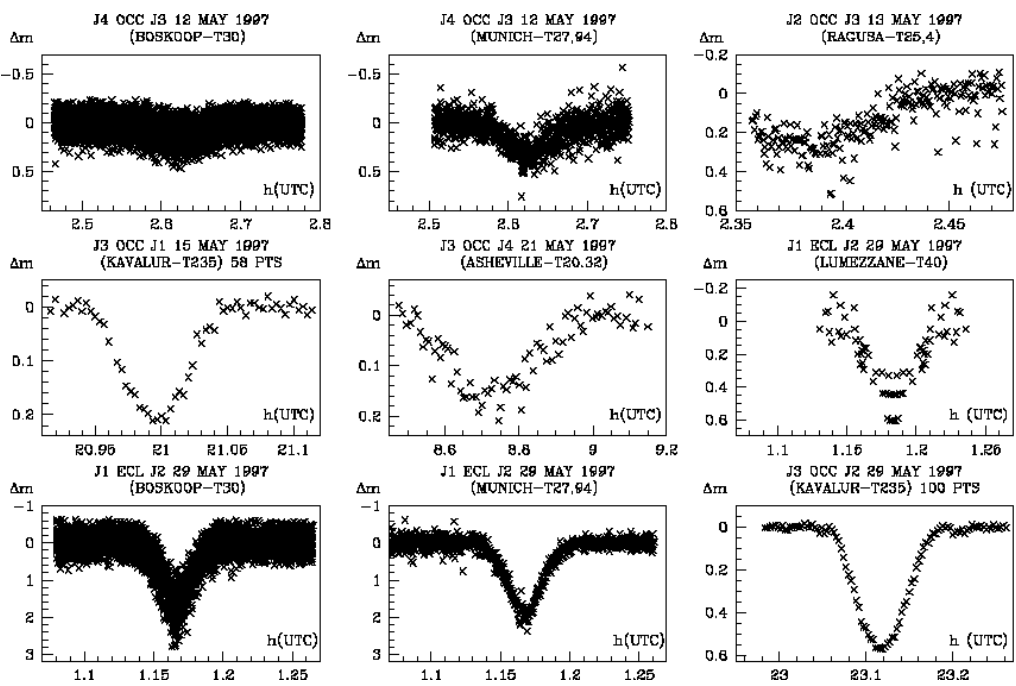

Fig. 2. Lightcurves from the observations of the mutual events of the Galilean satellites in 1996-1997.
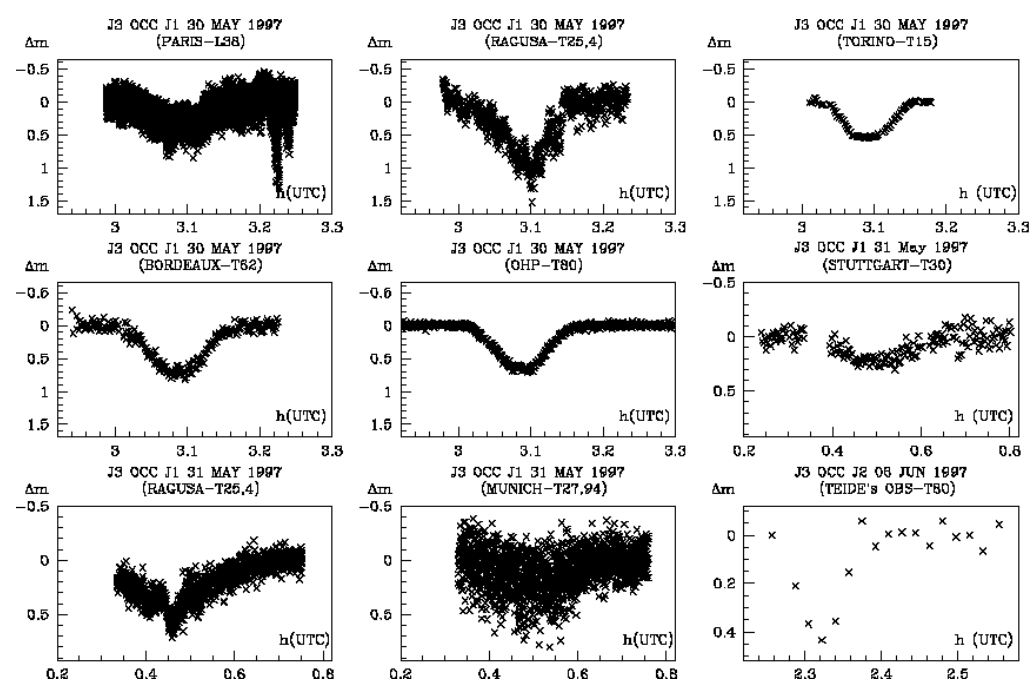

Fig. 3. Lightcurves from the observations of the mutual events of the Galilean satellites in 1996-1997. 
J.-E. Arlot et al.: The PHEMU97 Campaign, Online Material p 14
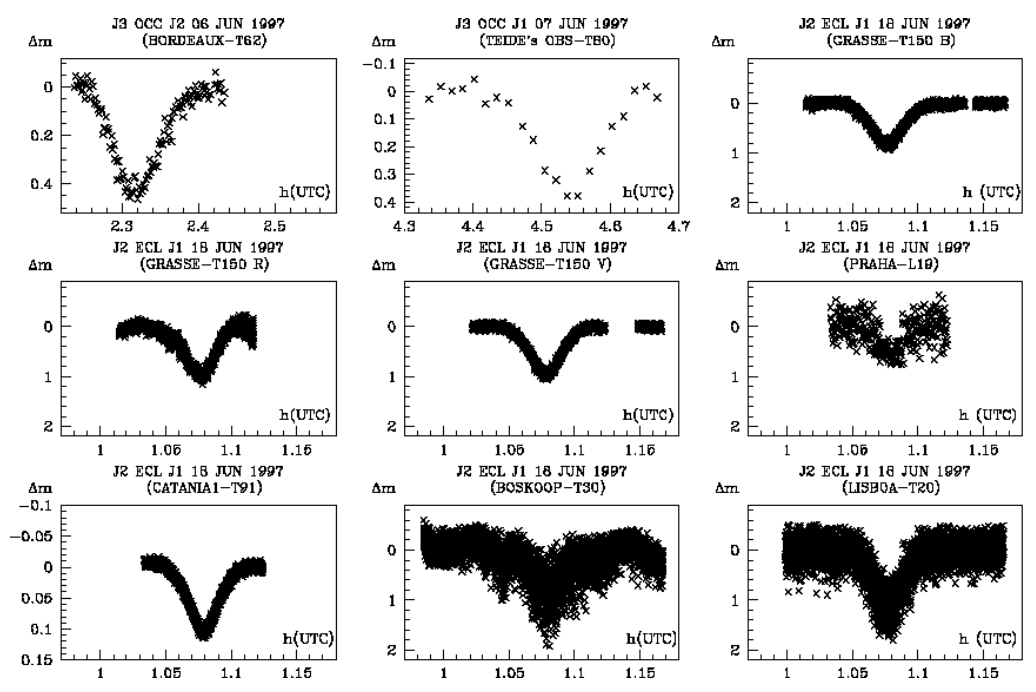

Fig. 4. Lightcurves from the observations of the mutual events of the Galilean satellites in 1996-1997.
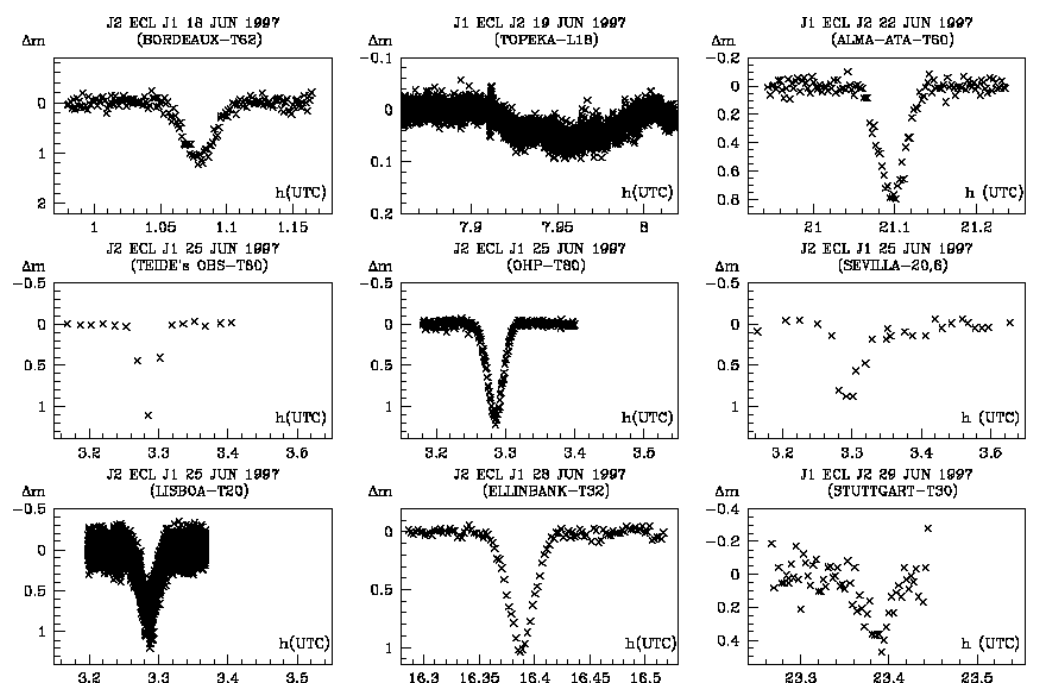

Fig. 5. Lightcurves from the observations of the mutual events of the Galilean satellites in 1996-1997.
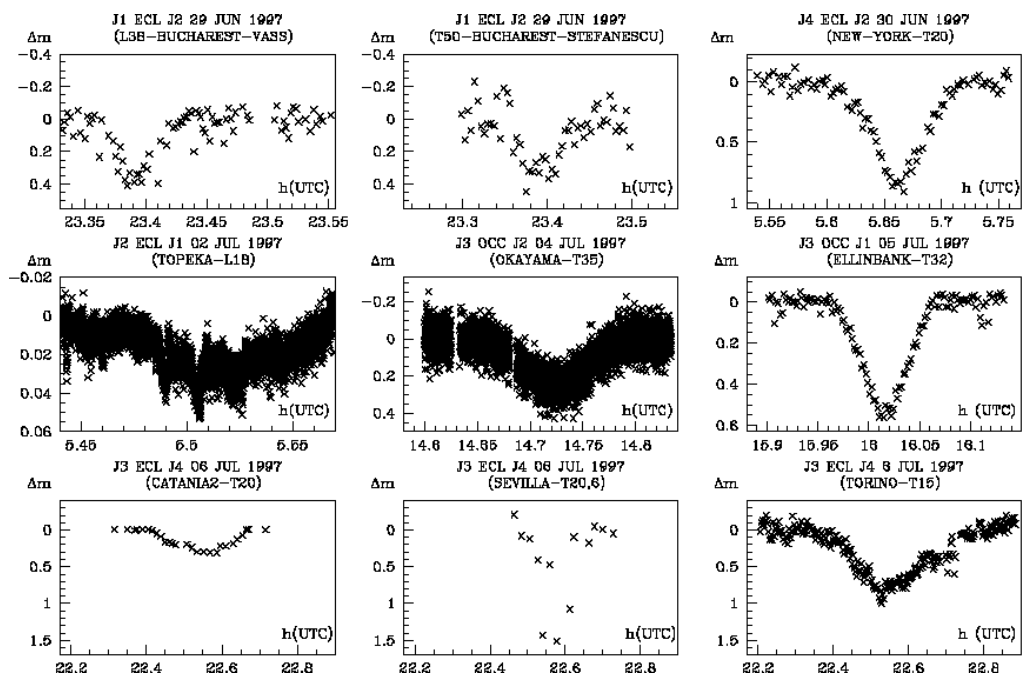

Fig. 6. Lightcurves from the observations of the mutual events of the Galilean satellites in 1996-1997. 
J.-E. Arlot et al.: The PHEMU97 Campaign, Online Material p 15
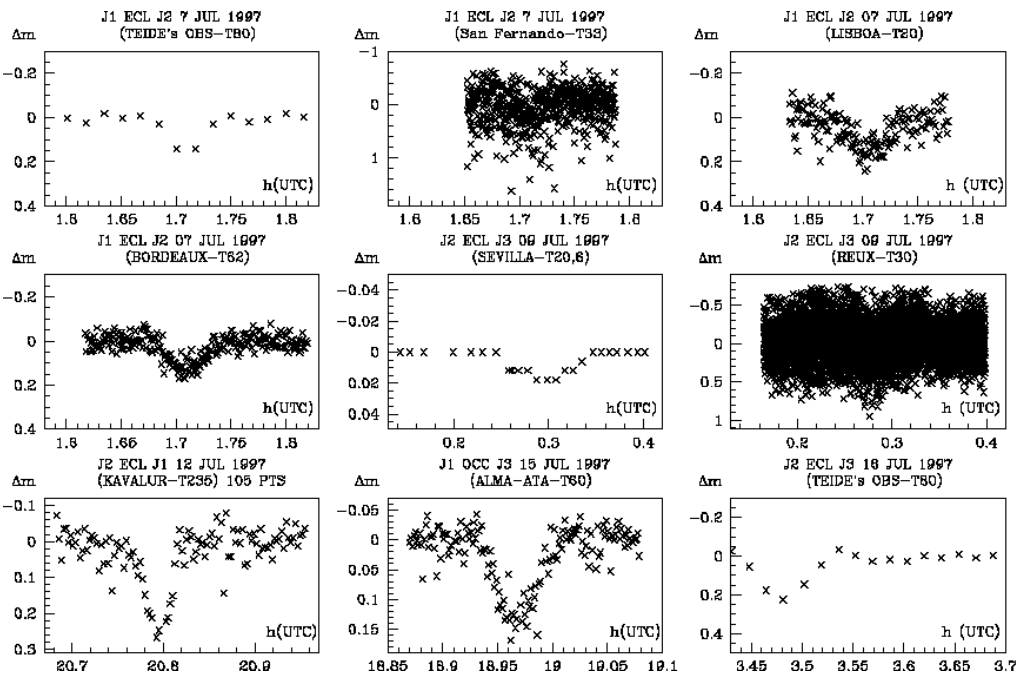

Fig. 7. Lightcurves from the observations of the mutual events of the Galilean satellites in 1996-1997.
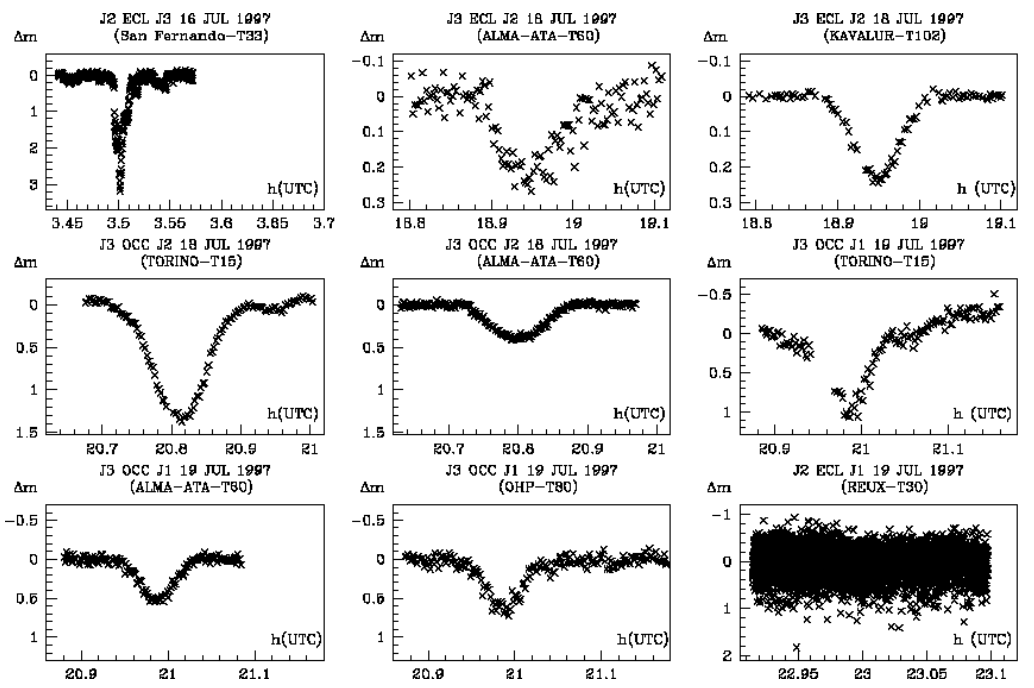

Fig. 8. Lightcurves from the observations of the mutual events of the Galilean satellites in 1996-1997.
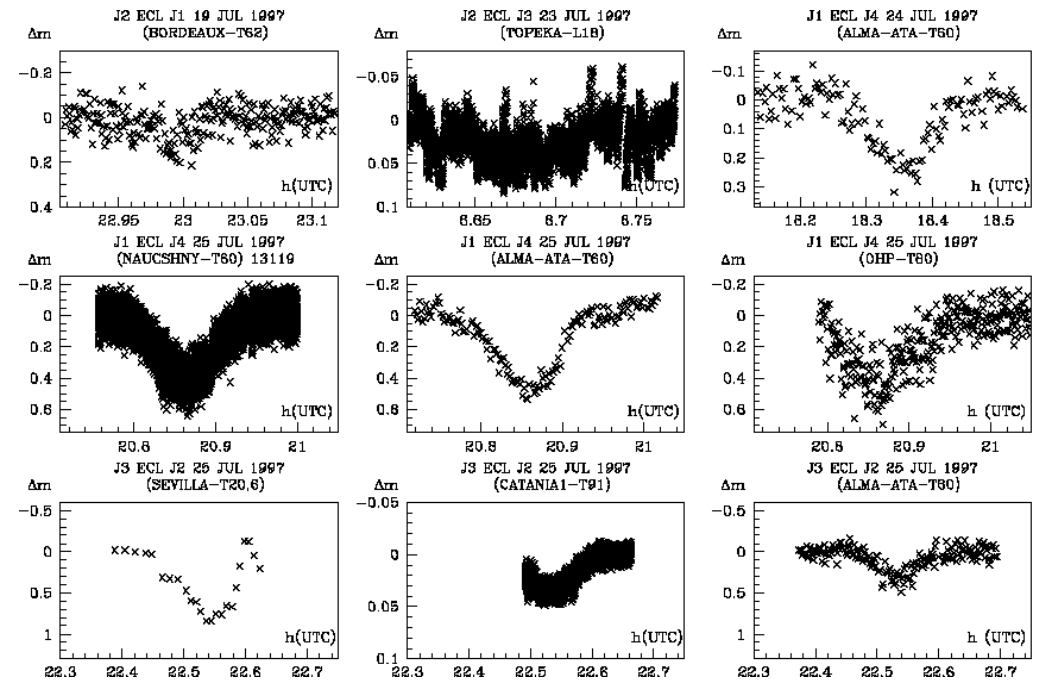

Fig. 9. Lightcurves from the observations of the mutual events of the Galilean satellites in 1996-1997. 
J.-E. Arlot et al.: The PHEMU97 Campaign, Online Material p 16
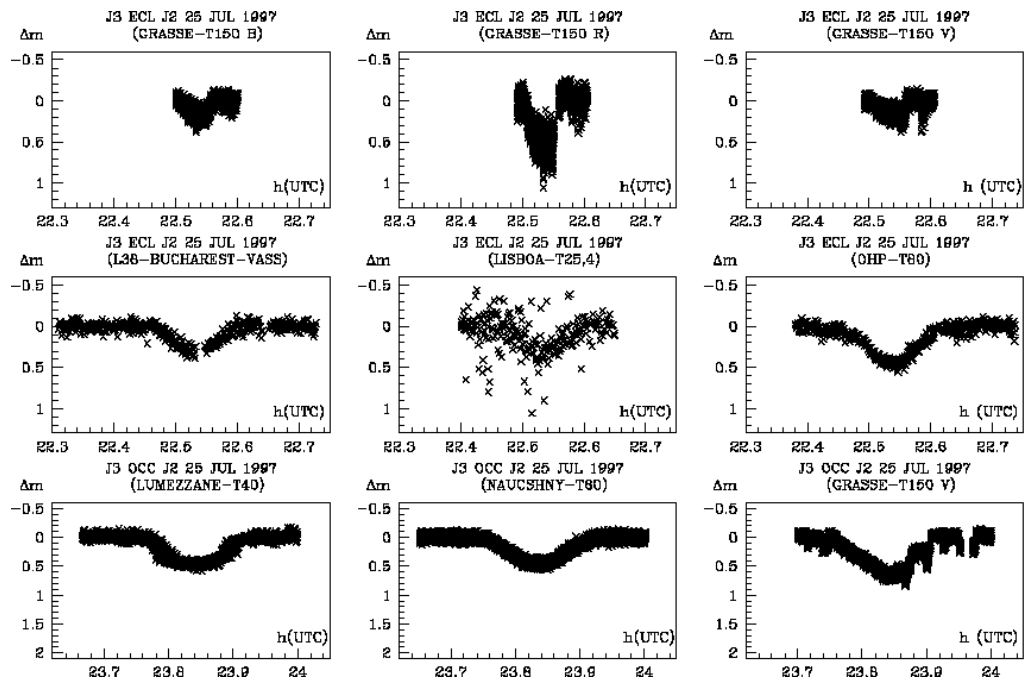

Fig. 10. Lightcurves from the observations of the mutual events of the Galilean satellites in 1996-1997.
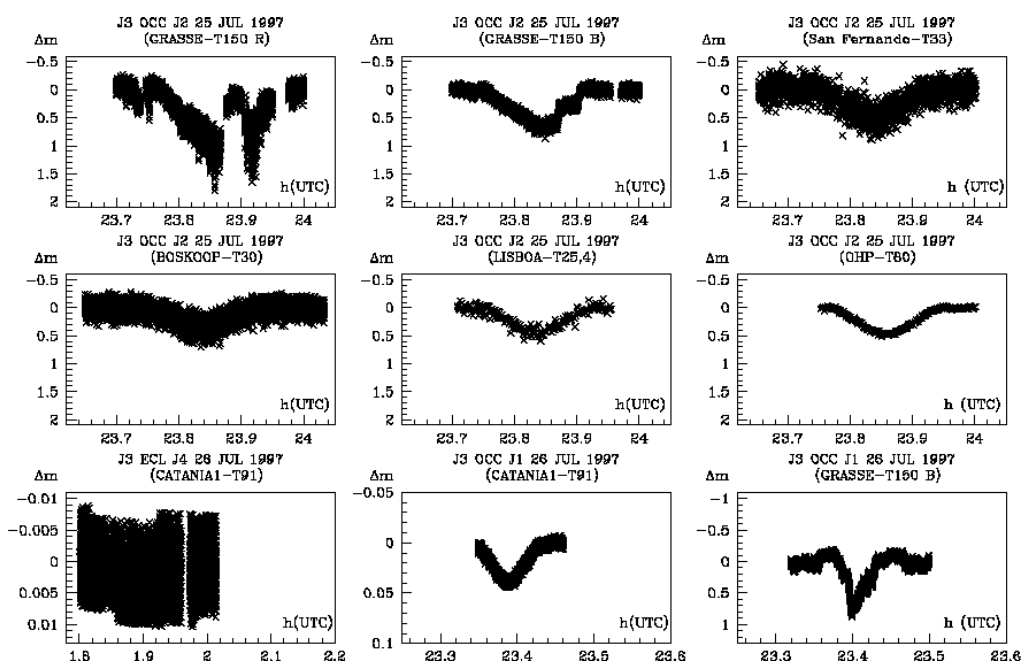

Fig. 11. Lightcurves from the observations of the mutual events of the Galilean satellites in 1996-1997.
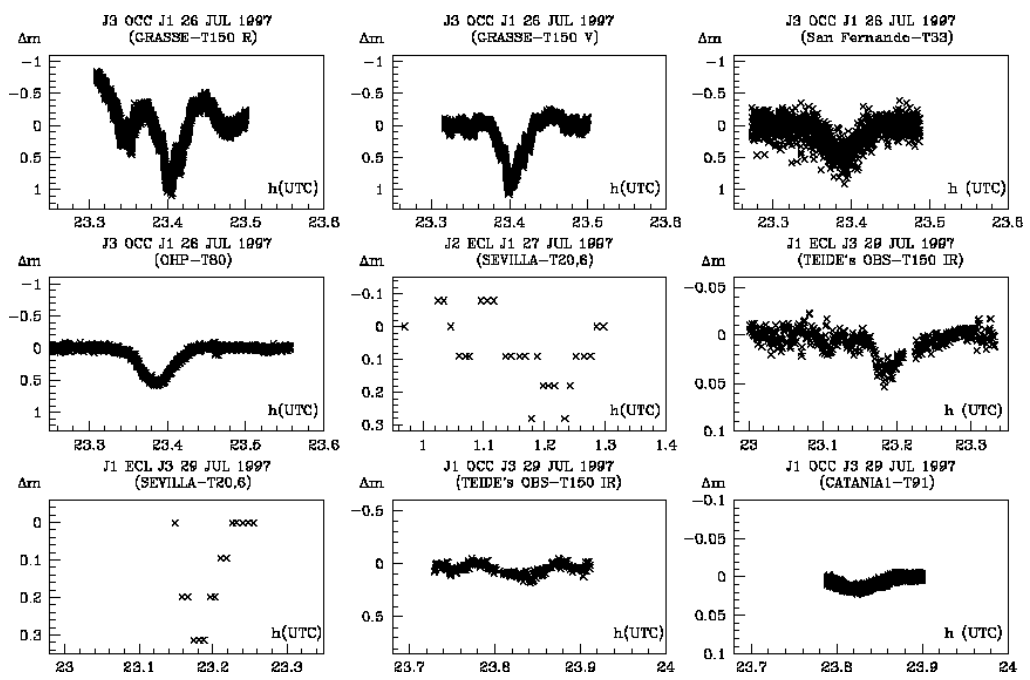

Fig. 12. Lightcurves from the observations of the mutual events of the Galilean satellites in 1996-1997. 
J.-E. Arlot et al.: The PHEMU97 Campaign, Online Material p 17
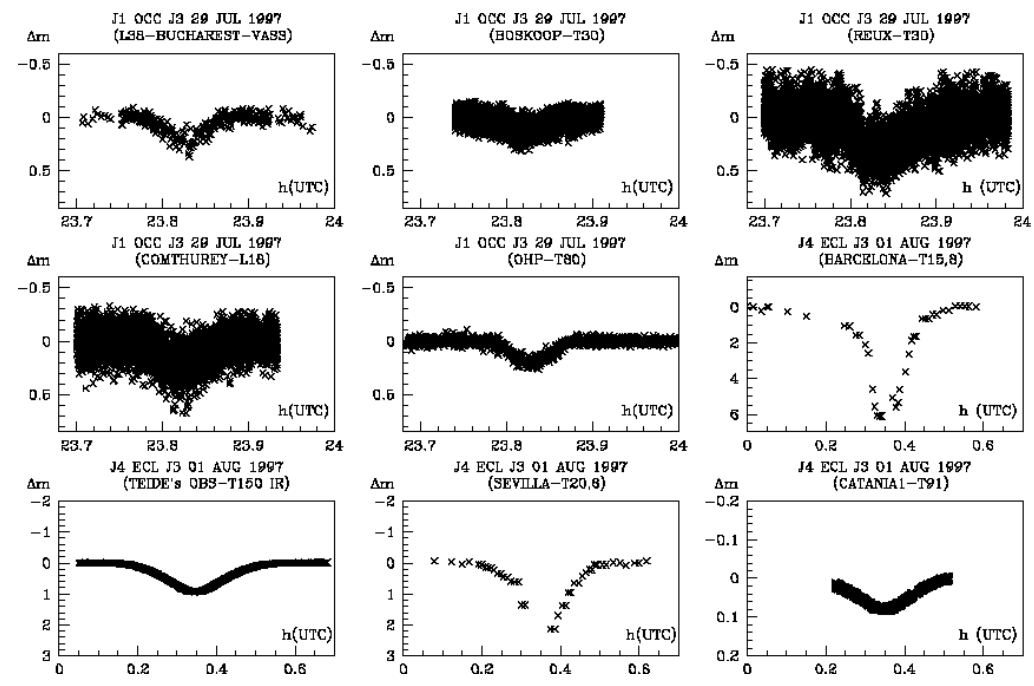

Fig. 13. Lightcurves from the observations of the mutual events of the Galilean satellites in 1996-1997.
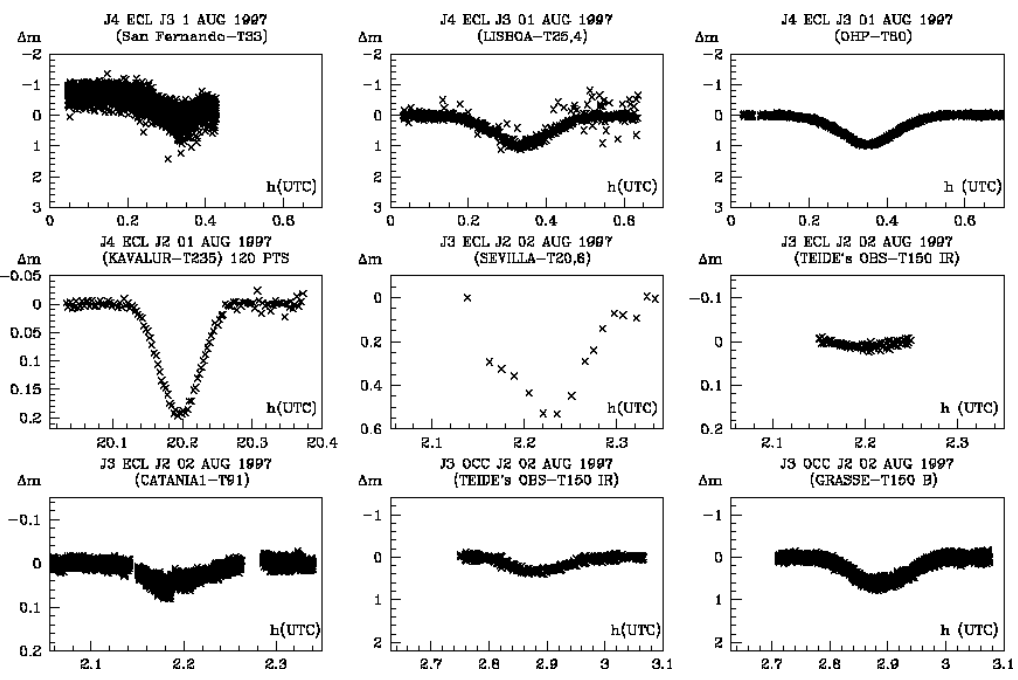

Fig. 14. Lightcurves from the observations of the mutual events of the Galilean satellites in 1996-1997.
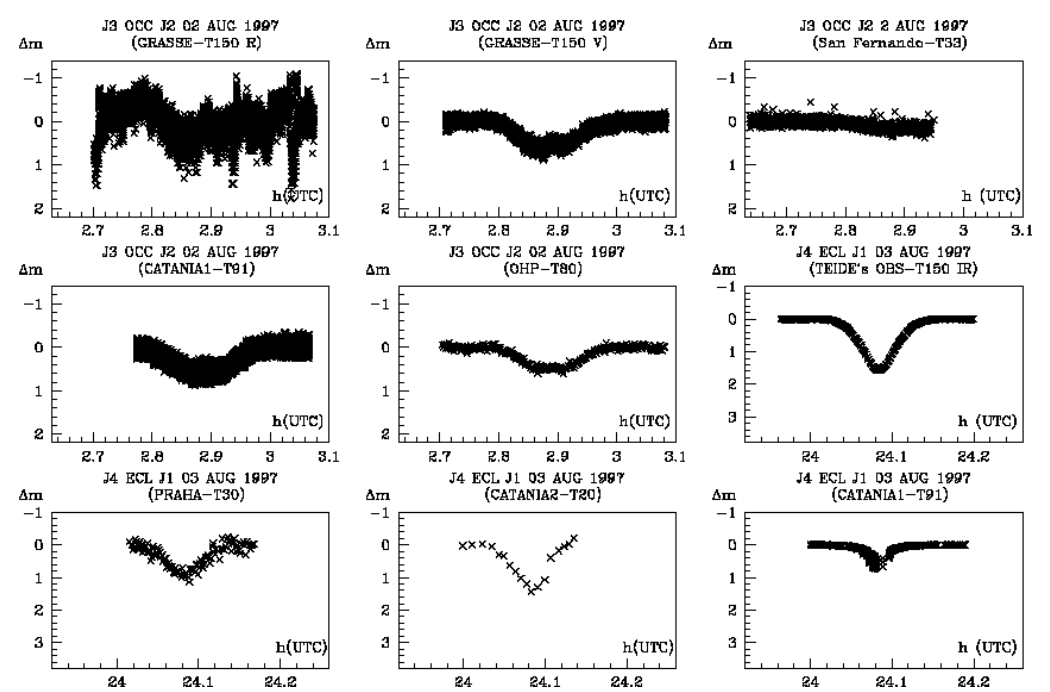

Fig. 15. Lightcurves from the observations of the mutual events of the Galilean satellites in 1996-1997. 
J.-E. Arlot et al.: The PHEMU97 Campaign, Online Material p 18
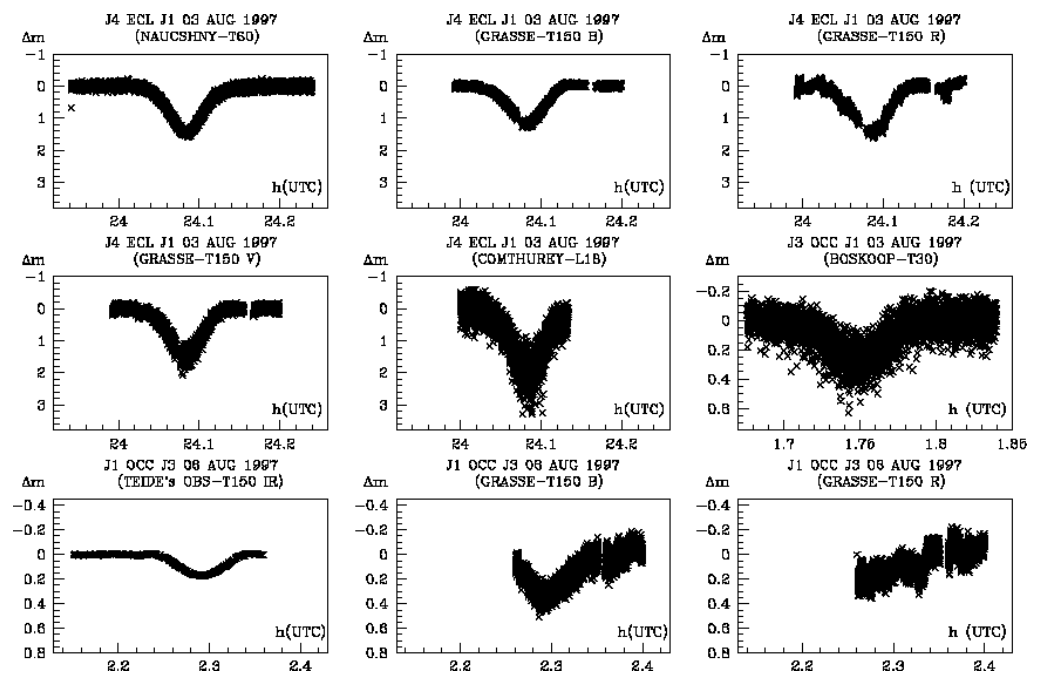

Fig. 16. Lightcurves from the observations of the mutual events of the Galilean satellites in 1996-1997.
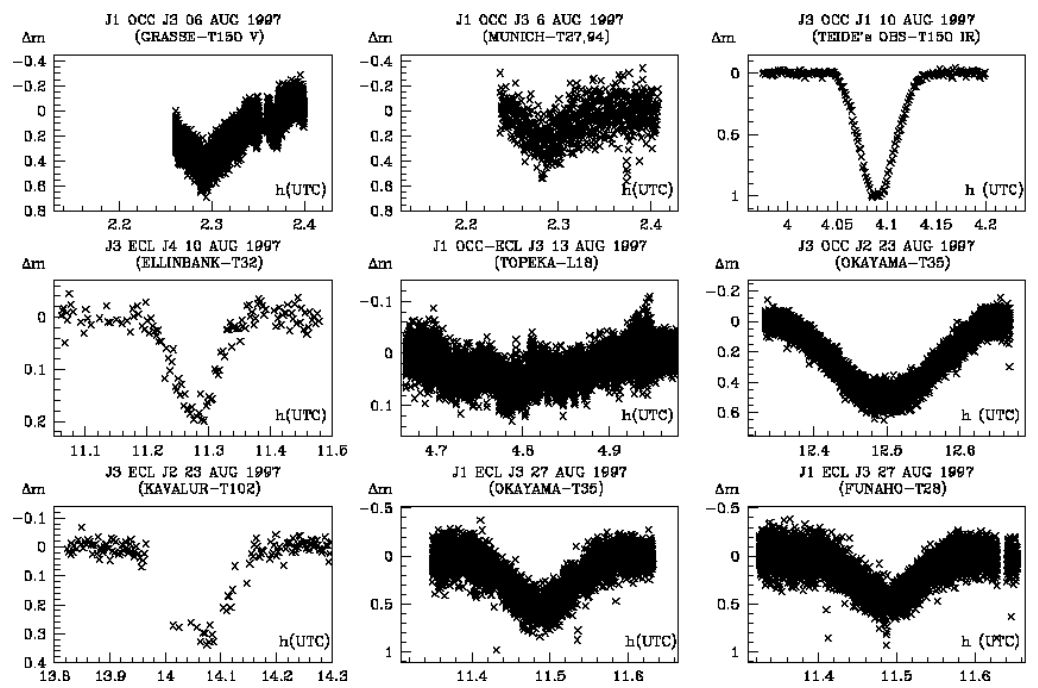

Fig. 17. Lightcurves from the observations of the mutual events of the Galilean satellites in 1996-1997.
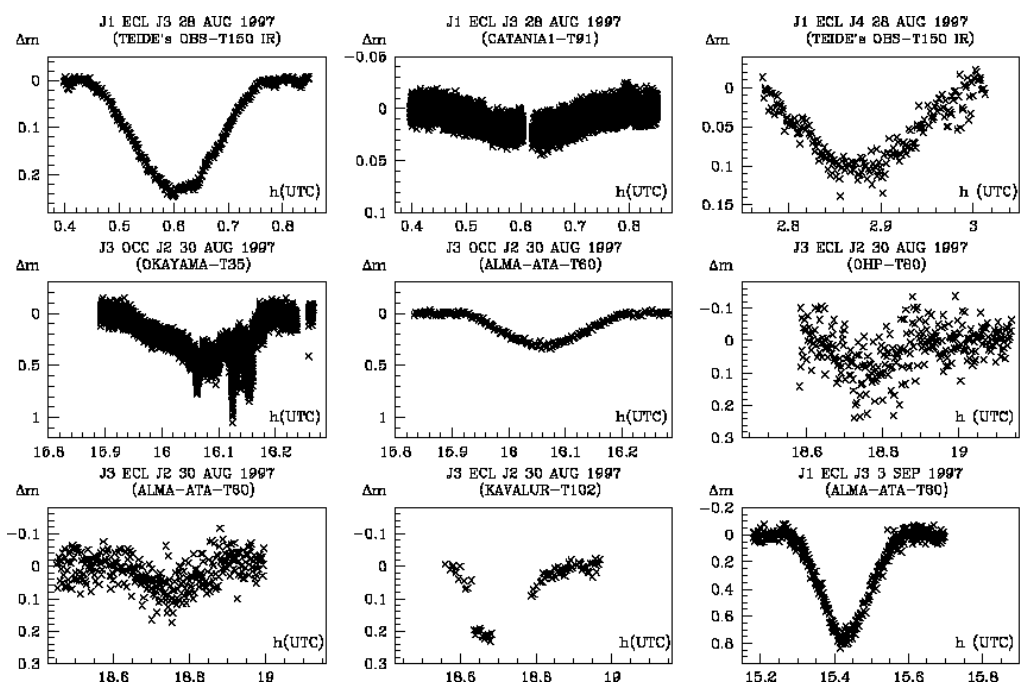

Fig. 18. Lightcurves from the observations of the mutual events of the Galilean satellites in 1996-1997. 
J.-E. Arlot et al.: The PHEMU97 Campaign, Online Material p 19
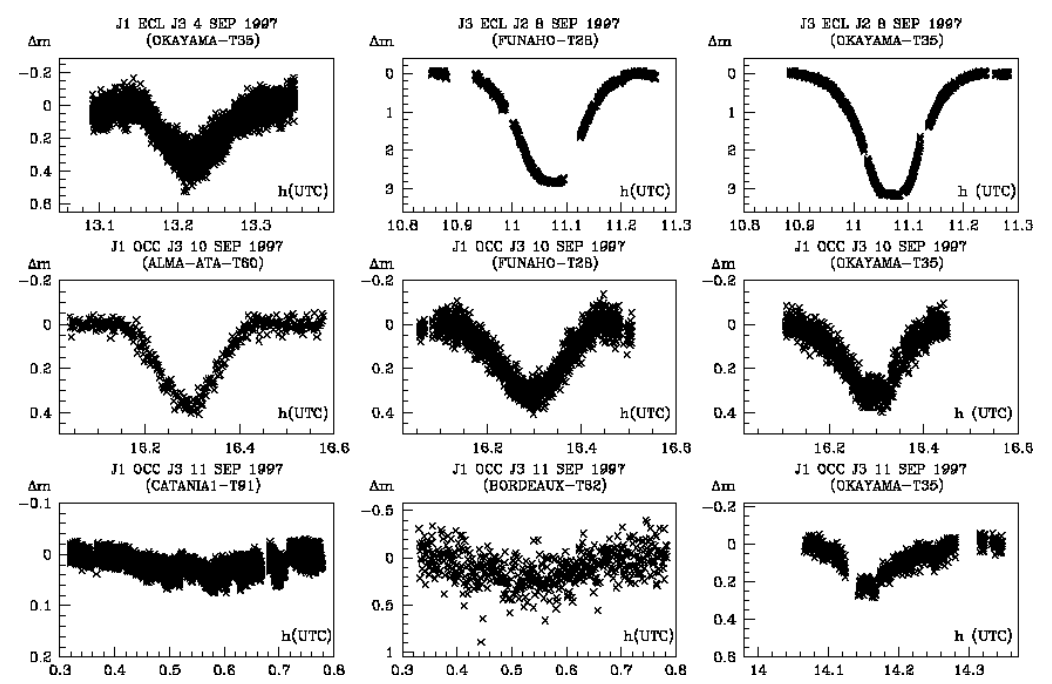

Fig. 19. Lightcurves from the observations of the mutual events of the Galilean satellites in 1996-1997.
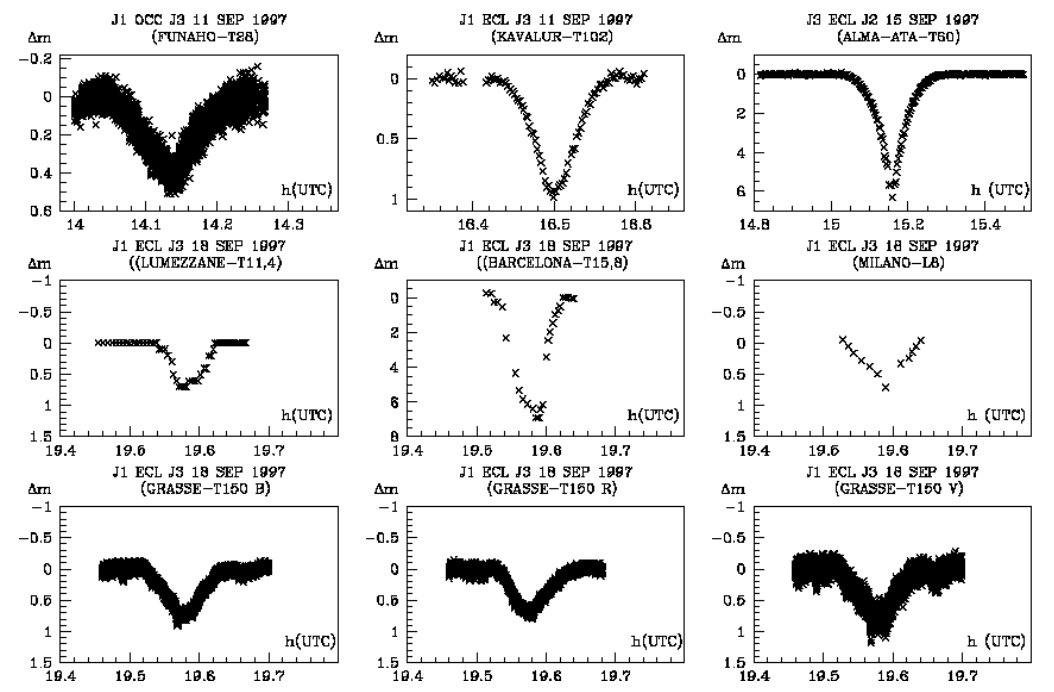

Fig. 20. Lightcurves from the observations of the mutual events of the Galilean satellites in 1996-1997.
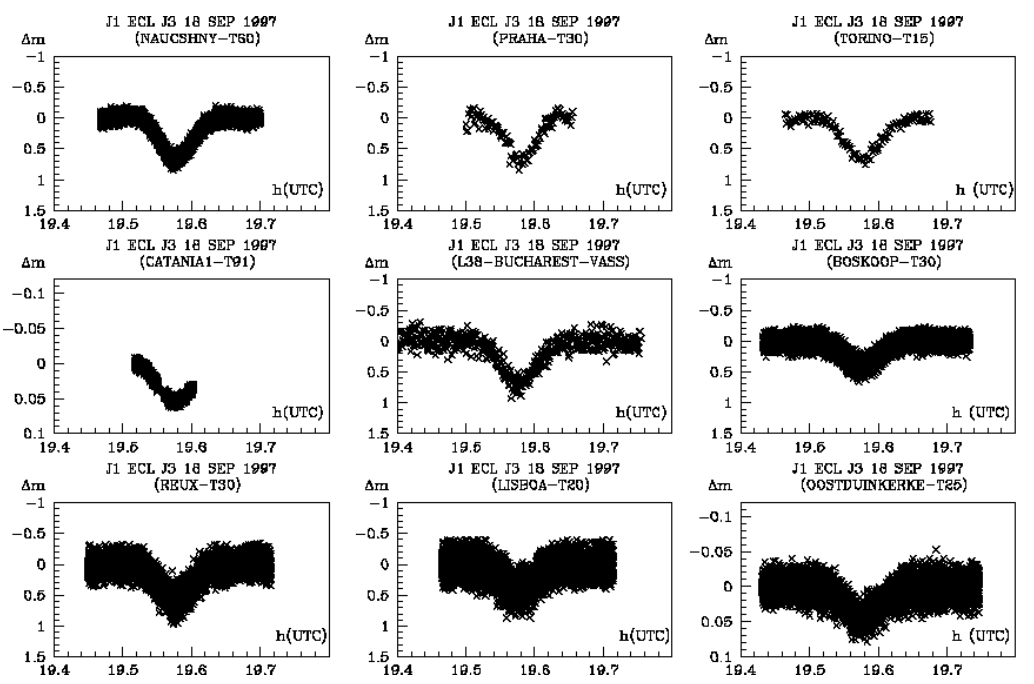

Fig. 21. Lightcurves from the observations of the mutual events of the Galilean satellites in 1996-1997. 
J.-E. Arlot et al.: The PHEMU97 Campaign, Online Material p 20
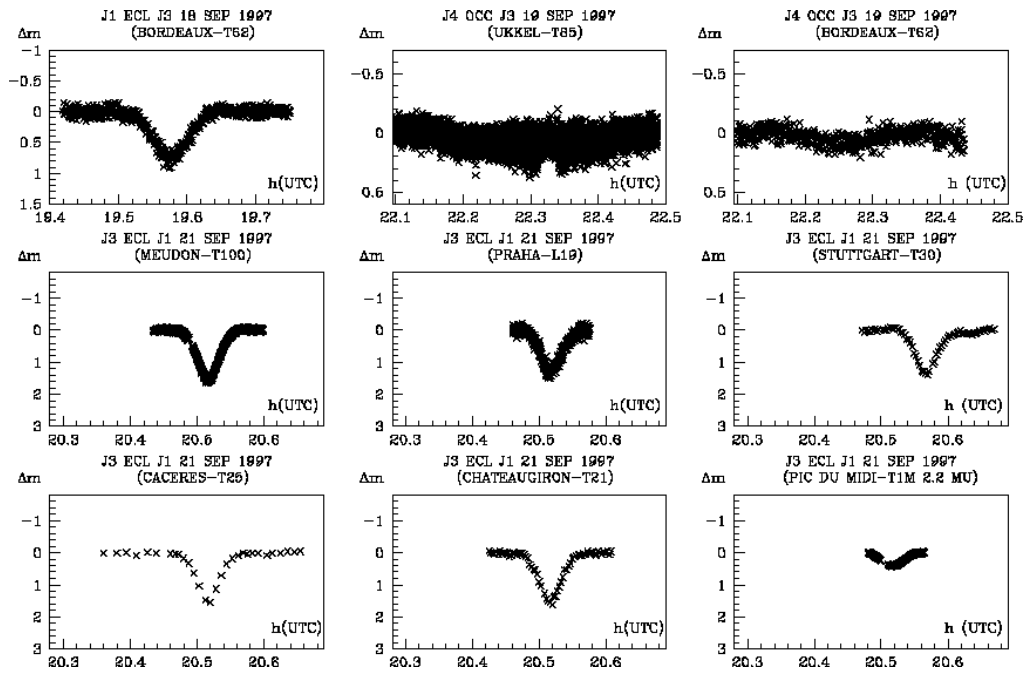

Fig. 22. Lightcurves from the observations of the mutual events of the Galilean satellites in 1996-1997.
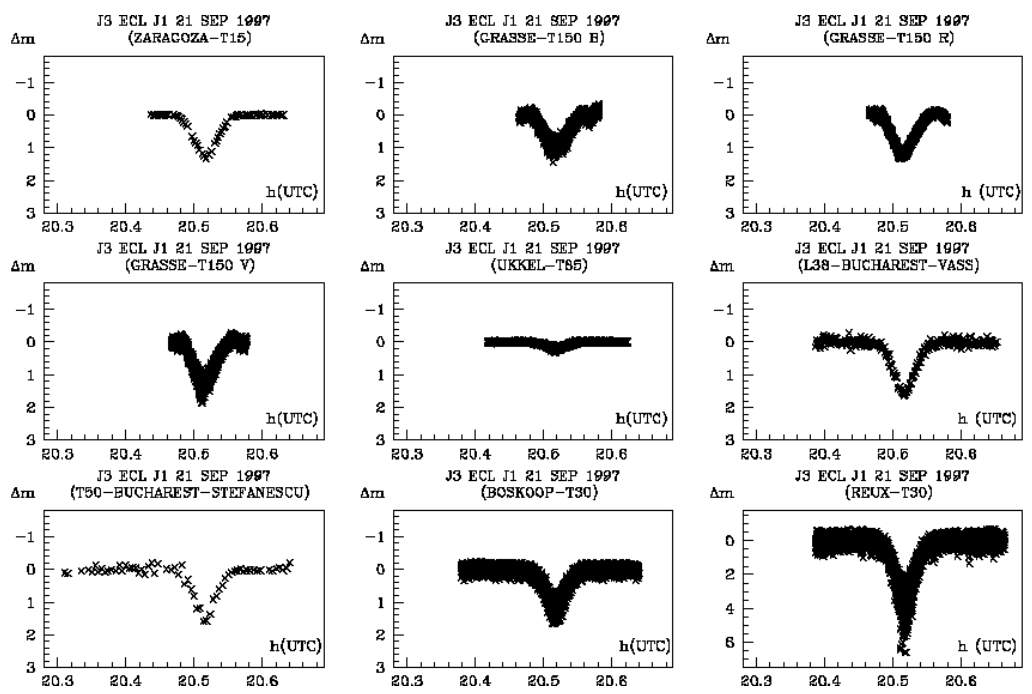

Fig. 23. Lightcurves from the observations of the mutual events of the Galilean satellites in 1996-1997.
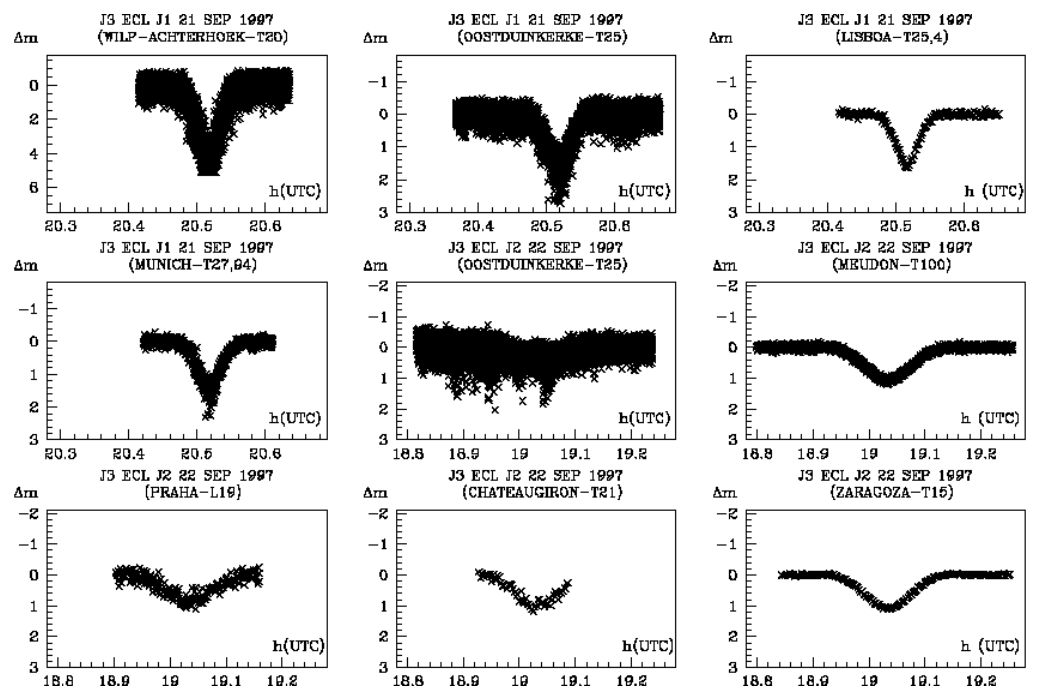

Fig. 24. Lightcurves from the observations of the mutual events of the Galilean satellites in 1996-1997. 
J.-E. Arlot et al.: The PHEMU97 Campaign, Online Material p 21
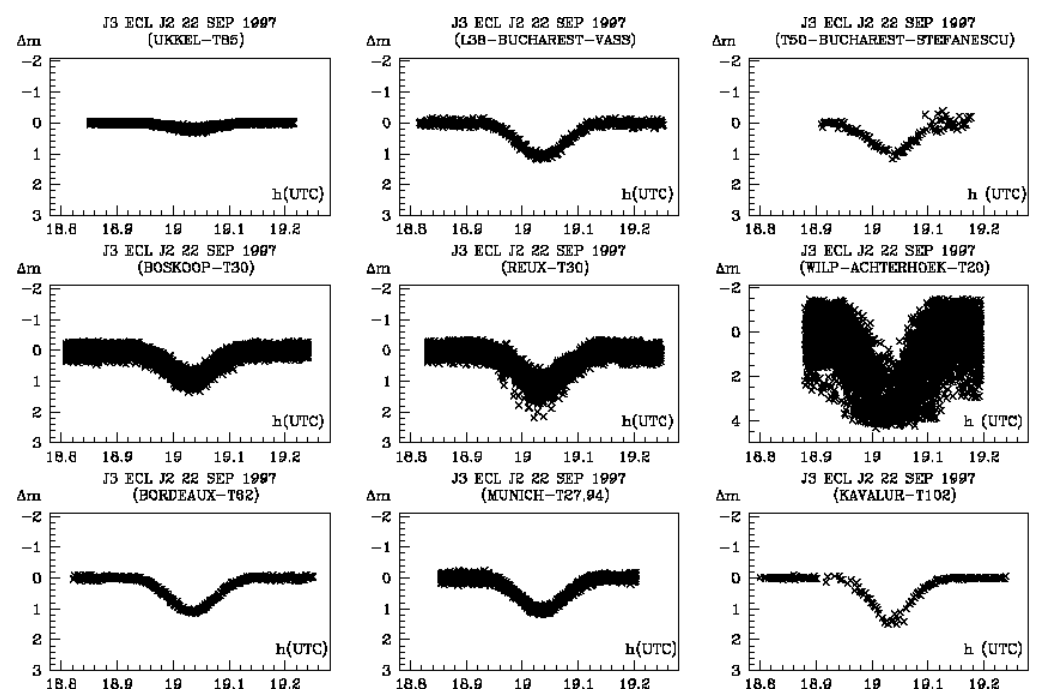

Fig. 25. Lightcurves from the observations of the mutual events of the Galilean satellites in 1996-1997.
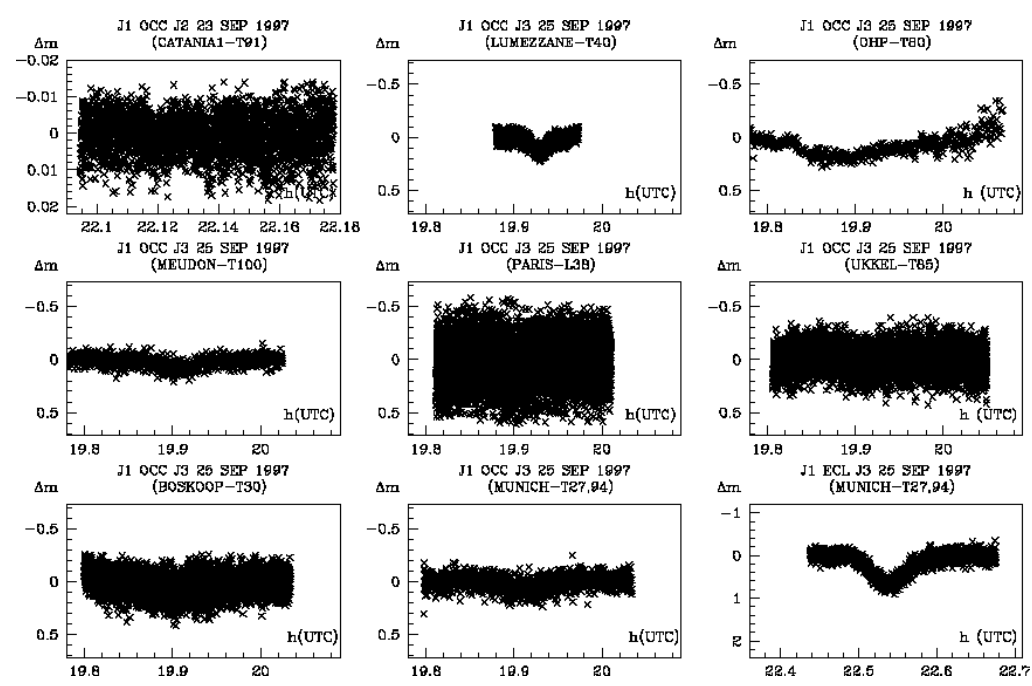

Fig. 26. Lightcurves from the observations of the mutual events of the Galilean satellites in 1996-1997.
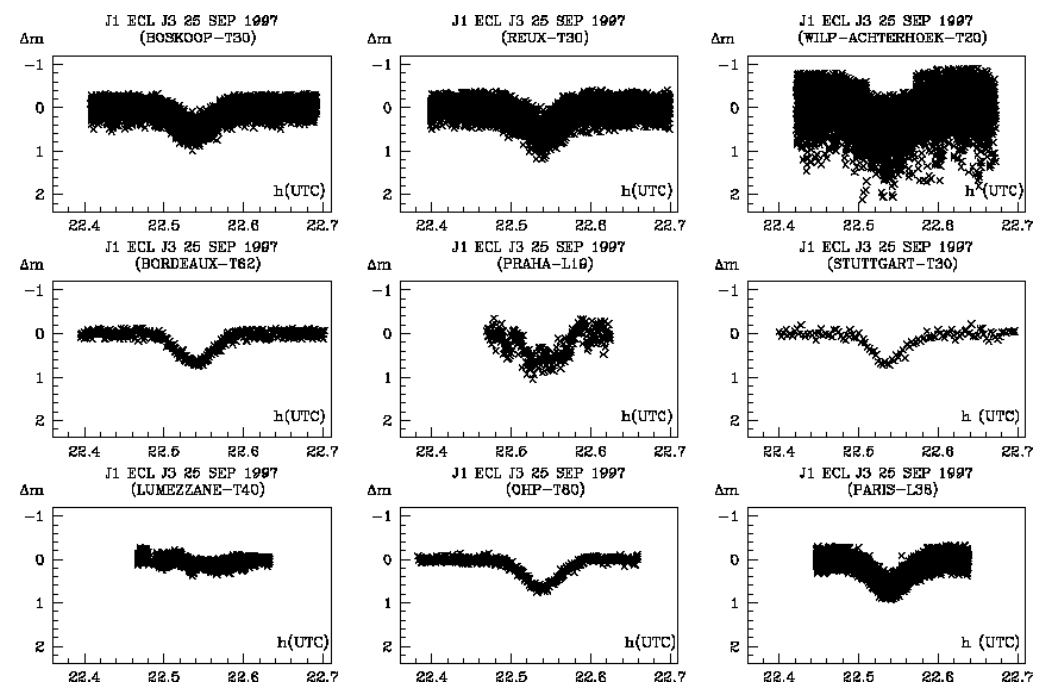

Fig. 27. Lightcurves from the observations of the mutual events of the Galilean satellites in 1996-1997. 
J.-E. Arlot et al.: The PHEMU97 Campaign, Online Material p 22
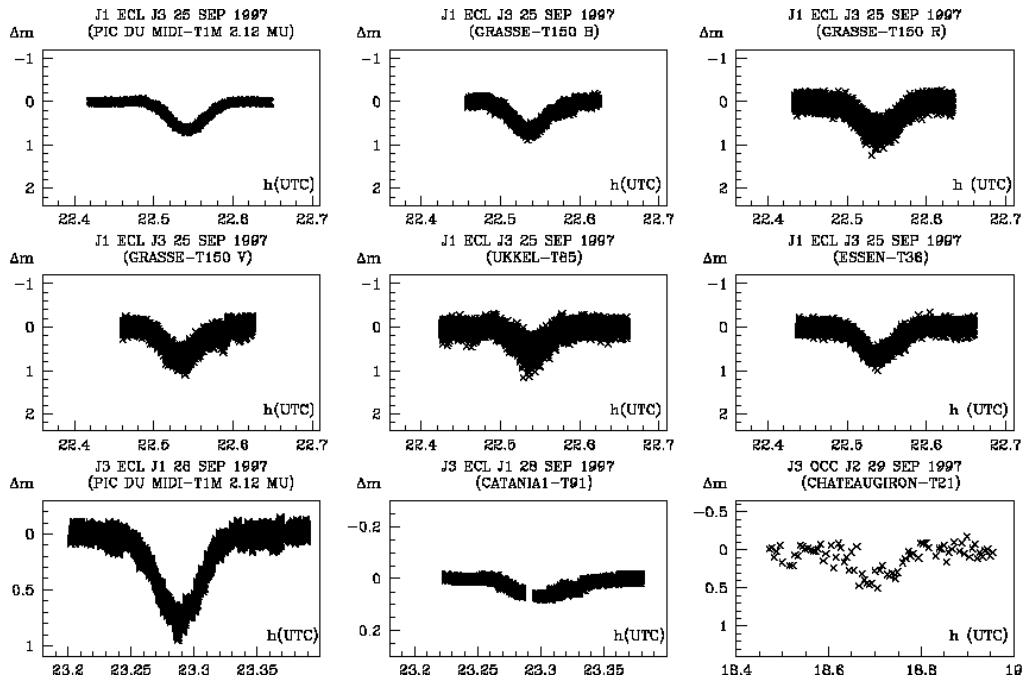

Fig. 28. Lightcurves from the observations of the mutual events of the Galilean satellites in 1996-1997.
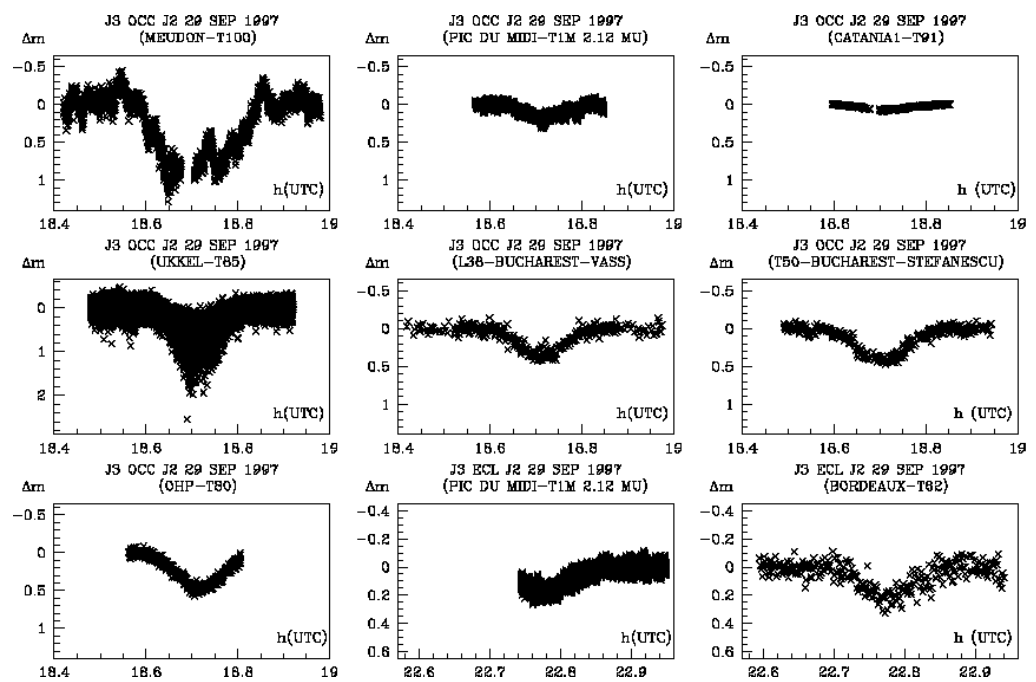

Fig. 29. Lightcurves from the observations of the mutual events of the Galilean satellites in 1996-1997.
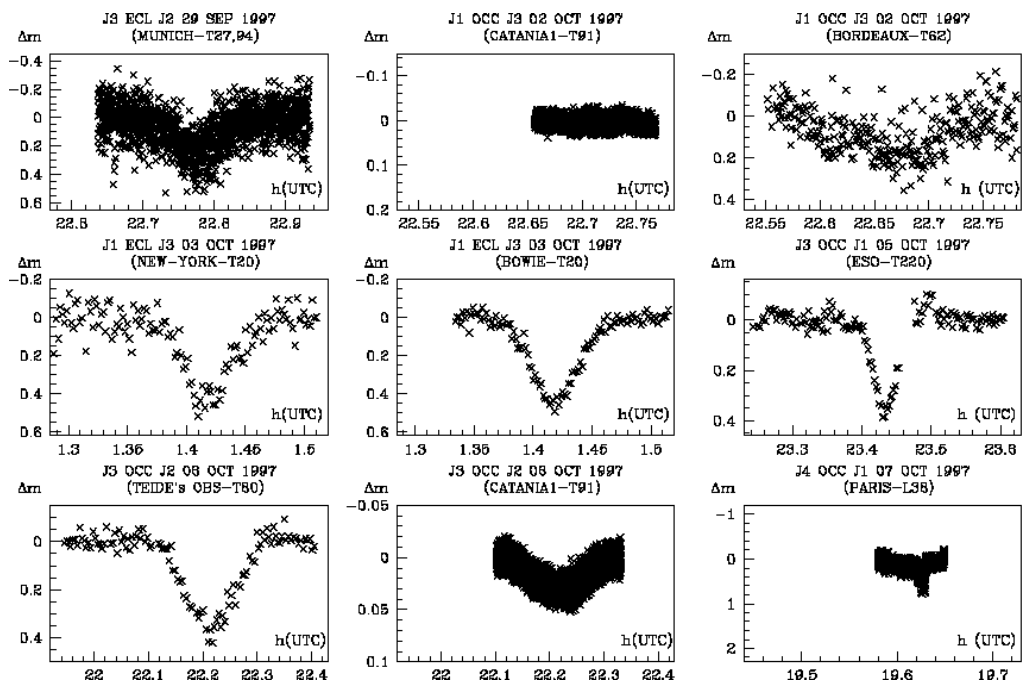

Fig. 30. Lightcurves from the observations of the mutual events of the Galilean satellites in 1996-1997. 
J.-E. Arlot et al.: The PHEMU97 Campaign, Online Material p 23
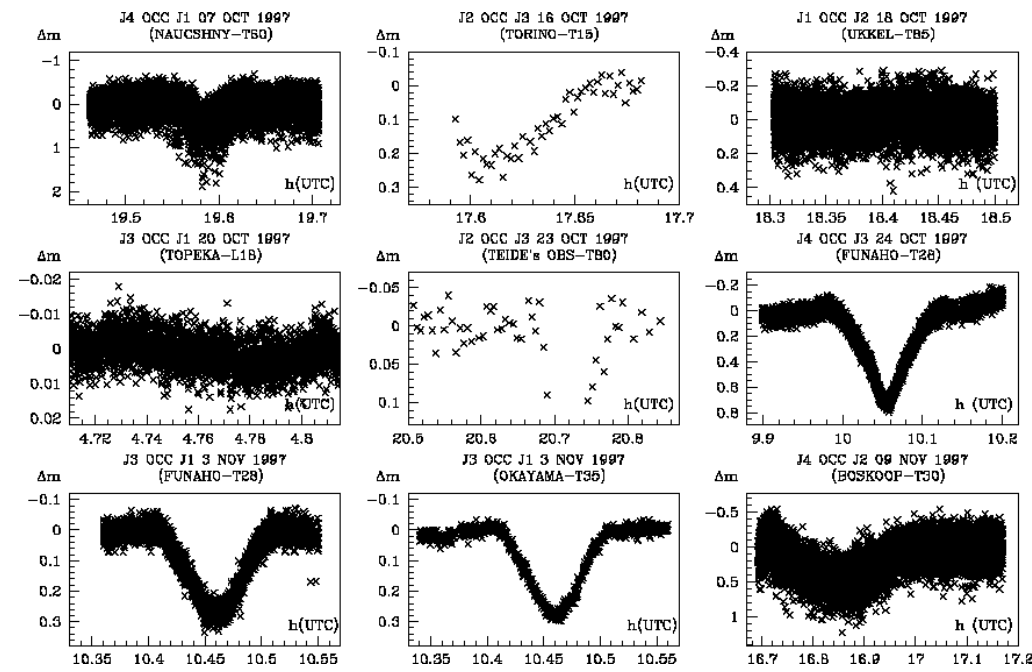

Fig. 31. Lightcurves from the observations of the mutual events of the Galilean satellites in 1996-1997.
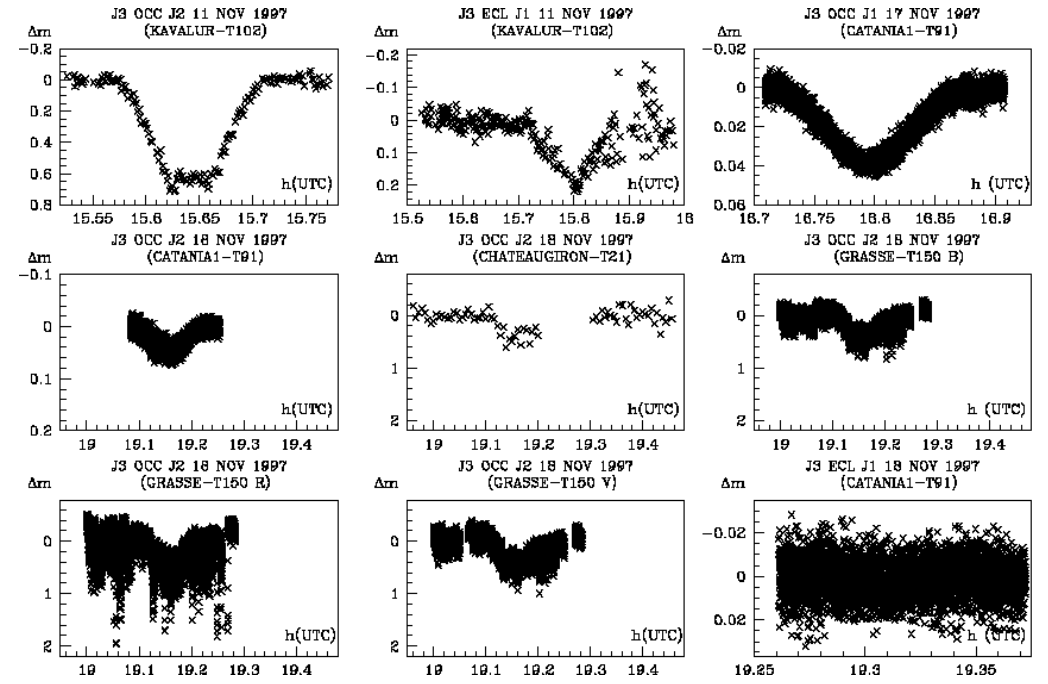

Fig. 32. Lightcurves from the observations of the mutual events of the Galilean satellites in 1996-1997.
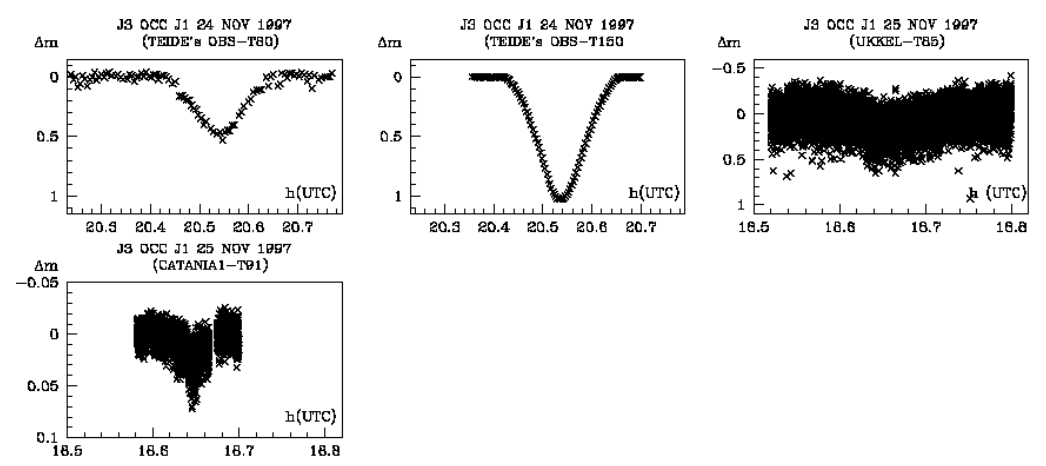

Fig. 33. Lightcurves from the observations of the mutual events of the Galilean satellites in 1996-1997. 